\title{
Partial regularity for mass-minimizing currents in Hilbert spaces
}

\author{
Ambrosio, Luigi ; De Lellis, Camillo ; Schmidt, Thomas
}

\begin{abstract}
Recently, the theory of currents and the existence theory for Plateau's problem have been extended to the case of finite-dimensional currents in infinite-dimensional manifolds or even metric spaces; see [Acta Math. 185 (2000), 1-80] (and also [Proc. Lond. Math. Soc. (3) 106 (2013), 1121-1142], [Adv. Calc. Var. 7 (2014), 227-240] for the most recent developments). In this paper, in the case when the ambient space is Hilbert, we provide the first partial regularity result, in a dense open set of the support, for n-dimensional integral currents which locally minimize the mass. Our proof follows with minor variants [Indiana Univ. Math. J. 31 (1982), 415-434], implementing Lipschitz approximation and harmonic approximation without indirect arguments and with estimates which depend only on the dimension $\mathrm{n}$ and not on codimension or dimension of the target space.
\end{abstract}

DOI: https://doi.org/10.1515/crelle-2015-0011

Posted at the Zurich Open Repository and Archive, University of Zurich

ZORA URL: https://doi.org/10.5167/uzh-111059

Journal Article

Accepted Version

Originally published at:

Ambrosio, Luigi; De Lellis, Camillo; Schmidt, Thomas (2018). Partial regularity for mass-minimizing currents in Hilbert spaces. Journal für die Reine und Angewandte Mathematik, 2018(734):99-144.

DOI: https://doi.org/10.1515/crelle-2015-0011 


\title{
PARTIAL REGULARITY FOR MASS-MINIMIZING CURRENTS IN HILBERT SPACES
}

\author{
LUIGI AMBROSIO, CAMILLO DE LELLIS, AND THOMAS SCHMIDT
}

\begin{abstract}
Recently, the theory of currents and the existence theory for Plateau's problem have been extended to the case of finite-dimensional currents in infinitedimensional manifolds or even metric spaces; see [5] (and also [7, 37] for the most recent developments). In this paper, in the case when the ambient space is Hilbert, we provide the first partial regularity result, in a dense open set of the support, for $n$-dimensional integral currents which locally minimize the mass. Our proof follows with minor variants [32], implementing Lipschitz approximation and harmonic approximation without indirect arguments and with estimates which depend only on the dimension $n$ and not on codimension or dimension of the target space.
\end{abstract}

\section{Contents}

1. Introduction 1

2. Notation and preliminaries 4

3. Codimension-free elliptic estimates $\quad 12$

4. The $\varepsilon$-regularity theorem 16

5. Vertical separation and Lipschitz approximation 17

6. Comparison with harmonic functions and excess decay 24

7. Partial regularity 39

Appendix A. Cartesian products and homotopy construction 41

Appendix B. Monotonicity formula and density results $\quad 45$

References $\quad 47$

\section{INTRODUCTION}

In recent years the theory of currents has undergone several developments, finding suitable extensions to metric spaces [5, 29] and general group coefficients [39, 38, 16]. These ideas have led to general existence results for Plateau's problem [5, 36, 7, 37] which cover also non-smooth and infinite-dimensional spaces, and the relevant

Date: March 4, 2013.

2010 Mathematics Subject Classification. Primary 49Q20, 49N60; Secondary 49Q15, 35B65.

The research leading to these results has received funding from the European Research Council under the European Community's Seventh Framework Programme (FP7/2007-2013) / ERC Grant agreement GeMeThnES nº 246923. 
techniques (in particular an intrinsic approach to metric-space valued Sobolev and BV maps [3] and the Jerrard-Soner BV estimate [26, 27]) had also an impact on the classical theory, leading to more powerful Lipschitz approximation results $[14,15]$ and rectifiability criteria [25].

In a similar vein, in this paper we investigate regularity properties of $n$-dimensional mass-minimizing integral currents $T$ in $\mathcal{H}$, where both the finite dimension $n \in \mathbb{N}=$ $\{1,2,3, \ldots\}$ and the infinite-dimensional separable ${ }^{1}$ Hilbert space $\mathcal{H}$ are fixed for all of the following. Particularly, we treat those currents which appear as minimizers of the general Plateau problem (see [7] for the corresponding existence result)

$$
\min \left\{\mathbf{M}(T): T \in \mathbf{I}_{n}(\mathcal{H}), \partial T=S\right\} .
$$

Here, $S \in \mathbf{I}_{n-1}(\mathcal{H})$ is an arbitrary cycle with bounded support, and we have started to use the notations explained in Section 2.

Since our arguments are completely local in nature, we will rather deal with the class of local minimizers, defined as follows (for minimizers in (1.1) one may take $\Omega=\mathcal{H})$ :

Definition 1.1 (locally mass-minimizing currents). We call $T \in \mathbf{I}_{n}(\mathcal{H})$ locally massminimizing in an open set $\Omega \subset \mathcal{H}$ if there holds

$$
\mathbf{M}(T) \leq \mathbf{M}(T+R) \quad \text { whenever } \quad R \in \mathbf{I}_{n}(\mathcal{H}), \partial R=0, \operatorname{dist}(\operatorname{spt} R, \mathcal{H} \backslash \Omega)>0 .
$$

As in the finite-dimensional theory, we call regular set $\operatorname{Reg} T$ of $T \in \mathbf{I}_{n}(\mathcal{H})$ the collection of all $z \in \operatorname{spt} T$ such that $T$ is represented near $z$ by a $\mathrm{C}^{1}$ graph with some multiplicity $m$ : more precisely, it is the set of all $z \in \operatorname{spt} T$ such that

$$
T\left\llcorner\left[z+\mathbf{B}_{\varrho}^{\pi}+\mathbf{B}_{\varrho}^{\perp \pi}\right]=m \llbracket \text { Graph } f \rrbracket\right.
$$

holds for some $\varrho>0$, some $n$-plane $\pi$ in $\mathcal{H}$, some non-zero integer $m$, and some $\mathrm{C}^{1}$-function $f: \mathrm{p}^{\pi}(z)+\mathbf{B}_{\varrho}^{\pi} \rightarrow(\operatorname{Span} \pi)^{\perp}$ (we refer once more to Section 2 for notation and precise definitions).

Evidently, $\operatorname{Reg} T$ is always an open subset of spt $T \backslash$ spt $\partial T$. Providing the first partial regularity result for mass-minimizing currents in infinite dimensions, we will however show that, for minimizers in (1.1), $\operatorname{Reg} T$ is also dense in spt $T \backslash \operatorname{spt} \partial T$. This assertion follows in fact from the choice $\Omega=\mathcal{H} \backslash$ spt $\partial T$ in our following main theorem:

Theorem 1.2 (partial regularity for locally mass-minimizing currents). If $T \in \mathbf{I}_{n}(\mathcal{H})$ is locally mass-minimizing in an open set $\Omega \subset \mathcal{H}$ with $(\partial T)\llcorner\Omega \equiv 0$, then $\operatorname{Reg} T$ is dense in $\Omega \cap \operatorname{spt} T$.

To put our result in perspective, let us mention that in the case $n=1$ one can represent $T$ in $\Omega$ as a locally finite union of non-intersecting line segments (endowed with orientations and multiplicities), so that we have full interior regularity $\Omega \cap \operatorname{spt} T=$

\footnotetext{
${ }^{1}$ We remark that the separability assumption on $\mathcal{H}$ is not restrictive, as the metric currents of [5] are always concentrated on a $\sigma$-compact set and have thus separable support; see [5, Lemma 2.9].
} 
$\Omega \cap \operatorname{Reg} T$ in this situation. Therefore, we focus in the sequel on the (much) more challenging case $n \geq 2$.

In the finite-dimensional case $\mathcal{H} \cong \mathbb{R}^{N}$, the regularity theory has been developed first in codimension 1, i.e. for $n=N-1$, and then in general codimension. In codimension 1 (see for instance $[23,34]$ for accounts of the theory), the partial regularity result of [12] and a detailed analysis of singular minimal cones [33, 21] gave the optimal bound $n-7$ for the dimension of the singular set $\Sigma_{\Omega}(T)=\Omega \cap(\operatorname{spt} T \backslash \operatorname{Reg} T)$. In higher codimension, the first partial regularity results have been obtained in $[30,2]$. However, the so-called branching phenomenon provided for many years an obstacle to the development of a sharp theory, until Almgren's monumental work, now published in [1], led to the bound $n-2$ for the dimension of $\Sigma_{\Omega}(T)$, optimal in codimension higher than 1. Recently, Almgren's program has been revisited, simplified, and improved in a series of papers $[14,15,13]$ by the second author and Spadaro. Many parts of this program are by now sufficiently robust to work even in infinite dimensions, but at this moment it is not clear whether the whole program, starting from the theory of Dir-minimizing $Q$-valued maps, can be carried out with constants independent of the codimension.

For these reasons, in the present paper we focus on the more modest goal of proving regularity at $(\|T\|$-almost all) non-branching points; however, as a typical outcome, the general form of our $\varepsilon$-regularity result (see Theorem 4.2) also gives that $\Sigma_{\Omega}(T)$ is even $\|T\|$-negligible if the multiplicity of $T$ equals a constant $\|T\|$-a. e. in $\mathcal{H}$. Our proof follows in large parts the approach of [32], valid even for more general functionals than the mass (compare also $[10,8,17]$ ): the main novelty of [32], in comparison to the older strategy of [2] (which is also adapted in [20]), is that the constants involved in the $\varepsilon$-regularity theorem do not arise by contradiction arguments, and therefore - at least in principle - they can be explicitly computed or estimated. In the case of the mass functional our main contribution is to show that, as a matter of fact, all these constants can be bounded using the dimension $n$ only. This, combined with other codimension-free tools (Kirszbraun and Rademacher theorems, for instance), leads to the result.

Finally, we close the introduction by explaining where the restriction to the mass functional comes from. It would not be too difficult, using the tools developed in [5], to provide an existence theory for the minimization of more general "anisotropic" functionals. On the other hand, the linearization of the functional around a given $n$ plane leads to a suitable elliptic PDE for functions defined on a $n$-dimensional plane in $\mathcal{H}$ with values in its infinite-dimensional orthogonal complement: in other words, this is an infinite system of PDEs. The proof of the $\varepsilon$-regularity result crucially depends on estimates for solutions of such a system, specifically $\mathrm{C}^{1, \alpha}$ estimates in [32] and $\mathrm{W}^{1, p}$ estimates (with $p>2$ sufficiently large) in our proof. In case of the mass functional the linearization is the Laplace equation, leading to appropriate estimates for harmonic functions, which in turn can be proved with (more or less) explicit computations, see 
Section 3. It might be a problem of independent interest to identify a more general class of infinite elliptic systems for which analogous estimates hold.

\section{Notation AND PRELIMINARIES}

2.1. Hilbert space geometry. We denote the inner product of the Hilbert space $\mathcal{H}$ as $\langle\cdot, \cdot\rangle$. Moreover, we write $\Lambda_{n}(\mathcal{H})$ for the space of $n$-vectors ${ }^{2}$ over $\mathcal{H}$, and we notice that $\Lambda_{n}(\mathcal{H})$ carries the induced inner product, characterized by

$$
\left\langle z_{1} \wedge z_{2} \wedge \ldots \wedge z_{n}, \xi_{1} \wedge \xi_{2} \wedge \ldots \wedge \xi_{n}\right\rangle=\operatorname{det}\left(\left\langle z_{i}, \xi_{j}\right\rangle\right)_{i, j=1,2, \ldots, n} \quad \text { for } z_{i}, \xi_{j} \in \mathcal{H} .
$$

In the following we will briefly write $|\cdot|$ for the inner product norm on both $\mathcal{H}$ and $\Lambda_{n}(\mathcal{H})$.

If a simple $n$-vector $\pi$ is unitary with respect to the inner product of $(2.1)$, we will call it an $n$-plane in $\mathcal{H}$. This terminology is motivated by the fact that $\pi$ can be identified with an oriented $n$-dimensional subspace of $\mathcal{H}$; indeed, when we represent $\pi=\bigwedge_{i=1}^{n} \pi_{i}$ with orthonormal vectors $\pi_{1}, \pi_{2}, \ldots, \pi_{n} \in \mathcal{H}$, then we write $\operatorname{Span} \pi$ for the $n$-dimensional subspace of $\mathcal{H}$ which is spanned by $\pi_{1}, \pi_{2}, \ldots, \pi_{n}$, and $\pi$ is one of the two possible constant orientations of $\operatorname{Span} \pi$ (notice that $\operatorname{Span} \pi$ depends only on $\pi$, but not on the choice of the $\pi_{i}$ ). Without further mentioning we will identify $\operatorname{Span} \pi$ with $\mathbb{R}^{n}$ in some regards: for instance, while we write $\mathscr{H}^{n}$ for the $n$-dimensional Hausdorff measure on $\mathcal{H}$, we view its restriction to Span $\pi$ as a Lebesgue measure $\mathscr{L}^{n}$, and we do not clearly distinguish between harmonic functions on (subsets of) $\operatorname{Span} \pi$ and $\mathbb{R}^{n}$. Finally, by (Span $\left.\pi\right)^{\perp}$ we denote the orthogonal complement of $\operatorname{Span} \pi$ in $\mathcal{H}$ which is closed and itself an infinite-dimensional separable Hilbert space.

We will sometimes write $\mathrm{L}^{\pi}$ for the Hilbert space of linear maps $\operatorname{Span} \pi \rightarrow(\operatorname{Span} \pi)^{\perp}$, endowed with the Hilbert-Schmidt product. When $\pi_{1}, \pi_{2}, \ldots, \pi_{n}$ is an orthonormal basis of $\operatorname{Span} \pi$, then this product is given by

$$
\left\langle\ell, \ell_{0}\right\rangle:=\sum_{i=1}^{n}\left\langle\ell \pi_{i}, \ell_{0} \pi_{i}\right\rangle \quad \text { for } \ell, \ell_{0} \in \mathrm{L}^{\pi},
$$

with the inner product of $\mathcal{H}$ on the right hand side (and again the definition does not depend on the choice of the $\left.\pi_{i}\right)$. We also keep writing $|\cdot|$ for the corresponding Hilbert-Schmidt norm, that is

$$
|\ell|:=\sqrt{\sum_{i=1}^{n}\left|\ell \pi_{i}\right|^{2}} \quad \text { for } \ell \in \mathrm{L}^{\pi} .
$$

Furthermore, we define the ball with center 0 and radius $r$ in $\mathcal{H}$ by

$$
\mathbf{B}_{r}:=\{z \in \mathcal{H}:|z|<r\}
$$

\footnotetext{
${ }^{2}$ We adopt the convention that $\Lambda_{n}(\mathcal{H})$ contains only finite sums of simple $n$-vectors; however, this will not play an essential role in the following.
} 
and its counterparts in $\operatorname{Span} \pi$ and $(\operatorname{Span} \pi)^{\perp}$ respectively by

$$
\mathbf{B}_{r}^{\pi}:=\mathbf{B}_{r} \cap \operatorname{Span} \pi \quad \text { and } \quad \mathbf{B}_{r}^{\perp \pi}:=\mathbf{B}_{r} \cap(\operatorname{Span} \pi)^{\perp}
$$

We frequently use related notations like $z+\mathbf{B}_{r}=\left\{z+\xi: \xi \in \mathbf{B}_{r}\right\}$ and $\mathbf{B}_{r}^{\pi}+\mathbf{B}_{r}^{\perp \pi}=$ $\left\{x+y: x \in \mathbf{B}_{r}^{\pi}, y \in \mathbf{B}_{r}^{\perp \pi}\right\}$ (where addition is understood in the sense of the Minkowski sum). In addition, $\mathrm{p}^{\pi}: \mathcal{H} \rightarrow \mathcal{H}$ and $\mathrm{q}^{\pi}: \mathcal{H} \rightarrow \mathcal{H}$ denote the orthogonal projections onto $\operatorname{Span} \pi$ and $(\operatorname{Span} \pi)^{\perp}$, respectively, and the cylinder $\mathbf{C}_{r}^{\pi}$ over $\mathbf{B}_{r}^{\pi}$ is given by

$$
\mathbf{C}_{r}^{\pi}:=\left\{z \in \mathcal{H}: \mathrm{p}^{\pi}(z) \in \mathbf{B}_{r}^{\pi}\right\} .
$$

Finally, we define the $n$-dimensional spherical densities of a Borel measure $\mu$ on $\mathcal{H}$ at a point $z \in \mathcal{H}$ by

$$
\Theta^{* n}(\mu, z):=\limsup _{\varrho \searrow 0} \frac{\mu\left(z+\mathbf{B}_{\varrho}\right)}{\omega_{n} \varrho^{n}}, \quad \Theta_{*}^{n}(\mu, z):=\liminf _{\varrho \searrow 0} \frac{\mu\left(z+\mathbf{B}_{\varrho}\right)}{\omega_{n} \varrho^{n}}
$$

where $\omega_{n}$ stands for the volume of the unit ball in $\mathbb{R}^{n}$. Whenever we have the equality $\Theta^{* n}(\mu, z)=\Theta_{*}^{n}(\mu, z)$, the common value will be denoted by $\Theta^{n}(\mu, z)$.

2.2. Differentiable functions, graphs, area formula, and Jacobian. For a function $g: z+\mathbf{B}_{r}^{\pi} \rightarrow(\operatorname{Span} \pi)^{\perp}$ we write $G: z+\mathbf{B}_{r}^{\pi} \rightarrow z+\mathbf{C}_{r}^{\pi}$ for its graph mapping given by $G(x):=x+g(x)$ (generally the graph mapping is denoted by the corresponding uppercase letter), and we set Graph $g:=\left\{G(x): x \in z+\mathbf{B}_{r}^{\pi}\right\}$. In the following the notations $\mathrm{D} g(x): \operatorname{Span} \pi \rightarrow(\operatorname{Span} \pi)^{\perp}$ and $\mathrm{D} G(x): \operatorname{Span} \pi \rightarrow \mathcal{H}$ will refer to the Fréchet differentials of the maps $g$ and $G$ at $x \in z+\mathbf{B}_{r}^{\pi}$ (notice in particular $\mathrm{D} g(x) \in \mathrm{L}^{\pi}$ ), and we say that $g$ is $\mathrm{C}^{1}$ or $\mathrm{C}^{1, \alpha}$ if the derivative $\mathrm{D} g$ exists everywhere as a continuous or $\alpha$-Hölder-continuous ( $\mathrm{L}^{\pi}$-valued) mapping. We have the orthogonal decomposition $\mathrm{D} G(x) \xi=\xi+\mathrm{D} g(x) \xi$, and the image of $\mathrm{D} G(x)$ is the tangent space to Graph $g$ at $G(x)$ which, compatibly with the notation introduced below for integer rectifiable currents, will be denoted by $\operatorname{Tan}^{(n)}$ (Graph $\left.g, G(x)\right)$.

If $g$ is locally Lipschitz continuous, then the derivatives $\mathrm{D} g(x)$ and $\mathrm{D} G(x)$ exist for $\mathscr{L}^{n}$-a. e. $x \in z+\mathbf{B}_{r}^{\pi}$ by the generalized Rademacher theorem for Lipschitz maps between Hilbert (or even Banach) spaces, see [9, Theorem 5.11.1]. Furthermore, as a particular case of the area formula [28, Corollary 8], [6, Theorem 5.1] we then have

$$
\int_{\operatorname{Graph} g} \varphi \mathrm{d} \mathscr{H}^{n}=\int_{z+\mathbf{B}_{r}^{\pi}} \varphi(G(x)) \mathrm{J}_{n}(\mathrm{D} G(x)) \mathrm{d} \mathscr{L}^{n}(x)
$$

for every bounded Borel function $\varphi$ : Graph $g \rightarrow \mathbb{R}$. Here, the Jacobian $\mathrm{J}_{n}(L)$ of a linear map $L: \operatorname{Span} \pi \rightarrow \mathcal{H}$ can be computed as

$$
\mathrm{J}_{n}(L)=\sqrt{\operatorname{det}\left(\left\langle L \pi_{i}, L \pi_{j}\right\rangle\right)_{i, j=1,2, \ldots, n}}=\left|\bigwedge_{i=1}^{n} L \pi_{i}\right|,
$$

whenever $\pi_{1}, \pi_{2}, \ldots, \pi_{n}$ is an orthonormal basis of Span $\pi$. Choosing the $\pi_{i}$ as eigenvectors of $L^{*} \circ L$, the elementary inequality between the arithmetic and geometric 
means leads to the (optimal) estimate $\mathrm{J}_{n}(L) \leq n^{-\frac{n}{2}}|L|^{n}$. We also record the following useful expansion, whose proof resembles the computations of Section 2.5:

$$
\left|\mathrm{J}_{n}(\mathrm{Id}+\varepsilon L)^{2}-1-\varepsilon \operatorname{trace}_{\pi}(L)\right| \leq C \varepsilon^{2}\left(1+|L|^{2 n}\right) \quad \forall \varepsilon \in(0,1] .
$$

Here, Id: $\operatorname{Span} \pi \rightarrow \mathcal{H}$ is the embedding, the constant $C$ depends only on $n$, and $\operatorname{trace}_{\pi}(L)$ is defined by

$$
\operatorname{trace}_{\pi}(L):=\sum_{i=1}^{n}\left\langle L \pi_{i}, \pi_{i}\right\rangle .
$$

2.3. General issues about currents. In the sequel we follow widely [5]. We recall that an $n$-dimensional current $T$ in $\mathcal{H}$ is defined as an $(1+n)$-linear functional

$$
\operatorname{Lip}_{\mathrm{b}}(\mathcal{H}) \times \operatorname{Lip}(\mathcal{H})^{n} \ni\left(\varphi, \psi_{1}, \ldots, \psi_{n}\right) \mapsto T\left(\varphi, \psi_{1}, \ldots, \psi_{n}\right) \in \mathbb{R}
$$

satisfying suitable continuity and locality axioms, where $\operatorname{Lip}_{\mathrm{b}}$ denotes the class of bounded Lipschitz functions. Currents with finite mass are characterized by the existence of a positive, finite Borel measure $\mu$ satisfying

$$
\left|T\left(\varphi, \psi_{1}, \ldots, \psi_{n}\right)\right| \leq \prod_{i=1}^{n} \operatorname{Lip}\left(\psi_{i}\right) \int_{\mathcal{H}}|\varphi| \mathrm{d} \mu \quad \forall \varphi \in \operatorname{Lip}_{\mathrm{b}}(\mathcal{H}), \psi_{1}, \ldots, \psi_{n} \in \operatorname{Lip}(\mathcal{H})
$$

The smallest measure $\mu$ with this property is denoted by $\|T\|$, the mass is $\mathbf{M}(T):=$ $\|T\|(\mathcal{H})$, and the class of currents with finite mass is called $\mathbf{M}_{n}(\mathcal{H})$.

The class $\mathbf{N}_{n}(\mathcal{H})$ of normal currents is, as in the classical Federer-Fleming theory,

$$
\mathbf{N}_{n}(\mathcal{H}):=\left\{T \in \mathbf{M}_{n}(\mathcal{H}): \partial T \in \mathbf{M}_{n}(\mathcal{H})\right\},
$$

where, at the level of general $n$-dimensional currents $T$, the boundary operator is defined by

$$
\partial T\left(\varphi, \psi_{1}, \ldots, \psi_{n-1}\right):=T\left(1, \varphi, \psi_{1}, \ldots, \psi_{n-1}\right) .
$$

We recall that when $\mathcal{H} \cong \mathbb{R}^{N}$ is finite-dimensional, the class $\mathbf{N}_{n}(\mathcal{H})$ and the class $\mathscr{R}_{n}(\mathcal{H})$ of rectifiable currents defined below are fully consistent with those of the Federer-Fleming theory. As a matter of fact, in the rest of the paper the maps $\psi_{i}$ will often be linear maps.

For currents $T$ with finite mass (the only ones we shall consider) the action can be canonically extended to bounded Borel functions $\varphi$. Furthermore, we adopt the conventional notation

$$
T\left(\varphi \bigwedge_{i=1}^{n} \mathrm{~d} \psi_{i}\right):=T\left(\varphi, \psi_{1}, \ldots, \psi_{n}\right),
$$

justified by the fact that the axioms imply the chain rule

$$
T\left(\varphi \bigwedge_{i=1}^{n} \mathrm{~d}\left(\chi_{i} \circ \psi\right)\right)=T\left(\varphi(\operatorname{det} \nabla \chi) \circ \psi \bigwedge_{i=1}^{n} \mathrm{~d} \psi_{i}\right) \quad \text { for all } \chi \in C^{1}\left(\mathbb{R}^{n} ; \mathbb{R}^{n}\right) \text { Lipschitz }
$$

(see [5, Theorem 3.5]) and, in particular, the alternating property. 
We also record the following statement, which follows from the codimension 1 case of the slicing theorem [5, Theorem 5.6]: Given $T \in \mathbf{N}_{n}(\mathcal{H}), \psi \in \operatorname{Lip}(\mathcal{H})$, and $-\infty \leq s_{1} \leq s_{2} \leq \infty$, the function $t \mapsto \mathbf{M}\left(\partial(T\llcorner\{\psi>t\}))\right.$ is $\mathscr{L}^{1}$-measurable and $\mathscr{L}^{1}$-a. e. finite, and we have

$$
\begin{gathered}
\| T\left\llcorner\mathrm{~d} \psi \|\left(\left\{s_{1}<\psi<s_{2}\right\}\right)=\int_{s_{1}}^{s_{2}} \mathbf{M}\left(\partial(T\llcorner\{\psi>t\})) \mathrm{d} \mathscr{L}^{1}(t)\right.\right. \\
\text { provided that }(\partial T)\left\llcorner\left\{\psi>s_{1}\right\} \equiv 0 .\right.
\end{gathered}
$$

2.4. Rectifiable and integral currents in $\mathcal{H}$. Following [5] again, we call $T \in$ $\mathbf{M}_{n}(\mathcal{H})$ rectifiable if $\|T\|$ is absolutely continuous with respect to $\mathscr{H}^{n}$ and concentrated on a countably $\mathscr{H}^{n}$-rectifiable set. The class of rectifiable currents will be denoted by $\mathscr{R}_{n}(\mathcal{H})$. Inside this class we can also single out the class $\mathscr{I}_{n}(\mathcal{H})$ of integerrectifiable currents, defined by the property

$$
\Theta^{n}(\|T\|, z) \in \mathbb{N} \quad \text { for }\|T\| \text {-a.e. } z \in \mathcal{H} .
$$

Finally, the class $\mathbf{I}_{n}(\mathcal{H})$ of integral currents, which will be our main object of investigation, is simply $\mathscr{I}_{n}(\mathcal{H}) \cap \mathbf{N}_{n}(\mathcal{H})$.

Next we recall some results of [5, Section 9] about rectifiable currents, specialized to the present Hilbertian case. By [5, Theorem 9.1], for every $T \in \mathscr{R}_{n}(\mathcal{H})$ there exist a countably $\mathscr{H}^{n}$-rectifiable subset $\mathrm{S}_{T}$ of $\mathcal{H}$, a Borel function $\theta_{T}: \mathrm{S}_{T} \rightarrow(0, \infty)$ with $\int_{\mathrm{S}_{T}} \theta_{T} \mathrm{~d} \mathscr{H}^{n}<\infty$, and an orientation $\vec{T}$ of $\mathrm{S}_{T}$ such that we have

$$
T\left(\varphi \bigwedge_{i=1}^{n} \mathrm{~d} \psi_{i}\right)=\int_{\mathrm{S}_{T}} \varphi\left\langle\bigwedge_{i=1}^{n} \mathrm{D}^{\mathrm{S}_{T}} \psi_{i}, \vec{T}\right\rangle \theta_{T} \mathrm{~d} \mathscr{H}^{n}
$$

for all $(\varphi, \psi) \in \operatorname{Lip}_{\mathrm{b}}(\mathcal{H}) \times \operatorname{Lip}(\mathcal{H})^{n}$. Here, the orientation $\vec{T}: \mathrm{S}_{T} \rightarrow \Lambda_{n}(\mathcal{H})$ is a Borel function such that $\vec{T}(z)$ is a unit simple $n$-vector over the approximative tangent space $\operatorname{Tan}^{(n)}\left(\mathrm{S}_{T}, z\right)$, in other words $\vec{T}(z)$ is an $n$-plane with $\operatorname{Span} \vec{T}(z)=\operatorname{Tan}^{(n)}\left(\mathrm{S}_{T}, z\right)$, for $\mathscr{H}^{n}$-a. e. $z \in \mathrm{S}_{T}$. Moreover, $\mathrm{D}^{\mathrm{S}_{T}} \psi_{i}$ is the tangential differential of $\psi_{i}$ along $\mathrm{S}_{T}$. In [5, Section 9], the differential $\mathrm{D}_{T}^{\mathrm{S}} \psi_{i}(z): \operatorname{Tan}^{(n)}\left(\mathrm{S}_{T}, z\right) \rightarrow \mathbb{R}$ is understood as a 1-covector over $\operatorname{Tan}^{(n)}\left(\mathrm{S}_{T}, z\right)$, and correspondingly the angle brackets in (2.10) denote the usual evaluation of an $n$-covector on an $n$-vector. In our situation, taking into account the Hilbertian structure of $\mathcal{H}$, we identify $\mathrm{D}^{\mathrm{S}_{T}} \psi_{i}(z)$ with a 1 -vector over $\operatorname{Tan}^{(n)}\left(\mathrm{S}_{T}, z\right)$ via the Riesz isomorphism, and correspondingly we reinterpret the angle brackets as the inner product of (2.1).

A triplet $\left(\mathrm{S}_{T}, \theta_{T}, \vec{T}\right)$, for which (2.10) holds, is - up to sets $\mathscr{H}^{n}$-measure zero - uniquely determined by $T$, and for the following we can indeed fix the canonical choices

$$
\mathrm{S}_{T}:=\left\{z \in \mathcal{H}: \Theta^{* n}(\|T\|, z)>0\right\}, \quad \theta_{T}(z):=\Theta^{* n}(\|T\|, z) .
$$

Moreover, by [5, Theorem 9.1] we know that every suitable triplet $(S, \vartheta, \tau)$ originates from a current in $T \in \mathscr{R}_{n}(\mathcal{H})$ (in the sense that $\left(\mathrm{S}_{T}, \theta_{T}, \vec{T}\right)$ equals $(S, \vartheta, \tau)$ up to 
$\mathscr{H}^{n}$-negligible sets), and we will denote this current by $\vartheta \llbracket S \rrbracket^{\tau}$. Here, the superscript $\tau$ is often omitted when there is a canonical choice of the orientation. In particular, for balls $z+\mathbf{B}_{r}^{\pi}$ in an $n$-plane $\pi$ we always understand $\llbracket z+\mathbf{B}_{r}^{\pi} \rrbracket:=\llbracket z+\mathbf{B}_{r}^{\pi} \rrbracket^{\pi} \in \mathbf{I}_{n}(\mathcal{H})$. Moreover, when we write $\pi=\bigwedge_{i=1}^{n} \pi_{i}$ with orthonormal $\pi_{1}, \pi_{2}, \ldots, \pi_{n} \in \operatorname{Span} \pi$ and $g: z+\mathbf{B}_{r}^{\pi} \rightarrow(\operatorname{Span} \pi)^{\perp}$ is a locally Lipschitz continuous function, $\bigwedge_{i=1}^{n} \mathrm{D} G(x) \pi_{i}$ is an $n$-vector over $\operatorname{Tan}^{(n)}(\operatorname{Graph} g, G(x))$ for $\mathscr{L}^{n}$-a. e. $x \in z+\mathbf{B}_{r}^{\pi}$. This $n$-vector depends only on $\pi$, but not on the choice of the $\pi_{i}$, its modulus is $\mathrm{J}_{n}(\mathrm{D} G(x)$ ) (compare with (2.5)), and when $\mathscr{H}^{n}($ Graph $g)<\infty$ we can use it to endow Graph $g$ with a canonical orientation: when dealing with the integer-rectifiable current «Graph $g \rrbracket$ we always understand

$$
\overrightarrow{\llbracket \operatorname{Graph} g \rrbracket}(G(x))=\frac{\bigwedge_{i=1}^{n} \mathrm{D} G(x) \pi_{i}}{\mathrm{~J}_{n}(\mathrm{D} G(x))} \quad \text { for } \mathscr{H}^{n} \text {-a. e. point } G(x) \text {. }
$$

In case of a globally Lipschitz continuous $g$ we finally infer via the area formula that $\llbracket$ Graph $g \rrbracket=G_{\sharp} \llbracket z+\mathbf{B}_{r}^{\pi} \rrbracket$.

With the previous notation the characterization of mass [5, Theorem 9.5] yields

$$
\|T\|=\theta_{T} \mathscr{H}^{n}\left\llcorner\mathrm{~S}_{T}\right.
$$

for every $T \in \mathscr{R}_{n}(\mathcal{H})$, and in combination with (2.10) we get

$$
T\left(\varphi \bigwedge_{i=1}^{n} \mathrm{~d} \psi_{i}\right)=\int_{\mathcal{H}} \varphi\left\langle\bigwedge_{i=1}^{n} \mathrm{D}^{\mathrm{S}_{T}} \psi_{i}, \vec{T}\right\rangle \mathrm{d}\|T\|
$$

for all $(\varphi, \psi) \in \operatorname{Lip}_{\mathrm{b}}(\mathcal{H}) \times \operatorname{Lip}(\mathcal{H})^{n}$. In view of this formula it appears reasonable to extend the action of $T$ to arbitrary bounded Borel functions $\chi: \mathcal{H} \rightarrow \Lambda_{n}(\mathcal{H})$ by

$$
T(\chi):=\int_{\mathcal{H}}\langle\chi, \vec{T}\rangle \mathrm{d}\|T\|
$$

We record in particular that with this convention we have

$$
T(\chi) \equiv 0 \quad \text { whenever } T \text { has zero boundary and } \chi \text { is a constant } n \text {-vector. }
$$

We provide a simple proof of (2.16) for the reader's convenience: We first observe that it suffices to show (2.16) for simple $n$-vectors $\chi=\bigwedge_{i=1}^{n} \chi_{i}$. Setting $\mathrm{p}_{i}^{\chi}(z):=\left\langle\chi_{i}, z\right\rangle$ the tangential derivative $\mathrm{D}^{\mathrm{S}_{T}} \mathrm{p}_{i}^{\chi}(z)$ is the projection of $\chi_{i}$ onto $\operatorname{Tan}^{(n)}\left(\mathrm{S}_{T}, z\right)$, and as a consequence we have $\langle\chi, \vec{T}\rangle=\left\langle\bigwedge_{i=1}^{n} \mathrm{D}^{\mathrm{S}_{T}} \mathrm{p}_{i}^{\chi}, \vec{T}\right\rangle$. With (2.14) and (2.15) we can thus conclude $T(\chi)=T\left(1 \bigwedge_{i=1}^{n} \mathrm{dp}_{i}^{\chi}\right)=0$, where the last equality follows from $\partial T=0$.

Finally, consider a current $T \in \mathscr{R}_{n}(\mathcal{H})$ and a cylinder $z+\mathbf{C}_{r}^{\pi}$, where $\pi$ is an $n$-plane (i. e. an oriented $n$-dimensional subspace) in $\mathcal{H}, z$ is a point in $\mathcal{H}$, and $r>0$ is a positive radius. The cylindrical excess of $T$ in the cylinder $z+\mathbf{C}_{r}^{\pi}$ is then the quantity

$$
\mathbf{E}(T, z, r, \pi):=r^{-n} \int_{z+\mathbf{C}_{r}^{\pi}}|\vec{T}-\pi|^{2} \mathrm{~d}\|T\|,
$$

which measures the deviation of $T$ from $\pi$ and which will play a major role in this article. Abbreviating $T_{z, r}^{\pi}=T\left\llcorner\left(z+\mathbf{C}_{r}^{\pi}\right)\right.$ and using the same arguments as for (2.16), 
we obtain

$$
\int_{z+\mathbf{C}_{r}^{\pi}}\langle\pi, \vec{T}\rangle \mathrm{d}\|T\|=T_{z, r}^{\pi}\left(1 \bigwedge_{i=1}^{n} \mathrm{~d} \mathrm{p}_{i}^{\pi}\right)=\left(\mathrm{p}^{\pi}\right)_{\sharp} T_{z, r}^{\pi}\left(1 \bigwedge_{i=1}^{n} \mathrm{dp}_{i}^{\pi}\right)=\left(\mathrm{p}^{\pi}\right)_{\sharp} T_{z, r}^{\pi}(\pi) .
$$

In particular, since $|\vec{T}|=|\pi|=1$, we can write $\mathbf{E}$ in the alternative form

$$
\mathbf{E}(T, z, r, \pi)=\frac{2}{r^{n}}\left[\|T\|\left(z+\mathbf{C}_{r}^{\pi}\right)-\left(\mathrm{p}^{\pi}\right)_{\sharp} T_{z, r}^{\pi}(\pi)\right] .
$$

Finally, if $T$ is locally mass-minimizing in $\Omega$ we have the monotonicity property (see Appendix B for an outline of the relevant arguments)

$$
r \mapsto \frac{\|T\|\left(z+\mathbf{B}_{r}\right)}{\omega_{n} r^{n}} \text { is non-decreasing in }(0, \operatorname{dist}(z, \partial \Omega))
$$

for all $z \in \Omega$. As a standard consequence, we obtain the lower mass estimate

$$
\|T\|\left(z+\mathbf{B}_{r}\right) \geq \Theta^{* n}(\|T\|, z) \omega_{n} r^{n} \quad \text { whenever } z \in \Omega \text { and } r \in(0, \operatorname{dist}(z, \partial \Omega)),
$$

and the existence and upper semicontinuity of $\Theta^{n}(\|T\|, \cdot)$ in all of $\Omega$. In turn, these properties of $\Theta^{n}(\|T\|, \cdot)$ and (2.9) imply that the set $\left\{z \in \Omega: \Theta^{n}(\|T\|, z) \geq 1\right\}$ is closed in $\Omega$ and coincides with $\Omega \cap$ spt $T$. Therefore, in the applications of (2.20) with $z \in \Omega \cap \operatorname{spt} T$, the density on the right hand side is controlled from below by 1 .

Finally, if $\Gamma$ is a subset of $\Omega \cap$ spt $T$ with positive distance from $\partial \Omega$, then we claim that $\Gamma$ can be covered, for every given $\varepsilon \in(0,2 \operatorname{dist}(\Gamma, \partial \Omega))$, by finitely many open balls with centers in $\Gamma$ and with radius $\varepsilon$. Indeed, by the Hausdorff maximal principle there exists a maximal set $\mathcal{C} \subset \Gamma$ of centers such that $|\widetilde{z}-z| \geq \varepsilon$ holds whenever $z \neq \widetilde{z}$ in $\mathcal{C}$; then the balls $z+\mathbf{B}_{\varepsilon / 2}$ with $z \in \mathcal{C}$ are disjoint and (2.20) gives $\|T\|\left(z+\mathbf{B}_{\varepsilon / 2}\right) \geq \omega_{n}(\varepsilon / 2)^{n}$, so that $\mathcal{C}$ need be finite, and thus the balls $z+\mathbf{B}_{\varepsilon}$ with $z \in \mathcal{C}$ form the claimed cover. This argument shows that $\Gamma$ is totally bounded, and thus every closed subset of $\Omega \cap \operatorname{spt} T$ with positive distance from $\partial \Omega$ is necessarily compact.

2.5. Some multilinear and Grassmannian algebra. In what follows we assume again that $\pi$ is an $n$-plane in $\mathcal{H}$, and we represent $\pi$ as $\bigwedge_{i=1}^{n} \pi_{i}$ with a fixed orthonormal basis $\pi_{1}, \pi_{2}, \ldots, \pi_{n}$ of Span $\pi$. Moreover, $s$ always stands for a real parameter, and $\ell, \ell_{0}: \operatorname{Span} \pi \rightarrow(\operatorname{Span} \pi)^{\perp}$ are linear maps with graph mappings $L$ and $L_{0}$, respectively. With these notations we will now collect some (in)equalities for the $n$-vector $\bigwedge_{i=1}^{n} L \pi_{i}$, where all constants, here generically denoted by $C$, are understood to depend only on $n$.

Recalling that $L \pi_{i}=\pi_{i}+\ell \pi_{i}$, we expand $\bigwedge_{i=1}^{n} L \pi_{i}$ correspondingly and, for $k \in$ $\{1,2, \ldots, n\}$, we write $[\ell]_{k}^{\pi}$ for the sum of those terms in the expansion which are $k$-linear in $\ell$. In particular, the constant term is $[\ell]_{0}^{\pi}=\bigwedge_{i=1}^{n} \pi_{i}=\pi$, the linear one is $[\ell]_{1}^{\pi}=\sum_{i=1}^{n}\left[\pi_{1} \wedge \ldots \wedge \pi_{i-1} \wedge \ell \pi_{i} \wedge \pi_{i+1} \wedge \ldots \wedge \pi_{n}\right]$, and for the higher-order terms we just record

$$
\left|[\ell]_{k}^{\pi}\right| \leq C|\ell|^{k}
$$


Evidently we then have the following basic error estimates:

$$
\begin{gathered}
\left|\bigwedge_{i=1}^{n} L \pi_{i}-\pi\right| \leq C\left[|\ell|+|\ell|^{n}\right], \\
\left|\bigwedge_{i=1}^{n} L \pi_{i}-\pi-[\ell]_{1}^{\pi}\right| \leq C\left[|\ell|^{2}+|\ell|^{n}\right] .
\end{gathered}
$$

Now we observe that the $\pi_{i}$ are mutually orthonormal and orthogonal to each $\ell \pi_{i}$ and $\ell_{0} \pi_{i}$ (remember that $\ell$ and $\ell_{0}$ map $\operatorname{Span} \pi$ into $\left.(\operatorname{Span} \pi)^{\perp}\right)$. Using these orthogonality relations in $(2.1)$ one finds ${ }^{3}\left\langle[\ell]_{1}^{\pi},\left[\ell_{0}\right]_{1}^{\pi}\right\rangle=\left\langle\ell, \ell_{0}\right\rangle$ (in particular $\left|[\ell]_{1}^{\pi}\right|=|\ell|$ ) and $\left\langle[\ell]_{k}^{\pi},\left[\ell_{0}\right]_{l}^{\pi}\right\rangle=0$ for $k \neq l$, and all in all we get

$$
\begin{gathered}
\left\langle\bigwedge_{i=1}^{n} L \pi_{i}, \bigwedge_{j=1}^{n} L_{0} \pi_{j}\right\rangle=1+\left\langle\ell, \ell_{0}\right\rangle+\sum_{k=2}^{n}\left\langle[\ell]_{k}^{\pi},\left[\ell_{0}\right]_{k}^{\pi}\right\rangle, \\
\left|s \bigwedge_{i=1}^{n} L \pi_{i}-\pi\right|^{2}=(s-1)^{2}+s^{2}|\ell|^{2}+s^{2} \sum_{k=2}^{n}\left|[\ell]_{k}^{\pi}\right|^{2} .
\end{gathered}
$$

Recalling (2.5) we also infer

$$
\mathrm{J}_{n}(L)^{2}=1+|\ell|^{2}+\sum_{k=2}^{n}\left|[\ell]_{k}^{\pi}\right|^{2}
$$

from which we conclude $\mathrm{J}_{n}(L) \geq 1$ and (taking also (2.21) into account)

$$
\left|1-\frac{1}{\mathrm{~J}_{n}(L)}\right|=\frac{\mathrm{J}_{n}(L)^{2}-1}{\mathrm{~J}_{n}(L)\left(\mathrm{J}_{n}(L)+1\right)} \leq C \min \left\{\sum_{k=1}^{n}|\ell|^{2 k}, 1\right\} \leq C \min \left\{|\ell|^{2}, 1\right\} .
$$

Next we record two simple algebraic lemmas which relate different ways of measuring the distance between $n$-planes.

Lemma 2.1. Given two n-planes $\pi$ and $\varpi$ in $\mathcal{H}$ with $|\varpi-\pi| \leq \frac{1}{2}$ there exists a linear $\operatorname{map} \ell: \operatorname{Span} \pi \rightarrow(\operatorname{Span} \pi)^{\perp}$ with

$$
\text { Graph } \ell=\operatorname{Span} \varpi \quad \text { and } \quad|\ell| \leq 2|\varpi-\pi| .
$$

Proof. We first observe Span $\varpi \cap(\operatorname{Span} \pi)^{\perp}=\{0\}$ (for otherwise we would have $\langle\varpi, \pi\rangle=0$ and $\left.|\varpi-\pi|^{2}=2\right)$. This observation implies that the restriction of $\mathrm{p}^{\pi}$ to Span $\varpi$ maps Span $\varpi$ one-to-one onto $\operatorname{Span} \pi$, and the inverse of this mapping minus the identity gives a linear $\ell: \operatorname{Span} \pi \rightarrow(\operatorname{Span} \pi)^{\perp}$ with $\operatorname{Graph} \ell=\operatorname{Span} \varpi$.

\footnotetext{
${ }^{3}$ Indeed, the formula for $\left\langle[\ell]_{1}^{\pi},\left[\ell_{0}\right]_{1}^{\pi}\right\rangle$ is quite easy to check, while the general orthogonality relation between $[\ell]_{k}^{\pi}$ and $\left[\ell_{0}\right]_{l}^{\pi}$ can be verified as follows: when $\Sigma_{k}$ and $\Sigma_{l}$, respectively, are simple summands of $[\ell]_{k}^{\pi}$ and $\left[\ell_{0}\right]_{l}^{\pi}$ with $k<l \leq n$, then there is an index $i$ such that $\Sigma_{k}$ contains a factor $\pi_{i}$, while $\Sigma_{l}$ contains $\ell_{0} \pi_{i}$ instead. Thus, $\pi_{i}$ is actually orthogonal to all the factors in $\Sigma_{l}$, and recalling the determinant structure (2.1) this suffices to conclude $\left\langle\Sigma_{k}, \Sigma_{l}\right\rangle=0$.
} 
Now we fix again an orthonormal basis $\pi_{1}, \pi_{2}, \ldots, \pi_{n}$ of $\operatorname{Span} \pi$. As there are only two constant orientations of Span $\varpi$, we have

$$
\varpi=\frac{\bigwedge_{i=1}^{n} L \pi_{i}}{\mathrm{~J}_{n}(L)} \quad \text { or } \quad \varpi=-\frac{\bigwedge_{i=1}^{n} L \pi_{i}}{\mathrm{~J}_{n}(L)} .
$$

Here, the second case however cannot occur, since (2.25) with $s=-1 / \mathrm{J}_{n}(L)$ would then give the contradiction $\frac{1}{4} \geq|\varpi-\pi|^{2} \geq\left(\frac{1}{\mathrm{~J}_{n}(L)}+1\right)^{2}$. In the first case (2.25) yields the estimate

$$
\frac{1}{4} \geq|\varpi-\pi|^{2} \geq\left(\frac{1}{\mathrm{~J}_{n}(L)}-1\right)^{2}+\frac{|\ell|^{2}}{\mathrm{~J}_{n}(L)^{2}}
$$

from which we subsequently deduce, $1-\frac{1}{\mathrm{~J}_{n}(L)} \leq \frac{1}{2}$, hence $\mathrm{J}_{n}(L) \leq 2$ and finally $|\ell| \leq 2|\varpi-\pi|$.

Lemma 2.2. Given two n-planes $\pi$ and $\varpi$ in $\mathcal{H}$ there holds

$$
\left|\mathrm{p}^{\varpi}(z)-\mathrm{p}^{\pi}(z)\right| \leq 8 n|\varpi-\pi||z| \quad \text { for all } z \in \mathcal{H} .
$$

Proof. We assume $|\varpi-\pi| \leq \frac{1}{2}$ (otherwise the claim is trivially true), and we use the $\ell$ of Lemma 2.1 and an orthonormal basis $\pi_{1}, \pi_{2}, \ldots, \pi_{n}$ of $\operatorname{Span} \pi$ which consists of eigenvectors of $L^{*} \circ L$. Setting $\varpi_{i}:=\frac{L \pi_{i}}{\left|L \pi_{i}\right|}$ we then find an orthonormal basis $\varpi_{1}, \varpi_{2}, \ldots, \varpi_{n}$ of Span $\varpi$. We have

$$
\left|\varpi_{i}-\pi_{i}\right|=\left|\frac{\ell \pi_{i}+\pi_{i}\left(1-\left|\pi_{i}+\ell \pi_{i}\right|\right)}{\left|\pi_{i}+\ell \pi_{i}\right|}\right| \leq 2\left|\ell \pi_{i}\right| \stackrel{(2.28)}{\leq} 4|\varpi-\pi| .
$$

Now we can compute

$$
\left|\mathrm{p}^{\varpi}(z)-\mathrm{p}^{\pi}(z)\right|=\left|\sum_{i=1}^{n}\left[\left\langle z, \varpi_{i}\right\rangle \varpi_{i}-\left\langle z, \pi_{i}\right\rangle \pi_{i}\right]\right| \leq 2 \sum_{i=1}^{n}\left|\varpi_{i}-\pi_{i}\right||z| \leq 8 n|\varpi-\pi||z| .
$$

We will mostly apply the previous considerations to the differential of locally Lipschitz functions $g: \mathbf{B}_{\sigma}^{\pi} \rightarrow(\operatorname{Span} \pi)^{\perp}$ with $\mathscr{H}^{n}($ Graph $g)<\infty$. In this case, at any point $x$ of differentiability of $g$, we set $\ell=\mathrm{D} g(x)$ for $x \in \mathbf{B}_{\sigma}^{\pi}$ and we observe that $L=\mathrm{D} G(x)$ (remember that $G$ and $L$ denote the graph mappings of $g$ and $\ell$, respectively). Recalling (2.12), we combine (2.22), (2.23), and (2.27) to achieve the following estimates (which are all understood to hold $\mathscr{L}^{n}$-a. e. on $\mathbf{B}_{\sigma}^{\pi}$ )

$$
\begin{aligned}
& |\overrightarrow{\llbracket \operatorname{Graph} g \rrbracket \circ G}-\pi| \leq C \min \{|\mathrm{D} g|, 1\}, \\
& \left|\overrightarrow{\llbracket \text { Graph } g \rrbracket} \circ G-\pi-[\mathrm{D} g]_{1}^{\pi}\right| \leq C|\mathrm{D} g| \min \{|\mathrm{D} g|, 1\} \text {. }
\end{aligned}
$$

Moreover, (2.12) and (2.25) with $s=1 / \mathrm{J}_{n}(\mathrm{D} G)$ tell us

$$
|\overrightarrow{\llbracket \text { Graph } g \rrbracket} \circ G-\pi|^{2} \mathrm{~J}_{n}(\mathrm{D} G)^{2}=\left(1-\mathrm{J}_{n}(\mathrm{D} G)\right)^{2}+|\mathrm{D} g|^{2}+\sum_{k=2}^{n}\left|[\mathrm{D} g]_{k}^{\pi}\right|^{2} \geq|\mathrm{D} g|^{2}
$$




\section{Codimension-Free elliptic estimates}

We now write $\mathbf{B}_{\sigma}^{n}$ for the open ball with center 0 and radius $\sigma$ in $\mathbb{R}^{n}$, and we continue to use $|\cdot|$ for Hilbertian norms, here specifically for the Hilbert-Schmidt norm of the matrix-valued derivatives. With this notation we state an interior bound, an interior Lipschitz estimate, and a global $\mathrm{L}^{p}$-estimate for the gradients of harmonic functions.

Lemma 3.1. For every harmonic function $h: \mathbf{B}_{\sigma}^{n} \rightarrow \mathbb{R}^{N}$ and $0<\eta<\sigma$ one has

$$
\begin{gathered}
\sup _{\mathbf{B}_{\eta}^{n}}|\mathrm{D} h|^{2} \leq \frac{C}{(\sigma-\eta)^{n}} \int_{\mathbf{B}_{\sigma}^{n}}|\mathrm{D} h|^{2} \mathrm{~d} \mathscr{L}^{n}, \\
|\mathrm{D} h(y)-\mathrm{D} h(x)|^{2} \leq C \frac{|y-x|^{2}}{(\sigma-\eta)^{n+2}} \int_{\mathbf{B}_{\sigma}^{n}}|\mathrm{D} h|^{2} \mathrm{~d} \mathscr{L}^{n} \quad \text { for } x, y \in \mathbf{B}_{\eta}^{n},
\end{gathered}
$$

where $C$ depends only on $n$ and is in particular independent of $N$. Moreover, if $h$ is harmonic and contained in a Dirichlet class $f+\mathrm{W}_{0}^{1,2}\left(\mathbf{B}_{\sigma}^{n}, \mathbb{R}^{N}\right)$ with $f \in \mathrm{W}^{1, \infty}\left(\mathbf{B}_{\sigma}^{n}, \mathbb{R}^{N}\right)$, then for all $1<p<\infty$ one has

$$
\int_{\mathbf{B}_{\sigma}^{n}}|\mathrm{D} h|^{p} \mathrm{~d} \mathscr{L}^{n} \leq C \sigma^{n} \underset{\mathbf{B}_{\sigma}^{n}}{\operatorname{essip}}|\mathrm{D} f|^{p},
$$

where $C$ depends only on $n$ and $p$ and is again independent of $N$.

All three estimates are classical, except for the claim that the constants do not depend on $N$. In case of (3.1) and (3.2) the latter claim can however be checked very easily: one may either revisit a classical proof ${ }^{4}$ of these estimates or add up the respective estimates for the $N$ single component functions of $h$. In contrast, for (3.3) (in the non-trivial case $p>2$ ) the analogous summation over the component functions does not give an $N$-independent constant (the basic obstruction is that the $\mathrm{L}^{p}$-structure is not compatible with the inner-product norm $|\cdot|$ ), but revisiting the classical proofs it is still possible to justify our claim. In the following we will suggest a slightly modified approach to (3.3), which is specific for the Dirichlet problem on balls and has the advantage of reducing the portion of the classical argument which needs to be revisited.

Proof of (3.3) with $N$-independent constant $C$. We assume $n \geq 3$, as the case $n=1$ is trivial and the case $n=2$ is covered by a minor adaption (with modified functions $\Gamma$ and $G$ ) of the following arguments. Moreover we will assume that $\sigma$ equals 1 and that $f$ vanishes on $\mathbf{B}_{1 / 2}^{n}$. To justify the last simplifying assumption we argue as follows: reducing first to $f(0)=0$ we replace $f$ with $\varphi f$, where $\varphi$ is a smooth function, which vanishes on $\mathbf{B}_{1 / 2}^{n}$, takes the constant value 1 near the boundary of $\mathbf{B}_{1}^{n}$, and satisfies

\footnotetext{
${ }^{4} \mathrm{~A}$ very short and elementary proof indeed starts from the observation that a mollification of $\mathrm{D} h$ with a smooth and rotationally symmetric kernel, supported in $\mathbf{B}_{\sigma-\eta}^{n}$, coincides with $\mathrm{D} h$ on $\mathbf{B}_{\eta}^{n}$ by the mean value property. From this equality one can easily obtain (3.1). Furthermore, differentiating the equality one finds an estimate for $\sup _{\mathbf{B}_{\eta}^{n}}\left|\mathrm{D}^{2} h\right|^{2}$, which readily implies (3.2).
} 
$\sup _{\mathbf{B}_{1}^{n}}|\varphi|+\sup _{\mathbf{B}_{1}^{n}}|\mathrm{D} \varphi| \leq 4$. We then have ess $\sup _{\mathbf{B}_{1}^{n}}|\mathrm{D}(\varphi f)| \leq 4 \operatorname{ess}_{\sup _{\mathbf{B}_{1}^{n}}}|\mathrm{D} f|$; thus it indeed suffices to prove the claim with $\varphi f$ in place of $f$.

Keeping in mind the preceding reductions we now perform a partial integration in Green's representation formula for solutions of the Poisson equation (see for instance [22, Chapters 2.4,2.5] or [18, Chapter 2.2.4]). The resulting formula remains valid for right hand sides in $W^{-1,2}\left(\mathbf{B}_{1}^{n}, \mathbb{R}^{N}\right.$ ), and when we apply it to $h-f$ (which solves the Laplace equation with zero boundary datum and right hand side $-\Delta f$ ) we achieve

$$
h(x)-f(x)=\int_{\mathbf{B}_{1}^{n}} \mathrm{D} f(y) \mathrm{D}_{y} \mathrm{G}(x, y) \mathrm{d} \mathscr{L}^{n}(y) \quad \text { for } x \in \mathbf{B}_{1}^{n},
$$

where $\mathrm{G}$ is the Green's function of the unit ball $\mathbf{B}_{1}^{n}$ in $\mathbb{R}^{n}$ and $\mathrm{D} f(y) \in \mathbb{R}^{N \times n}$ is multiplied with $\mathrm{D}_{y} \mathrm{G}(x, y) \in \mathbb{R}^{n}$ in the sense of the usual matrix-vector-product. In our simple case $\mathrm{G}$ is in fact given by the explicit formula

$$
\mathrm{G}(x, y)=\Gamma(x-y)-|y|^{2-n} \Gamma\left(x-y^{*}\right) \quad \text { for } x, y \in \mathbf{B}_{1}^{n},
$$

where we used the abbreviations $\Gamma(x):=\frac{-1}{(n-2) n \omega_{n}}|x|^{2-n}$ and $y^{*}=|y|^{-2} y$ for the fundamental solution of Laplace's equation and the reflection at the unit sphere, respectively. Calculating $\mathrm{D}_{y} \mathrm{G}$ we can rewrite (3.4) as

$$
\begin{aligned}
h(x)-f(x)= & -\int_{\mathbf{B}_{1}^{n}} \operatorname{Df}(y) \mathrm{D} \Gamma(x-y) \mathrm{d} \mathscr{L}^{n}(y) \\
& +\int_{\mathbf{B}_{1}^{n}} \operatorname{Df} f(y) \frac{|y|^{2} \mathrm{Id}_{n \times n}-2 y \otimes y}{|y|^{n+2}} \mathrm{D} \Gamma\left(x-y^{*}\right) \mathrm{d} \mathscr{L}^{n}(y) \\
& +(n-2) \int_{\mathbf{B}_{1}^{n}} \operatorname{D} f(y) \frac{y}{|y|^{n}} \Gamma\left(x-y^{*}\right) \mathrm{d} \mathscr{L}^{n}(y)
\end{aligned}
$$

for $x \in \mathbf{B}_{1}^{n}$. Now we introduce $g: \mathbb{R}^{n} \rightarrow \mathbb{R}^{N \times n}$ and $\widetilde{g}: \mathbb{R}^{n} \rightarrow \mathbb{R}^{N}$ by

$$
g(y):= \begin{cases}-\mathrm{D} f(y) & \text { for }|y|<1 \\ \mathrm{D} f\left(y^{*}\right) \frac{\left|y^{*}\right|^{2} \operatorname{Id}_{n \times n}-2 y^{*} \otimes y^{*}}{\left|y^{*}\right|^{n+2}|y|^{2 n}} & \text { for }\left|y^{*}\right|<1\end{cases}
$$

and

$$
\widetilde{g}(y):=\left\{\begin{array}{ll}
0 & \text { for }|y|<1 \\
(n-2) \operatorname{D} f\left(y^{*}\right) \frac{y^{*}}{\left|y^{*}\right|^{n}|y|^{2 n}} & \text { for }\left|y^{*}\right|<1
\end{array} .\right.
$$

When we change variables in the second and the third integral on the right hand side of (3.5) the first term can be grouped with the second. Then, for $i \in\{1,2, \ldots, n\}$ we can differentiate with respect to $x_{i}$ to get

$$
\partial_{i} h(x)-\partial_{i} f(x)=\partial_{i} \operatorname{div}(g * \Gamma)(x)+\int_{\mathbb{R}^{n}} \widetilde{g}(y) \partial_{i} \Gamma(x-y) \mathrm{d} \mathscr{L}^{n}(y) .
$$


Summing over $i \in\{1,2, \ldots, n\}$ it follows that

$$
\int_{\mathbf{B}_{1}^{n}}|\mathrm{D} h|^{p} \mathrm{~d} \mathscr{L}^{n} \leq C\left[\int_{\mathbf{B}_{1}^{n}}|\mathrm{D} f|^{p} \mathrm{~d} \mathscr{L}^{n}+\sum_{i, j=1}^{n} \mathrm{I}_{i, j}+\sum_{i=1}^{n} \mathrm{II}_{i}\right],
$$

where $C$ depends only on $n$ and $p$, and where we have set

$$
\begin{aligned}
\mathrm{I}_{i, j} & :=\int_{\mathbf{B}_{1}^{n}}\left|\partial_{i} \partial_{j}(\Gamma * g)\right|^{p} \mathrm{~d} \mathscr{L}^{n}, \\
\mathrm{II}_{i} & :=\int_{\mathbf{B}_{1}^{n}}\left|\int_{\mathbb{R}^{n}} \widetilde{g}(y) \partial_{i} \Gamma(x-y) \mathrm{d} \mathscr{L}^{n}(y)\right|^{p} \mathrm{~d} \mathscr{L}^{n}(x) .
\end{aligned}
$$

To estimate $\mathrm{I}_{i, j}$ we exploit the $\mathrm{L}^{p}$-estimates for the second derivatives of the Newton potential $g * \Gamma$ of $g$. Adapting the proof of [22, Theorem 9.9] to the vectorial case these estimates can — with moderate effort ${ }^{5}$ - be verified with an $N$-independent constant. Via these estimates, using the definition of $g$ and changing back to the original variable we get

$$
\begin{aligned}
\mathrm{I}_{i, j} & \leq C \int_{\mathbb{R}^{n}}|g|^{p} \mathrm{~d} \mathscr{L}^{n} \leq C\left[\int_{\mathbf{B}_{1}^{n}}|\mathrm{D} f|^{p} \mathrm{~d} \mathscr{L}^{n}+\int_{\mathbf{B}_{1}^{n}}\left(\frac{|\mathrm{D} f(y)|}{|y|^{n}\left|y^{*}\right|^{2 n}}\right)^{p} \frac{\mathrm{d} \mathscr{L}^{n}(y)}{|y|^{2 n}}\right] \\
& =C \int_{\mathbf{B}_{1}^{n}}|\mathrm{D} f(y)|^{p}\left(1+|y|^{p n-2 n}\right) \mathrm{d} \mathscr{L}^{n}(y) .
\end{aligned}
$$

In order to bound the non-singular terms $\mathrm{II}_{i}$ we rely on the fact that $\widetilde{g}$ vanishes outside $\mathbf{B}_{2}^{n}$, which in turn follows from the assumption $f \equiv 0$ on $\mathbf{B}_{\frac{1}{2}}^{n}$. Indeed, via the control $\left|\partial_{i} \Gamma(x-y)\right| \leq C|x-y|^{1-n}$, Hölder's inequality, Fubini's theorem, and the same arguments as before yield

$$
\begin{aligned}
\mathrm{II}_{i} & \leq C \int_{\mathbf{B}_{1}^{n}} \int_{\mathbf{B}_{2}^{n}}|\widetilde{g}(y)|^{p} \frac{\mathrm{d} \mathscr{L}^{n}(y)}{|x-y|^{n-1}}\left(\int_{\mathbf{B}_{2}^{n}} \frac{\mathrm{d} \mathscr{L}^{n}(y)}{|x-y|^{n-1}}\right)^{p-1} \mathrm{~d} \mathscr{L}^{n}(x) \\
& \leq C \int_{\mathbf{B}_{2}^{n}}|\widetilde{g}(y)|^{p} \int_{\mathbf{B}_{1}^{n}} \frac{\mathrm{d} \mathscr{L}^{n}(x)}{|x-y|^{n-1}} \mathrm{~d} \mathscr{L}^{n}(y) \leq C \int_{\mathbf{B}_{2}^{n}}|\widetilde{g}(y)|^{p} \mathrm{~d} \mathscr{L}^{n}(y) \\
& \leq C \int_{\mathbf{B}_{1}^{n}}\left(\frac{|\mathrm{D} f(y)|}{|y|^{n-1}\left|y^{*}\right|^{2 n}}\right)^{p} \frac{\mathrm{d} \mathscr{L}^{n}(y)}{|y|^{2 n}}=C \int_{\mathbf{B}_{1}^{n}}|\mathrm{D} f(y)|^{p}|y|^{p+p n-2 n} \mathrm{~d} \mathscr{L}^{n}(y) .
\end{aligned}
$$

When we combine (3.6), (3.7), and (3.8) and control the occurrences of $|\mathrm{D} f|^{p}$ by its ess sup, we arrive at the claim (3.3).

\footnotetext{
${ }^{5}$ We here rely only on the most classical and simplest $\mathrm{L}^{p}$-estimates as originally established by Calderon \& Zygmund [11]. The proof of these singular-integral estimates requires only the CalderonZygmund covering arguments and the Marcinkiewicz interpolation theorem (see again [22, Chapter 9] and compare also [24, Theorem 2.13] for a vector-valued version of the latter theorem). In particular, our whole approach does not rely on flattening-of-the-boundary arguments.
} 
Remark 3.2. Even though it will not play a role in our reasoning, we find it worthwhile to record that for $2 \leq p<\infty$ the exponents at $|y|$ in (3.7) and (3.8) are positive, and then the same approach (with a slightly modified reduction argument at the beginning of the proof) also yields a stronger form of (3.3), where $\sigma^{n} \sup _{\mathbf{B}_{\sigma}^{n}}|\mathrm{D} f|^{p}$ is replaced by $\int_{\mathbf{B}_{\sigma}^{n}}|\mathrm{D} f|^{p} \mathrm{~d} \mathscr{L}^{n}$.

In the sequel, we call a function $h \in \mathrm{L}^{2}\left(\mathbf{B}_{r}^{n} ; \mathcal{H}\right)$ harmonic if $\langle h, a\rangle$ is harmonic for all vectors $a \in \mathcal{H}$. Thanks to the codimension-free estimates of the Lemma 3.1 we can easily provide the following existence and uniqueness result.

Theorem 3.3. For every Lipschitz function $f: \mathbf{B}_{\sigma}^{n} \rightarrow \mathcal{H}$ there exists a unique harmonic $h \in \mathrm{C}\left(\overline{\mathbf{B}_{\sigma}^{n}} ; \mathcal{H}\right)$ with $h=f$ on $\partial \mathbf{B}_{\sigma}^{n}$. It satisfies

$$
|\mathrm{D} h(y)-\mathrm{D} h(x)|^{2} \leq C \frac{|y-x|^{2}}{(\sigma-\eta)^{n+2}} \int_{\mathbf{B}_{\sigma}^{n}}|\mathrm{D} h|^{2} \mathrm{~d} \mathscr{L}^{n} \quad \text { for } 0<\eta<\sigma \text { and } x, y \in \mathbf{B}_{\eta}^{n},
$$

where $C$ depends only on $n$, and

$$
\int_{\mathbf{B}_{\sigma}^{n}}|\mathrm{D} h|^{p} \mathrm{~d} \mathscr{L}^{n} \leq C \sigma^{n} \underset{\mathbf{B}_{\sigma}^{n}}{\operatorname{ess} \sup }|\mathrm{D} f|^{p},
$$

where $C$ depends only on $n$ and $p$.

Proof. Uniqueness can be easily achieved arguing component-wise, so let us focus on existence. Let $\left(e_{n}\right)_{n \in \mathbb{N}}$ be an orthonormal basis of $\mathcal{H}$, and denote by $f_{N}$ the Lipschitz function which is the composition of $f$ with the projection on $\operatorname{Span}\left(e_{1}, \ldots, e_{N}\right)$. Since $f\left(\overline{\mathbf{B}_{\sigma}^{n}}\right)$ is compact in $\mathcal{H}$, for $N \rightarrow \infty$ we clearly have $f_{N} \rightarrow f$ uniformly in $\overline{\mathbf{B}_{\sigma}^{n}}$. Furthermore, $\left|\mathrm{D} f_{N}\right| \leq|\mathrm{D} f|$ holds $\mathscr{L}^{n}$-a. e., and the $\mathrm{D} f_{N}$ converge to $\mathrm{D} f$ in the Hilbert space $\mathrm{L}^{2}\left(\mathbf{B}_{\sigma}, \mathrm{L}\right)$, where $\mathrm{L}$ denotes the space of linear maps from $\mathbb{R}^{n}$ to $\mathcal{H}$.

We let $h_{N} \in \mathrm{C}\left(\overline{\mathbf{B}_{\sigma}^{n}}, \mathcal{H}\right)$ be the harmonic functions taking values in $\operatorname{Span}\left(e_{1}, \ldots, e_{N}\right)$ and coinciding with $f_{N}$ on $\partial \mathbf{B}_{\sigma}^{n}$. In particular, we then have $\int_{\mathbf{B}_{\sigma}^{n}}\left|\mathrm{D} h_{N}\right|^{2} \mathrm{~d} \mathscr{L}^{n} \leq$ $\int_{\mathbf{B}_{\sigma}^{n}}\left|\mathrm{D} f_{N}\right|^{2} \mathrm{~d} \mathscr{L}^{n}$, and moreover we can apply Lemma 3.1: the corresponding estimates (3.1) and (3.2) show that the maps $h_{N}$ and $\mathrm{D} h_{N}$ are locally equi-Lipschitz in $\mathbf{B}_{\sigma}^{n}$, and moreover

$$
\int_{\mathbf{B}_{\sigma}^{n}}\left|\mathrm{D} h_{N}\right|^{p} \mathrm{~d} \mathscr{L}^{n} \leq C \sigma^{n} \underset{\mathbf{B}_{\sigma}^{n}}{\operatorname{ess} \sup }|\mathrm{D} f|^{p}
$$

with $C$ depending only on $n$ and $p$.

Given any unit vector $a \in \mathcal{H}$ we can apply the maximum principle to $\left\langle h_{N}-h_{M}, a\right\rangle$ : we thus achieve $\max _{\overline{\mathbf{B}_{\sigma}^{n}}}\left|h_{N}-h_{M}\right| \leq \sup _{|a|=1} \max _{\overline{\mathbf{B}_{\sigma}^{n}}}\left\langle h_{N}-h_{M}, a\right\rangle \leq \max _{\partial \mathbf{B}_{\sigma}^{n}}\left|f_{N}-f_{M}\right| \rightarrow$ 
0 . Hence, the $h_{N}$ converge uniformly to a function $h \in \mathrm{C}\left(\overline{\mathbf{B}_{\sigma}^{n}}, \mathcal{H}\right)$. By the Dirichlet principle we have

$$
\int_{\mathbf{B}_{\sigma}^{n}}\left|\mathrm{D} h_{N}-\mathrm{D} h_{M}\right|^{2} \mathrm{~d} \mathscr{L}^{n} \leq \int_{\mathbf{B}_{\sigma}^{n}}\left|\mathrm{D} f_{N}-\mathrm{D} f_{M}\right|^{2} \mathrm{~d} \mathscr{L}^{n}
$$

for $N, M \in \mathbb{N}$. Since $\left(\mathrm{D} f_{N}\right)_{N \in \mathbb{N}}$ is a Cauchy sequence in $\mathrm{L}^{2}\left(\mathbf{B}_{\sigma}^{n}, \mathrm{~L}\right)$, so is $\left(\mathrm{D} h_{N}\right)_{N \in \mathbb{N}}$, which hence converges to a limit $\Upsilon$. Obviously $\langle h, a\rangle=\lim _{N \rightarrow \infty}\left\langle h_{N}, a\right\rangle$ is harmonic, and so is $h$ (by definition). Moreover, $h$ is locally Lipschitz continuous and hence a. e. differentiable. Using the convergence $\left\langle h_{N}, a\right\rangle \rightarrow\langle h, a\rangle$ and the harmonicity, we easily conclude that $\Upsilon=\mathrm{D} h$; thus, we have $\mathrm{D} h_{N} \rightarrow \mathrm{D} h$ in $\mathrm{L}^{2}\left(\mathbf{B}_{\sigma}^{n}, \mathrm{~L}\right)$, and (3.9) follows. Combining the preceding pieces of information with the local uniform regularity of $\mathrm{D} h_{N}$, we obtain that $\mathrm{D} h$ has a continuous representative in $\mathbf{B}_{\sigma}^{n}$, and that $\mathrm{D} h_{N} \rightarrow \mathrm{D} h$ locally uniformly in $\mathbf{B}_{\sigma}^{n}$. This provides at once (3.10) and (3.11) by a passage to the limit. In connection with (3.12), we use (3.13) to obtain

$$
\int_{B_{\eta}^{n}}|\mathrm{D} h|^{p} \mathrm{~d} \mathscr{L}^{n} \leq C \sigma^{n} \underset{B_{\sigma}^{n}}{\operatorname{esssup}}|\mathrm{D} f|^{p},
$$

with $\eta<\sigma$, and then let $\eta \nearrow \sigma$.

\section{THE $\varepsilon$-REGULARITY THEOREM}

In this section we state the main $\varepsilon$-regularity theorem for mass-minimizing currents. The basic assumption that allows to initiate the regularization process is stated below.

Assumption 4.1. We assume that $T \in \mathbf{I}_{n}(\mathcal{H})$ is locally mass-minimizing in $z_{0}+\mathbf{C}_{r}^{\pi}$ with

$$
\begin{gathered}
(\partial T)\left\llcorner\left(z_{0}+\mathbf{C}_{r}^{\pi}\right) \equiv 0,\right. \\
\left(\mathrm{p}^{\pi}\right)_{\sharp}\left(T\left\llcorner\left(z_{0}+\mathbf{C}_{r}^{\pi}\right)\right)=m \llbracket z_{0}+\mathbf{B}_{r}^{\pi} \rrbracket,\right. \\
\Theta^{n}(\|T\|, z) \geq m \text { for }\|T\|-a . \text { e. } z \in z_{0}+\mathbf{C}_{r}^{\pi}, \\
\mathbf{E}\left(T, z_{0}, r, \pi\right) \leq \varepsilon
\end{gathered}
$$

for positive parameters $r, \varepsilon$, some $n$-plane $\pi$ in $\mathcal{H}, z_{0} \in \operatorname{Span} \pi$, and $m \in \mathbb{N}$.

Indeed, when these conditions are satisfied, we will say that Assumption 4.1 (with multiplicity $m$ ) holds for $T$ on $z_{0}+\mathbf{C}_{r}^{\pi}$ up to $\varepsilon$. We will occasionally refer to the radius $r$ as the scale of the assumption.

By the constancy theorem, (4.1) automatically implies $\left(\mathrm{p}^{\pi}\right)_{\sharp}\left(T \mathrm{~L}\left(z_{0}+\mathbf{C}_{r}^{\pi}\right)\right)=m^{\prime} \llbracket z_{0}+\mathbf{B}_{r}^{\pi} \rrbracket$ for some integer $m^{\prime}$. Therefore, - apart from the requirement that $m^{\prime}$ should be positive, which can always be achieved replacing $T$ by $-T$ - the main point in imposing (4.2) is to guarantee that $m=m^{\prime}$ (i. e. that $m^{\prime}$ equals the lower bound $m$ in (4.3)).

In the sequel we permanently fix the multiplicity $m \in \mathbb{N}$, and in particular when referring to Assumption 4.1 we always mean the statement with this fixed multiplicity.

We can now state our $\varepsilon$-regularity theorem. 
Theorem 4.2 ( $\varepsilon$-regularity theorem for mass-minimizing currents). There exists a positive constant $\varepsilon_{*}$, depending only on $n, m \in \mathbb{N}$, with the following property: whenever Assumption 4.1 holds for a current $T$ on $\mathbf{C}_{\varrho}^{\pi}$ up to $\varepsilon \leq \varepsilon_{*}$, then there exists a function $f: \mathbf{B}_{r / 2}^{\pi} \rightarrow(\operatorname{Span} \pi)^{\perp}$, which is $\mathrm{C}^{1, \alpha}$ for all $\alpha<1$ and such that $T\left\llcorner\mathbf{C}_{r / 2}^{\pi}\right.$ is represented by the graph of $f$ with multiplicity $m$, in more precise terms

$$
T\left\llcorner\mathbf{C}_{r / 2}^{\pi}=m \llbracket \text { Graph } f \rrbracket .\right.
$$

The proof of Theorem 4.2 will be carried out in the following two sections and will be finalized at the end of Section 6 .

\section{VerticAl SEPARATION AND LipsCHITZ APPROXIMATION}

As in [32], we rely on a vertical separation lemma, which is often called Federer's height bound, although a result of this type appeared first in [2]. The proof in our setting is widely analogous to the one of [32, Lemma 2], which in turn resembles the argument of Federer [20, Lemma 5.3.4], but nevertheless we prefer to carry out the details.

Lemma 5.1 (Vertical separation). There exist $\varepsilon_{0}=\varepsilon_{0}(n, m)$ and $\lambda_{0}=\lambda_{0}(n, m)$ with the following property. Whenever Assumption 4.1 holds for $T$ on $z_{0}+\mathbf{C}_{r}^{\pi}$ up to $\varepsilon \leq \varepsilon_{0}$ for some $n$-plane $\pi$, then we have

$$
\left|\mathrm{q}^{\pi}\left(z_{1}\right)-\mathrm{q}^{\pi}\left(z_{2}\right)\right| \leq \lambda_{0} r \mathbf{E}^{1 /(2 n)}\left(T, z_{0}, r, \pi\right) \quad \forall z_{1}, z_{2} \in \operatorname{spt} T \cap\left(z_{0}+\mathbf{C}_{r / 2}^{\pi}\right) .
$$

Proof. By scaling we can assume $z_{0}=0$ and $r=1$. To simplify the notation we set $\mathbf{E}:=\mathbf{E}(T, 0,1, \pi)$, and we write $\mathrm{p}=\mathrm{p}^{\pi}, \mathrm{q}=\mathrm{q}^{\pi}$ for the orthogonal projections onto Span $\pi$ and its orthogonal complement, respectively. We will assume in the following that $\varepsilon_{0}$ is chosen sufficiently small so that three smallness conditions, needed during the proof, hold.

We fix a unit vector $a \in(\operatorname{Span} \pi)^{\perp}$ and for $s_{1}, s_{2} \in[-\infty,+\infty]$ with $s_{1} \leq s_{2}$ we define

$$
S\left(s_{1}, s_{2}\right):=\left\{z \in \mathbf{C}_{1}^{\pi}:\langle z, a\rangle \in\left(s_{1}, s_{2}\right)\right\} .
$$

We denote by $\bar{s}$ a median value for the function $s \mapsto\|T\|(S(-\infty, s))$, namely

$$
\|T\|(S(-\infty, \bar{s})) \leq \frac{1}{2}\|T\|\left(\mathbf{C}_{1}^{\pi}\right) \quad \text { and } \quad\|T\|(S(\bar{s},+\infty)) \leq \frac{1}{2}\|T\|\left(\mathbf{C}_{1}^{\pi}\right)
$$

and we denote by $s_{*}$ be the supremum of all $s>\bar{s}$ such that $\|T\|(S(s,+\infty)) \geq \sqrt{\mathbf{E}}$. If no such $s$ exists we set $s_{*}=\bar{s}$. We also impose the smallness condition $\varepsilon_{0} \leq 1$, so that $\|T\|(S(s,+\infty)) \geq \sqrt{\mathbf{E}} \geq \mathbf{E}$ for $s \in\left[\bar{s}, s_{*}\right)$.

We recall that every $n$-dimensional integral current $R$ with support in Span $\pi$ can be canonically identified with an integer-valued function of bounded variation $f$. We shall use the following Sobolev-type inequality for such integer-valued BV functions, which can be derived from the isoperimetric inequality (see for instance [20, 5.3.2]):

$$
\left(\int_{\mathbf{B}_{1}^{\pi}}|f| d \mathscr{L}^{n}\right)^{1-1 / n} \leq \xi_{n}|\mathrm{D} f|\left(\mathbf{B}_{1}^{\pi}\right) \quad \text { if } \quad \mathscr{L}^{n}\left(\mathbf{B}_{1}^{\pi} \cap\{f=0\}\right) \geq \frac{\omega_{n}}{4} .
$$


Here $|\mathrm{D} f|$ is the total variation of the distributional derivative of $f$ and $\xi_{n}$ is a constant which depends on $n$. In terms of the current $R$ associated to $f$, the inequality reads

$$
\left(\|R\|\left(\mathbf{B}_{1}^{\pi}\right)\right)^{1-1 / n} \leq \xi_{n}\|\partial R\|\left(\mathbf{B}_{1}^{\pi}\right) \quad \text { if } \quad \mathscr{L}^{n}\left(\mathbf{B}_{1}^{\pi} \cap\left\{\Theta^{n}(\|R\|, \cdot)=0\right\}\right) \geq \frac{\omega_{n}}{4} .
$$

For a. e. $s \in \mathbb{R}$, we introduce the integral currents $T_{s}=T\left\llcorner S(s,+\infty)\right.$ and $R_{s}:=$ $\mathrm{p}_{\sharp} T_{s}$, and we now aim to apply (5.3) to $R_{s}$. Since (2.18) and (4.2) yield $\|T\|\left(\mathbf{C}_{1}^{\pi}\right) \leq$ $m \omega_{n}+\mathbf{E} / 2 \leq(3 m / 2) \omega_{n}$ as soon as $\varepsilon_{0} \leq m \omega_{n}$, we get

$$
\|T\|(S(\bar{s},+\infty)) \leq \frac{3}{4} m \omega_{n} .
$$

For all $s \geq \bar{s}$ it holds

$$
\begin{aligned}
\mathscr{L}^{n}\left(\mathbf{B}_{1}^{\pi} \cap\left\{\Theta^{n}\left(\left\|R_{s}\right\|, \cdot\right)>0\right\}\right) & \leq \mathscr{H}^{n}\left(\mathbf{C}_{1}^{\pi} \cap\left\{\Theta^{n}\left(\left\|T_{s}\right\|, \cdot\right)>0\right\}\right) \\
& =\mathscr{H}^{n}\left(\mathbf{C}_{1}^{\pi} \cap\left\{\Theta^{n}\left(\left\|T_{s}\right\|, \cdot\right) \geq m\right\}\right) \\
& \leq \frac{1}{m}\|T\|(S(s,+\infty)) \leq \frac{1}{m}\|T\|(S(\bar{s},+\infty)) \leq \frac{3}{4} \omega_{n} .
\end{aligned}
$$

In the above chain of inequalities, the first follows from $\left\|R_{s}\right\|\left(\mathbf{B}_{1}^{\pi} \backslash \mathrm{p}\left(\operatorname{spt}\left(T_{s}\right)\right)\right)=0$ and $\operatorname{spt}(T)=\left\{\Theta^{n}\left(\left\|T_{s}\right\|, \cdot\right)>0\right\}$, the subsequent equality comes from (4.3), and the last inequality is obtained from (5.4). Thus, we can indeed apply (5.3) and use the identity $\partial R_{s}=\mathrm{p}_{\sharp} \partial T_{s}$ to get

$$
\left(\mathbf{M}\left(R_{s}\right)\right)^{1-1 / n} \leq \xi_{n}\left\|\partial R_{s}\right\|\left(\mathbf{B}_{1}^{\pi}\right) \leq \xi_{n}\left\|\partial T_{s}\right\|\left(\mathbf{C}_{1}^{\pi}\right) \text { for a. e. } s \geq \bar{s} .
$$

Now we can apply $(2.18)$ to $T_{s}$ to get

$$
\mathbf{M}\left(T_{s}\right) \leq \mathbf{M}\left(R_{s}\right)+\frac{1}{2} \int_{S(s,+\infty)}|\vec{T}-\pi|^{2} \mathrm{~d}\|T\| \leq \mathbf{M}\left(R_{s}\right)+\frac{1}{2} \mathbf{E}
$$

for $s \geq \bar{s}$. If $s<s_{*}$ it follows that $\mathbf{E} \leq \mathbf{M}\left(R_{s}\right)+\mathbf{E} / 2$, whence $\mathbf{E} \leq 2 \mathbf{M}\left(R_{s}\right)$. Coming back to the previous inequality we get

$$
\mathbf{M}\left(T_{s}\right) \leq 2 \mathbf{M}\left(R_{s}\right) \quad \forall s \in\left[\bar{s}, s_{*}\right) .
$$

This inequality, combined with (5.5), gives

$$
\left(\mathbf{M}\left(T_{s}\right)\right)^{1-1 / n} \leq 2^{1-1 / n} \xi_{n}\left\|\partial T_{s}\right\|\left(\mathbf{C}_{1}^{\pi}\right) \quad \text { for a. e. } s \in\left(\bar{s}, s_{*}\right) .
$$

Now we integrate on $\left(\bar{s}, s_{*}\right)$ and use $(2.8)$ with the slicing function $\psi(z):=\langle z, a\rangle$ to get

$$
\begin{aligned}
\left(s_{*}-\bar{s}\right)\left(\inf _{s \in\left(\bar{s}, s_{*}\right)} \mathbf{M}\left(T_{s}\right)\right)^{1-1 / n} & \leq \int_{\bar{s}}^{s_{*}}\left(\mathbf{M}\left(T_{s}\right)\right)^{1-1 / n} \mathrm{~d} s \leq 2 \xi_{n} \int_{\bar{s}}^{s_{*}}\left\|\partial T_{s}\right\|\left(\mathbf{C}_{1}^{\pi}\right) \mathrm{d} s \\
& =2 \xi_{n} \| T\left\llcorner\mathrm{~d} \psi \|\left(S\left(\bar{s}, s_{*}\right)\right)=2 \xi_{n} \int_{S\left(\bar{s}, s_{*}\right)} \mid \vec{T}\llcorner a \mid \mathrm{d}\|T\|,\right.
\end{aligned}
$$

where $\vec{T}\left\llcorner a \in \Lambda_{n-1}(\mathcal{H})\right.$ is characterized by $\langle\vec{T}\llcorner a, \eta\rangle=\langle\vec{T}, a \wedge \eta\rangle$ for any $\eta \in$ $\Lambda_{n-1}(\mathcal{H})$. Since $a$ is orthogonal to Span $\pi$, using an orthonormal basis including $a$, it 
is easy to check that ${ }^{6} \mid \vec{T}\left\llcorner\left. a\right|^{2} \leq 1-\langle\vec{T}, \pi\rangle^{2} \leq|\vec{T}-\pi|^{2}\right.$. This last estimate, combined with the Hölder's inequality on the right hand side of (5.6) and with the choice of $s_{*}$ on the left hand side, yields $\left(s_{*}-\bar{s}\right) \sqrt{\mathbf{E}}^{1-1 / n} \leq 2 \xi_{n} \sqrt{\mathbf{E}} \sqrt{3 m \omega_{n} / 4}$, so that

$$
\left(s_{*}-\bar{s}\right) \leq \xi_{n} \sqrt{3 m \omega_{n}} \sqrt{\mathbf{E}}^{1 / n} .
$$

Assume now that there is $z \in \operatorname{spt} T \cap \mathbf{C}_{1 / 2}^{\pi}$ with $\langle z, a\rangle>s_{*}$ and let $\delta \in\left(0,\langle z, a\rangle-s_{*}\right)$, $\delta_{*}=\min \{\delta, 1 / 2\}$. Since the ball $z+\mathbf{B}_{\delta_{*}}$ is contained in $S(\langle z, a\rangle-\delta,+\infty)$, the lower mass bound (2.20) and the inequality $\langle z, a\rangle-\delta>s_{*}$ give

$$
\omega_{n} \delta_{*}^{n} \leq\|T\|(S(\langle z, a\rangle-\delta,+\infty)) \leq \sqrt{\mathbf{E}} .
$$

Since $\mathbf{E} \leq \varepsilon \leq \varepsilon_{0}$, if we impose the smallness condition $\left(\sqrt{\varepsilon_{0}} / \omega_{n}\right)^{1 / n} \leq 1 / 2$ we get $\delta_{*}=\delta$, so that we can let $\delta \uparrow\langle z, a\rangle-s_{*}$ to get

$$
\langle z, a\rangle-s_{*} \leq \frac{1}{\omega_{n}^{1 / n}} \sqrt{\mathbf{E}}^{1 / n} .
$$

This inequality can be combined with (5.7) to obtain

$$
\sup _{z \in \operatorname{spt} T \cap \mathbf{C}_{1 / 2}^{\pi}}\langle z, a\rangle-\bar{s} \leq \frac{\lambda_{0}}{2} \sqrt{\mathbf{E}}^{1 / n} .
$$

for some $\lambda_{0}=\lambda_{0}(n, m)$. A similar argument gives

$$
\inf _{z \in \operatorname{spt} T \cap \mathbf{C}_{1 / 2}^{\pi}}\langle z, a\rangle-\bar{s} \geq-\frac{\lambda_{0}}{2} \sqrt{\mathbf{E}}^{1 / n}
$$

and, since $a \in(\operatorname{Span} \pi)^{\perp}$ is arbitrary, the conclusion follows.

Before turning to the main estimates of this section, we record that Lemma 5.1 implies some inclusions of tilted cylinders, which will be useful later on.

Lemma 5.2 (tilting of cylinders). Suppose that we have $0 \in \operatorname{spt} T$ and that Assumption 4.1 holds for $T$ on $\mathbf{C}_{r}^{\pi}$ up to $\varepsilon \leq \lambda_{0}^{-2 n}$, where $\lambda_{0}$ is the constant from Lemma 5.1. Then, for $T_{r / 2}:=T\left\llcorner\mathbf{C}_{r / 2}^{\pi}\right.$, all $\tau \in\left(0, \frac{1}{4}\right]$, and all n-planes $\pi_{1}$ and $\pi_{2}$ in $\mathcal{H}$ we have

$$
\left|\pi_{2}-\pi_{1}\right| \leq \frac{\tau}{12 n} \Longrightarrow \mathbf{C}_{\tau r}^{\pi_{1}} \cap \operatorname{spt} T_{r / 2} \subset \mathbf{C}_{2 \tau r}^{\pi_{2}}
$$

Proof. We consider some $z \in \mathbf{C}_{\tau r}^{\pi_{1}} \cap \operatorname{spt} T_{r / 2}$. Then by Lemma 5.1, the assumption $0 \in \operatorname{spt} T$, and (4.4) with $\varepsilon \leq \lambda_{0}^{-2 n}$, we have $\left|\mathrm{q}^{\pi}(z)\right| \leq \lambda_{0} r \mathbf{E}^{1 /(2 n)}(T, 0, r, \pi) \leq r$. Together with $\left|\mathrm{p}^{\pi}(z)\right| \leq r / 2$ we find $|z| \leq 3 r / 2$ and via Lemma 2.2 we obtain

$$
\left|\mathrm{p}^{\pi_{2}}(z)\right| \leq\left|\mathrm{p}^{\pi_{1}}(z)\right|+8 n\left|\pi_{2}-\pi_{1}\right||z| \leq \tau r+12 n\left|\pi_{2}-\pi_{1}\right| r .
$$

Whenever $\left|\pi_{2}-\pi_{1}\right| \leq \tau /(12 n)$ holds, we thus get $\left|\mathrm{p}^{\pi_{2}}(z)\right| \leq 2 \tau r$ and $z \in \mathbf{C}_{2 \tau r}^{\pi_{2}}$.

\footnotetext{
${ }^{6}$ Indeed, writing as usual $\vec{T}=\bigwedge_{i} T_{i}$, one verifies the formulas $\vec{T}\left\llcorner a=\sum_{j=1}^{n}(-1)^{j-1}\left\langle T_{j}, a\right\rangle \bigwedge_{i \neq j} T_{i}\right.$ and $1=|\vec{T}|^{2} \geq\left|\bigwedge_{i} \mathrm{p}^{\pi}\left(T_{i}\right)\right|^{2}+\left|\bigwedge_{i}\left\langle T_{i}, a\right\rangle a\right|^{2}=\langle\vec{T}, \pi\rangle^{2}+\mid \vec{T}\left\llcorner\left. a\right|^{2}\right.$.
} 
Next, we will construct a Lipschitz graph approximating $T$ in the sense of the following lemma.

Lemma 5.3 (Lipschitz approximation). Let (4.1), (4.2), and (4.3) hold for $T$ on $\mathbf{C}_{r}^{\pi}$. Then for every $\lambda \in(0,1]$ there exists a Lipschitz function $f: \mathbf{B}_{r / 2}^{\pi} \rightarrow(\operatorname{Span} \pi)^{\perp}$ with

$$
\operatorname{Lip}(f) \leq \lambda
$$

such that $T^{\mathrm{g}}:=m \llbracket$ Graph $f \rrbracket \in \mathbf{I}_{n}(\mathcal{H})$ approximates $T$ in $\mathbf{C}_{r / 2}^{\pi}$ in the sense of

$$
\begin{gathered}
T^{\mathrm{g}}\left\llcorner\left(\mathrm{p}^{\pi}\right)^{-1}\left(G_{\lambda}\right)=T\left\llcorner\left(\mathrm{p}^{\pi}\right)^{-1}\left(G_{\lambda}\right),\right.\right. \\
\left\|T^{\mathrm{g}}-T\right\|\left(\mathbf{C}_{r / 2}^{\pi}\right) \leq C \lambda^{-2} r^{n} \mathbf{E}(T, 0, r, \pi),
\end{gathered}
$$

where the closed subset $G_{\lambda}$ of $\mathbf{B}_{r / 2}^{\pi}$ will be specified in Lemma 5.5 below, and where $C$ depends only on $m$ and $n$.

Finally, if the full Assumption 4.1 holds for $T$ on $\mathbf{C}^{\pi}$ up to $\varepsilon \leq \varepsilon_{0}$, then we additionally have the oscillation bound

$$
\sup _{\mathbf{B}_{r / 2}^{\pi}}|f-f(0)| \leq \lambda_{0} r \mathbf{E}^{1 /(2 n)}(T, 0, r, \pi),
$$

where $\varepsilon_{0}$ and $\lambda_{0}$ are the constants of Lemma 5.1 .

In principle, the approximation result of Lemma 5.3 is well-known, and even in our case of an infinite-dimensional ambient space we could follow, for instance, the proof of [32, Lemma 3], which is based on vertical separation. We prefer however to involve an alternative and more recent idea from [15], which relies on a refined version of the Jerrard-Soner BV estimate [26, 27] and on well-known inequalities for maximal functions. This approach leads to two slight improvements in Lemma 5.3, when compared to the more classical statements: on the one hand, we obtain the optimal power $\lambda^{-2}$ in (5.9) (while [32, Lemma 3] contains a factor $\lambda^{-2 n}$ instead); on the other hand, in order to obtain (5.8) and (5.9) we only assume (4.1), (4.2), and (4.3), where in particular the last hypothesis rules out branching phenomena. In contrast, the full strength of Assumption 4.1 is only needed for Lemma 5.1 and the corresponding bound (5.10).

For the remainder of this section we abbreviate again $\mathrm{p}=\mathrm{p}^{\pi}, \mathrm{q}=\mathrm{q}^{\pi}$, and we set $T_{x}:=\mathrm{q}_{\sharp}\langle T, \mathrm{p}, x\rangle$, where $\langle T, \mathrm{p}, x\rangle$ are the 0 -dimensional slices of the current $T$ with respect to the slicing map $\mathrm{p}$ according to [5, Theorem 5.6]. Moreover, given any $\psi \in \operatorname{Lip}_{\mathrm{b}}\left((\operatorname{Span} \pi)^{\perp}\right)$ we introduce the notation

$$
\Phi_{\psi}(x):=T_{x}(\psi) \quad \text { for } \mathscr{L}^{n} \text {-a. e. } x \in \mathbf{B}_{1}^{\pi} .
$$

We next provide the announced version of the BV estimate used in [5] to estimate the BV norm of $\Phi_{\psi}$. To keep our presentation elementary, we state it without appealing to the general theory of metric-space valued BV maps developed in [3]. 
Lemma 5.4 (BV estimate). Suppose that $T \in \mathbf{I}_{n}(\mathcal{H})$ satisfies (4.1) and (4.2) on $\mathbf{C}_{1}^{\pi}$ (i. e. with $z_{0}=0$ and $\left.r=1\right)$. For every bounded $\psi \in \mathrm{C}^{1}\left((\operatorname{Span} \pi)^{\perp}\right)$ with $\operatorname{Lip}(\psi) \leq 1$, we then have $\Phi_{\psi} \in \mathrm{BV}\left(\mathbf{B}_{1}^{\pi}\right)$, and on every Borel subset $A \subset \mathbf{B}_{1}^{\pi}$ there holds

$$
\left(\left|\mathrm{D} \Phi_{\psi}\right|(A)\right)^{2} \leq 2 n\left(\|T\|\left(\mathrm{p}^{-1}(A)\right)-m \mathscr{L}^{n}(A)\right)\|T\|\left(\mathrm{p}^{-1}(A)\right) .
$$

Proof. It suffices to prove (5.11) for an open subset $A$ of $\mathbf{B}_{1}^{\pi}$. For such $A$ we recall from $[4$, Proposition 3.6] that

$$
\left|\mathrm{D} \Phi_{\psi}\right|(A)=\sup \left\{\int_{A} \Phi_{\psi} \operatorname{div} \varphi \mathrm{d} \mathscr{L}^{n}: \varphi \in \mathrm{C}_{\mathrm{c}}^{1}(A, A), \sup _{A}|\varphi| \leq 1\right\} .
$$

Here, writing $\pi=\bigwedge_{i=1}^{n} \pi_{i}$, with an orthonormal base $\pi_{1}, \pi_{2}, \ldots, \pi_{n}$ for $\operatorname{Span} \pi$, and $\varphi=\sum_{i=1}^{n} \varphi_{i} \pi_{i}$, the divergence is computed as $\operatorname{div} \varphi(x):=\sum_{i=1}^{n} \mathrm{D} \varphi_{i}(x) \pi_{i}$. Next we extend $\varphi$ from Span $\pi$ to all of $\mathcal{H}$ by $\varphi(z):=\varphi(\mathrm{p}(z))$ and $\psi$ from (Span $\pi)^{\perp}$ to all of $\mathcal{H}$ by $\psi(z):=\psi(\mathrm{q}(z))$, we define $\mathrm{p}_{i}^{\pi} \in \operatorname{Lip}(\mathcal{H})$ by $\mathrm{p}_{i}^{\pi}(z):=\left\langle\pi_{i}, z\right\rangle$, and we abbreviate

$$
\Xi:=\sum_{j=1}^{n}(-1)^{j-1} \varphi_{j} \bigwedge_{\substack{i=1 \\ i \neq j}}^{n} \mathrm{dp}_{i}^{\pi}, \quad \text { and correspondingly } \mathrm{d} \Xi:=(\operatorname{div} \varphi) \bigwedge_{i=1}^{n} \mathrm{dp}_{i}^{\pi}
$$

As $\varphi$ is supported in $\mathbf{C}_{1}^{\pi}$ and $(\partial T)\left\llcorner\mathbf{C}_{1}^{\pi}\right.$ vanishes by (4.1), the product and chain rules from [5, Theorem 3.5] give

$$
T(\psi \mathrm{d} \Xi)=-T(\mathrm{~d} \psi \wedge \Xi)
$$

(where the right-hand side can be understood with the help of (2.15)). Using the property of the slice map (see formula (5.7) in [5]), we arrive at

$$
\begin{aligned}
\int_{A} \Phi_{\psi} \operatorname{div} \varphi \mathrm{d} \mathscr{L}^{n} & =\int_{\mathbf{B}_{1}^{\pi}} T_{x}(\psi) \operatorname{div} \varphi(x) \mathrm{d} \mathscr{L}^{n}(x)=T(\psi \mathrm{d} \Xi) \\
& =-T(\mathrm{~d} \psi \wedge \Xi))=\sum_{j=1}^{n}(-1)^{j} \int_{\mathbf{C}_{1}^{\pi}} \varphi_{j}\left\langle\mathrm{D} \psi \wedge \bigwedge_{\substack{i=1 \\
i \neq j}}^{n} \pi_{i}, \vec{T}\right\rangle \mathrm{d}\|T\|
\end{aligned}
$$

where the gradient $\mathrm{D} \psi$ takes values in $(\operatorname{Span} \pi)^{\perp}$ and thus satisfies $\left\langle\mathrm{D} \psi \wedge \bigwedge_{i \neq j} \pi_{i}, \pi\right\rangle \equiv$ 0 . Therefore, we can also write

$$
\int_{A} \Phi_{\psi} \operatorname{div} \varphi \mathrm{d} \mathscr{L}^{n}=\sum_{j=1}^{n}(-1)^{j} \int_{\mathbf{C}_{1}^{\pi}} \varphi_{j}\left\langle\mathrm{D} \psi \wedge \bigwedge_{\substack{i=1 \\ i \neq j}}^{n} \pi_{i}, \vec{T}-\langle\vec{T}, \pi\rangle \pi\right\rangle \mathrm{d}\|T\|,
$$

and, since $\varphi$ is supported in $\mathrm{p}^{-1}(A)$, we conclude

$$
\begin{aligned}
\left(\int_{A} \Phi_{\psi} \operatorname{div} \varphi \mathrm{d} \mathscr{L}^{n}\right)^{2} & \leq n^{2} \sup _{\mathbf{C}_{1}^{\pi}}(|\varphi||\mathrm{D} \psi|)^{2}\left(\int_{\mathrm{p}^{-1}(A)}|\vec{T}-\langle\vec{T}, \pi\rangle \pi| \mathrm{d}\|T\|\right)^{2} \\
& \leq n^{2}\|T\|\left(\mathrm{p}^{-1}(A)\right) \int_{\mathrm{p}^{-1}(A)}|\vec{T}-\langle\vec{T}, \pi\rangle \pi|^{2} \mathrm{~d}\|T\| .
\end{aligned}
$$


However, (4.2) and the considerations leading to (2.18) yield

$$
\int_{\mathrm{p}^{-1}(A)}|\vec{T}-\langle\vec{T}, \pi\rangle \pi|^{2} \mathrm{~d}\|T\|=\|T\|\left(\mathrm{p}^{-1}(A)\right)-m \mathscr{L}^{n}(A) .
$$

Combining the last two formulas and recalling (5.12), we arrive at the claim (5.11).

Lemma 5.5 (Lipschitz estimate for good points). There exists a positive constant $\gamma \in\left(0, \omega_{n}\right]$, depending only on $n$ and $m$, with the following property. If $T \in \mathbf{I}_{n}(\mathcal{H})$ satisfies (4.1) and (4.2) on $\mathbf{C}_{r}^{\pi}$, then for all $\lambda \in(0,1]$ the set

$$
G_{\lambda}:=\left\{x \in \mathbf{B}_{r / 2}^{\pi}: \mathbf{E}(T, \widetilde{x}, \varrho, \pi) \leq \gamma \lambda^{2} \quad \forall(\widetilde{x}, \varrho) \in \mathbf{B}_{r / 2}^{\pi} \times(0, r / 2) \text { with } x \in \widetilde{x}+\mathbf{B}_{\varrho}^{\pi}\right\}
$$

of good points satisfies

$$
\begin{gathered}
\mathscr{L}^{n}\left(\mathbf{B}_{r / 2}^{\pi} \backslash G_{\lambda}\right)+\|T\|\left(\mathrm{p}^{-1}\left(\mathbf{B}_{r / 2}^{\pi} \backslash G_{\lambda}\right)\right) \leq \frac{C r^{n}}{\gamma \lambda^{2}} \mathbf{E}(T, 0, r, \pi), \\
\|T\|\left(\mathrm{p}^{-1}(N)\right)=0 \quad \text { for all } N \subset G_{\lambda} \text { with } \mathscr{L}^{n}(N)=0 ;
\end{gathered}
$$

and moreover, for every bounded $\psi \in \mathrm{C}^{1}\left((\operatorname{Span} \pi)^{\perp}\right)$ with $\operatorname{Lip}(\psi) \leq 1$, there holds

$$
\left|T_{y}(\psi)-T_{x}(\psi)\right| \leq \lambda|y-x|, \quad \text { whenever } x, y \in G_{\lambda} \text { are Lebesgue points of } \Phi_{\psi}
$$

and $T_{x}(\psi)=\Phi_{\psi}(x), T_{y}(\psi)=\Phi_{\psi}(y)$ are understood as the corresponding Lebesgue values.

Proof. We only treat the case $r=1$, as the general case follows by a simple scaling argument, and we first observe that $G_{\lambda}$ is closed in $\mathbf{B}_{1 / 2}^{\pi}$. By (4.2) the measure $\mu:=\mathrm{p}_{\sharp}\|T\|-m \mathscr{L}^{n}$ is non-negative on $\mathbf{B}_{1}^{\pi}$, and the non-centered maximal function of $\mu$ is given by

$$
\mathbf{m} \mu(x):=\sup _{\widetilde{x}, \varrho} \frac{\mu\left(\widetilde{x}+\mathbf{B}_{\varrho}^{\pi}\right)}{\varrho^{n}}=\sup _{\widetilde{x}, \varrho} \mathbf{E}(T, \widetilde{x}, \varrho, \pi) \quad \text { for } x \in \mathbf{B}_{1 / 2}^{\pi},
$$

where here and in the following the suprema in $\widetilde{x}, \varrho$ are taken over all $x \in \mathbf{B}_{1 / 2}^{\pi}$ and $\varrho \in(0,1 / 2)$ such that the ball $\widetilde{x}+\mathbf{B}_{\varrho}^{\pi}$ contains $x$. In view of $G_{\lambda}=\left\{x \in \mathbf{B}_{1 / 2}^{\pi}\right.$ : $\left.\mathbf{m} \mu(x) \leq \gamma \lambda^{2}\right\}$, the estimates (5.13) and (5.14) then follow from well-known maximalfunction arguments (compare [19, Section 6.6.2]).

For $\psi$ as in the last statement we now work with the BV-function $\Phi_{\psi}$ from Lemma 5.4. Then, by (2.18) in combination with (4.2) and the definition of $G_{\lambda}$ we get

$$
\sup _{\widetilde{x}, \varrho} \frac{\|T\|\left(\widetilde{x}+\mathbf{C}_{\varrho}^{\pi}\right)}{\varrho^{n}} \leq m \omega_{n}+\sup _{\widetilde{x}, \varrho} \mathbf{E}(T, \widetilde{x}, \varrho, \pi) \leq m \omega_{n}+\gamma \leq C \quad \text { for } x \in G_{\lambda},
$$

and, involving the BV estimate (5.11) in the second step, we can conclude

$$
\mathbf{m}\left|\mathrm{D} \Phi_{\psi}\right|(x)=\sup _{\widetilde{x}, \varrho} \frac{\left|\mathrm{D} \Phi_{\psi}\right|\left(\widetilde{x}+\mathbf{B}_{\varrho}^{\pi}\right)}{\varrho^{n}} \leq C \sup _{\widetilde{x}, \varrho}\left[\frac{\mu\left(\widetilde{x}+\mathbf{C}_{\varrho}^{\pi}\right)}{\varrho^{n}}\right]^{1 / 2} \leq C \gamma^{1 / 2} \lambda \quad \text { for } x \in G_{\lambda} .
$$


For Lebesgue points $x, y \in G_{\lambda}$ of $\Phi_{\psi}$ we now make use of another well-known property of maximal functions (compare again [19, Section 6.6.2]) to arrive at

$$
\left|\Phi_{\psi}(y)-\Phi_{\psi}(x)\right| \leq C\left[\mathbf{m}\left|\mathrm{D} \Phi_{\psi}\right|(x)+\mathbf{m}\left|\mathrm{D} \Phi_{\psi}\right|(y)\right]|y-x| \leq C \gamma^{1 / 2} \lambda|y-x| .
$$

Hence, choosing $\gamma \leq \min \left\{C^{-2}, \omega_{n}\right\}$ (with the constant $C$ appearing in the last line) and recalling the definition of $\Phi_{\psi}$, we have established (5.15) and the lemma.

We now observe that, if also the lower density bound (4.3) is in force, then at $\mathscr{L}^{n}$-a. e. $x \in \mathbf{B}_{1}^{\pi}$, the 0 -dimensional slices $T_{x}$ are given by $\sum_{i=1}^{N} \alpha_{i} \delta_{y_{i}}$ for some $N \in \mathbb{N}$, $y_{i} \in(\operatorname{Span} \pi)^{\perp}$, and $\alpha_{i} \in \mathbb{Z}$ with $\left|\alpha_{i}\right| \geq m$ and $\sum_{i=1}^{N} \alpha_{i}=m$ (where of course $N, y_{i}$ and $\alpha_{i}$ depend all on $x$ ). This observation will now be used to construct a single-valued approximating Lipschitz graph and to give a

Proof of Lemma 5.3. We first use the fact that any projection decreases mass, (a consequence of) the slicing theorem [5, Theorem 5.6], and finally (2.18) in combination with (4.2) and the definition of $G_{\lambda}$ from Lemma 5.5. In this way, recalling also that we have chosen $\gamma \leq \omega_{n}$, we get

$$
\mathbf{M}\left(T_{x}\right) \leq \mathbf{M}(\langle T, \mathrm{p}, x\rangle) \leq \liminf _{\varrho \searrow 0} \frac{1}{\omega_{n} \varrho^{n}}\|T\|\left(x+\mathbf{C}_{\varrho}^{\pi}\right) \leq m+\frac{\gamma \lambda^{2}}{2 \omega_{n}} \leq m+\frac{1}{2}
$$

for $\mathscr{L}^{n}$-a. e. $x \in G_{\lambda}$. Using the observation preceding the proof, we conclude that, for $\mathscr{L}^{n}$-a. e. $x \in G_{\lambda}$ the slice $T_{x}$ collapses to a point mass, i. e. there exists some $f(x) \in$ $(\operatorname{Span} \pi)^{\perp}$ such that $T_{x}=m \delta_{f(x)}$. We now choose countable dense sets $\left\{a_{1}, a_{2}, \ldots\right\}$ and $\left\{\chi_{1}, \chi_{2}, \ldots\right\}$ in $\mathbf{B}_{1}^{\perp \pi}$ and $\left\{\chi \in \mathrm{C}^{1}(\mathbb{R}): \chi\right.$ is bounded with $\left.\operatorname{Lip}(\chi) \leq 1\right\}$, respectively (where in the latter case density is understood with respect to the sup-norm). Setting $\psi_{i, j}(z):=\chi_{j}\left(\left\langle a_{i}, z\right\rangle\right)$ we obtain bounded $\psi_{i, j} \in \mathrm{C}^{1}\left((\operatorname{Span} \pi)^{\perp}\right)$ with $\operatorname{Lip}\left(\psi_{i, j}\right) \leq 1$, and — having reduced to the countably many $\psi$ — we can find a Borel subset $\widetilde{G}_{\lambda}$ of $G_{\lambda}$ with full $\mathscr{L}^{n}$-measure such that the $x \in \widetilde{G}_{\lambda}$ are all Lebesgue points for all $\Phi_{\psi_{i, j}}$ with $T_{x}\left(\psi_{i, j}\right)=m \chi_{j}\left(\left\langle f(x), a_{i}\right\rangle\right)$. We record the equality

$$
m|f(y)-f(x)|=\sup _{i, j \in \mathbb{N}}\left|T_{y}\left(\psi_{i, j}\right)-T_{x}\left(\psi_{i, j}\right)\right| \quad \text { for } x, y \in \widetilde{G}_{\lambda},
$$

which indeed follows easily, when we use, for $f(x) \neq f(y)$, the $a_{i}$ and $\chi_{j}$ to approximate $a:=\frac{f(y)-f(x)}{|f(y)-f(x)|}$ and some 1-Lipschitz function $\chi$ with $\chi(\langle a, f(x)\rangle)=0$ and $\chi(\langle a, f(y)\rangle)=|f(y)-f(x)|$. Next we we combine (5.16) and (5.15) to get

$$
|f(y)-f(x)| \leq \lambda|y-x| \quad \forall x, y \in \widetilde{G}_{\lambda} .
$$

Possibly changing the values of $f$ on the Lebesgue null set $G_{\lambda} \backslash \widetilde{G}_{\lambda}$, we use the Kirszbraun extension theorem (see [35]) to get a $\lambda$-Lipschitz function $f$ on all of $\mathbf{B}_{r / 2}^{\pi}$ with $T_{x}=m \delta_{f(x)}$ for all $x \in \widetilde{G}_{\lambda}$. We infer that $m \llbracket$ Graph $f \rrbracket$ and $T$ coincide on $\mathrm{p}^{-1}\left(\widetilde{G}_{\lambda}\right)$, and then, taking (5.14) into account, we obtain (5.8). Via (5.13) we also 
get

$$
\left\|T-T^{\mathrm{g}}\right\|\left(\mathbf{C}_{1 / 2}^{\pi}\right) \leq C \mathscr{L}^{n}\left(\mathbf{B}_{1 / 2}^{\pi} \backslash G_{\lambda}\right)+\|T\|\left(\mathrm{p}^{-1}\left(\mathbf{B}_{1 / 2}^{\pi} \backslash G_{\lambda}\right)\right) \leq \frac{C}{\gamma \lambda^{2}} \mathbf{E}(T, 0,1, \pi),
$$

and hence we arrive at the claim (5.9). Finally, taking the bound (5.1) from the vertical separation lemma and a radial truncation argument into account, we can assume that the oscillation bound in (5.10) holds.

Remark 5.6. Clearly, the bound $\operatorname{Lip}(f) \leq \lambda$ in Lemma 5.3 implies the bound for $\mathrm{D} f$ in the operator norm

$$
|\mathrm{D} f(x) \xi| \leq \lambda|\xi| \quad \text { for all } \xi \in \operatorname{Span} \pi \text { and } \mathscr{L}^{n} \text {-a. e. } x \in \mathbf{B}_{r / 2}^{\pi} .
$$

For the Hilbert-Schmidt norm of $\mathrm{D} f$ in the sense of (2.2) we infer

$$
\underset{\mathbf{B}_{r / 2}^{\pi}}{\operatorname{ess} \sup }|\mathrm{D} f| \leq \sqrt{n} \lambda \text {. }
$$

\section{COMPARISON With haRMONIC FUNCTIONS AND EXCESS DECAY}

Our main estimates, which will eventually imply regularity, are contained in the following proposition.

Proposition 6.1 (excess improvement). There exists a positive constant $C_{*}$ depending only on $n$ and $m$, and further for every $\tau \in\left(0, \frac{1}{16}\right]$ a constant $\varepsilon_{1} \in(0,1]$, depending only on $n, m$ and $\tau$, with the following property: whenever we have $0 \in \operatorname{spt} T$ and Assumption 4.1 holds for $T$ on $\mathbf{C}_{r}^{\pi}$ up to $\varepsilon \leq \varepsilon_{1}$, then there exists an n-plane $\pi^{(1)}$ in $\mathcal{H}$ with

$$
\left|\pi^{(1)}-\pi\right| \leq C_{*} \mathbf{E}(T, 0, r, \pi)^{3 /(40 n)} \leq \frac{1}{2}
$$

such that, for $T_{r / 2}:=T\left\llcorner\mathbf{C}_{r / 2}^{\pi}\right.$, we have

$$
\mathbf{E}\left(T_{r / 2}, 0, \tau r, \pi^{(1)}\right) \leq C_{*} \tau^{2} \mathbf{E}(T, 0, r, \pi)
$$

and $d^{7}$

$$
\mathbf{C}_{\tau r}^{\varpi} \cap \operatorname{spt} T_{r / 2} \subset \mathbf{C}_{2 \tau r}^{\pi} \quad \text { whenever }|\varpi-\pi| \leq C_{*} \mathbf{E}(T, 0, r, \pi)^{1 /(20 n)} .
$$

The following proof of the proposition follows once more the arguments of [32]. However, we will also involve some technical adaptions of the arguments, which are inspired by $[8,31]$; in particular, we will avoid the mollification procedure performed in [32], and we will show that global $\mathrm{W}^{1, p}$-estimates can substitute the global $\mathrm{C}^{1, \alpha}$ estimates used in [32].

Proof of Proposition 6.1. We fix $\tau \in\left(0, \frac{1}{16}\right]$ and work under the assumptions of the proposition, where the number $\varepsilon_{1} \in(0,1]$ with $\varepsilon_{1} \leq \varepsilon_{0}$ will only be fixed at the very end of our reasoning. Throughout this proof we will always abbreviate

$$
\mathbf{E}:=\mathbf{E}(T, 0, r, \pi)
$$

\footnotetext{
${ }^{7}$ The specific bound for $|\varpi-\pi|$ will be used conveniently in Proposition 6.3 .
} 
and we record that by (4.4) we have $\mathbf{E} \leq \varepsilon_{1} \leq 1$. Moreover, we can and do assume $\mathbf{E}>0$, as otherwise $\vec{T} \equiv \pi$ holds $\|T\|$-a. e. on $\mathbf{C}_{r}^{\pi}$ and the claims are trivially true for $\pi^{(1)}=\pi$.

Step 1. (Lipschitz approximation) We denote by $f: \mathbf{B}_{r / 2}^{\pi} \rightarrow(\operatorname{Span} \pi)^{\perp}$ the Lipschitz function of Lemma 5.3 corresponding to the choices

$$
\delta:=\frac{1}{20 n} \quad \text { and } \quad \lambda:=\mathbf{E}^{\delta} \leq 1 .
$$

We will widely work with the currents

$$
T^{\mathrm{g}}=m \llbracket \text { Graph } f \rrbracket \quad \text { and } \quad S:=m \llbracket \text { Graph } h \rrbracket,
$$

where the harmonic function $h: \mathbf{B}_{\sigma}^{\pi} \rightarrow(\operatorname{Span} \pi)^{\perp}$ and the radius $\sigma \in(r / 4, r / 2]$ will only eventually be constructed. We will make frequent use of the following two conventions: We will understand that the orienting $n$-vectors $\vec{T}^{\mathrm{g}}$ and $\vec{S}$, respectively, are extended to all of $\mathbf{C}_{r / 2}^{\pi}$ and $\mathbf{C}_{\sigma}^{\pi}$, constant in the directions from (Span $\left.\pi\right)^{\perp}$ (so, we can also consider them as functions of a variable which runs in $\mathbf{B}_{r / 2}^{\pi}$ or $\left.\mathbf{B}_{\sigma}^{\pi}\right)$. Moreover, when $R$ is any of the currents $T, T^{\mathrm{g}}, S$, we will often abbreviate $R_{\eta}:=R\left\llcorner\mathbf{C}_{\eta}^{\pi}\right.$ and $\partial R_{\eta}:=\partial\left(R\left\llcorner\mathbf{C}_{\eta}^{\pi}\right)\right.$.

Now we first observe that with the preceding choices (5.9) reads

$$
\left\|T^{\mathrm{g}}-T\right\|\left(\mathbf{C}_{r / 2}^{\pi}\right) \leq C r^{n} \mathbf{E}^{1-2 \delta} .
$$

By (2.32), (5.17), the area formula of (2.4), and the representation formula (2.13) we have

$$
\int_{\mathbf{B}_{r / 2}^{\pi}}|\mathrm{D} f|^{2} \mathrm{~d} \mathscr{L}^{n} \leq C \int_{\mathbf{B}_{r / 2}^{\pi}}\left|\vec{T}^{\mathrm{g}}-\pi\right|^{2} \mathrm{~J}_{n}(\mathrm{D} F) \mathrm{d} \mathscr{L}^{n}=\frac{C}{m}\left(\frac{r}{2}\right)^{n} \mathbf{E}\left(T^{\mathrm{g}}, 0, r / 2, \pi\right) .
$$

Relying on the alternative form (2.18) of the excess, on (6.6), and on $\left(\mathrm{p}^{\pi}\right)_{\sharp} T_{r / 2}=$ $\left(\mathrm{p}^{\pi}\right)_{\sharp} T_{r / 2}^{\mathrm{g}}$ we moreover find

$$
\left(\frac{r}{2}\right)^{n} \mathbf{E}\left(T^{\mathrm{g}}, 0, r / 2, \pi\right) \leq\left(\frac{r}{2}\right)^{n} \mathbf{E}(T, 0, r / 2, \pi)+C r^{n} \mathbf{E}^{1-2 \delta} \leq C r^{n} \mathbf{E}^{1-2 \delta},
$$

where the last inequality is a consequence of (4.2). Combining the last two estimates we arrive at

$$
\int_{\mathbf{B}_{r / 2}^{\pi}}|\mathrm{D} f|^{2} \mathrm{~d} \mathscr{L}^{n} \leq C r^{n} \mathbf{E}^{1-2 \delta} .
$$

Step 2. (Harmonic approximation of $f$ ) Identifying balls in $\mathbb{R}^{n}$ with balls in Span $\pi$, let $h \in \mathrm{C}\left(\overline{\mathbf{B}_{\sigma}^{\pi}},(\operatorname{Span} \pi)^{\perp}\right)$ be the harmonic function coinciding with $f$ on $\partial \mathbf{B}_{\sigma}^{\pi}$ given by Theorem 3.3. Thanks to (3.10) and (3.11) it satisfies

$$
\sup _{\mathbf{B}_{\eta}^{\pi}}|\mathrm{D} h|^{2} \leq \frac{C}{(\sigma-\eta)^{n}} \int_{\mathbf{B}_{\sigma}^{\pi}}|\mathrm{D} h|^{2} \mathrm{~d} \mathscr{L}^{n} \quad \text { for } 0<\eta<\sigma,
$$




$$
\sup _{\mathbf{B}_{2 \tau r}^{\pi}}|\mathrm{D} h-\mathrm{D} h(0)|^{2} \leq C \frac{\tau^{2}}{r^{n}} \int_{\mathbf{B}_{\sigma}^{\pi}}|\mathrm{D} h|^{2} \mathrm{~d} \mathscr{L}^{n} .
$$

In addition, (3.9) in combination with (6.7) gives

$$
\int_{\mathbf{B}_{\sigma}^{\pi}}|\mathrm{D} h|^{2} \mathrm{~d} \mathscr{L}^{n} \leq C r^{n} \mathbf{E}^{1-2 \delta} .
$$

Since $\sigma \leq r$, we can use (3.12) in combination with (5.17) and (6.4) to find

$$
\int_{\mathbf{B}_{\sigma}^{\pi}}|\mathrm{D} h|^{p} \mathrm{~d} \mathscr{L}^{n} \leq C \sigma^{n} \sup _{\mathbf{B}_{\sigma}^{\pi}}|\mathrm{D} f|^{p} \leq C r^{n} \mathbf{E}^{\delta p}
$$

for $1<p<\infty$, where $C$ depends only on $n$ and $p$.

Step 3. (Admissibility of the graph currents) At this stage let us affirm that the definitions in (6.5) do indeed define integral currents $T^{\mathrm{g}}$ and $S$ in $\mathbf{C}_{r / 2}^{\pi}$ and $\mathbf{C}_{\sigma}^{\pi}$, respectively. Indeed, as $f$ is globally Lipschitz, Graph $f$ has finite $\mathscr{H}^{n}$-measure, and $T^{\mathrm{g}}=\llbracket$ Graph $f \rrbracket$ is a well-defined integer-rectifiable current with finite mass; compare Section 2.4. Moreover, as pushforward commutes with the boundary operator, we have the equality

$$
\partial T_{\eta}^{\mathrm{g}}=\partial\left(m F_{\sharp} \llbracket \mathbf{B}_{\eta}^{\pi} \rrbracket\right)=m F_{\sharp} \partial \llbracket \mathbf{B}_{\eta}^{\pi} \rrbracket \quad \text { for } 0<\eta \leq r / 2,
$$

from which we read off that also $\partial T_{\eta}^{\mathrm{g}}$ and in particular $\partial T^{\mathrm{g}}=\partial T_{r / 2}^{\mathrm{g}}$ has finite mass. This shows $T^{\mathrm{g}} \in \mathbf{I}_{n}(\mathcal{H})$, but the same reasoning does not straightforwardly work for $S$; for $h$ is only locally, but not globally Lipschitz on $\mathbf{B}_{\sigma}^{\pi}$. We will overcome this point in the following by a technical extra argument, and as a side benefit we will establish the equality

$$
\partial S_{\sigma}=m F_{\sharp} \partial \llbracket \mathbf{B}_{\sigma}^{\pi} \rrbracket,
$$

which follows in essence from the coincidence of $h$ and $f$ on the boundary of $\mathbf{B}_{\sigma}^{\pi}$.

Indeed, the area formula (2.4), the estimate $\mathrm{J}_{n}(\mathrm{D} H) \leq|\mathrm{D} H|^{n} \leq C\left(1+|\mathrm{D} h|^{n}\right)$, and the $\mathrm{L}^{n}$-integrability of $\mathrm{D} h$ from (6.11) imply $\mathscr{H}^{n}(\mathrm{Graph} h)<\infty$, and thus $S=S_{\sigma}=m \llbracket$ Graph $h \rrbracket$ is well-defined and integer-rectifiable with finite mass. Next, for every given $\kappa>0$ we consider a smooth cut-off function $\varphi_{\kappa}$ on $\mathbf{B}_{\sigma}^{\pi}$, which is identically 1 on $\mathbf{B}_{\sigma-\kappa}^{\pi}$, identically 0 in a neighborhood of $\partial \mathbf{B}_{\sigma}^{\pi}$ and with $\left|\mathrm{D} \varphi_{\kappa}\right| \leq 2 \kappa^{-1}$. Considering $h_{\kappa}:=\varphi_{\kappa} h+\left(1-\varphi_{\kappa}\right) f$ as a global Lipschitz map on $\mathbf{B}_{\sigma}^{\pi}$, the current $W_{\kappa}=m \llbracket \operatorname{Graph} h_{\kappa} \rrbracket \in \mathrm{I}_{n}(\mathcal{H})$ is well-defined with $\partial W_{\kappa}=m F_{\sharp} \partial \llbracket \mathbf{B}_{\sigma}^{\pi} \rrbracket$. In order to justify (6.13), it now suffices to show $\lim _{\kappa} \backslash 0\left\|S-W_{\kappa}\right\|\left(\mathbf{C}_{\sigma}^{\pi}\right)=0$, since then $\partial S=$ $\lim _{\kappa \searrow 0} \partial W_{\kappa}=m F_{\sharp} \partial \llbracket \mathbf{B}_{\sigma}^{\pi} \rrbracket$ follows. We now record $\left|\mathrm{D} h_{\kappa}\right| \leq|\mathrm{D} h|+|\mathrm{D} f|+2 \kappa^{-1}|h-f|$ and observe that $W_{\kappa}$ coincides with $S$ on $\mathbf{C}_{\sigma-\kappa}^{\pi}$. Thus, by the area formula (2.4) we conclude

$$
\left\|S-W_{\kappa}\right\|\left(\mathbf{C}_{\sigma}^{\pi}\right) \leq C \underbrace{\int_{\mathbf{B}_{\sigma}^{\pi} \backslash \mathbf{B}_{\sigma-\kappa}^{\pi}}\left(1+|\mathrm{D} h|^{n}+|\mathrm{D} f|^{n}\right) \mathrm{d} \mathscr{L}^{n}}_{I_{1}}+C \underbrace{\kappa^{-n} \int_{\mathbf{B}_{\sigma}^{\pi} \backslash \mathbf{B}_{\sigma-\kappa}^{\pi}}|h-f|^{n} \mathrm{~d} \mathscr{L}^{n}}_{I_{2}} .
$$


In order to estimate $I_{2}$ we use polar coordinates:

$$
I_{2} \leq C \kappa^{-n} \int_{\partial \mathbf{B}_{1}^{\pi}} \kappa\left(\max _{s \in[\sigma-\kappa, \sigma]}|h(s x)-f(s x)|^{n}\right) \mathrm{d} \mathscr{H}^{n-1}(x) .
$$

However, the function $s \mapsto h(s x)-f(s x)$ vanishes for $s=\kappa$, thus via 1-dimensional integration and Hölder's inequality we achieve

$$
\max _{s \in[\sigma-\kappa, \sigma]}|h(s x)-f(s x)|^{n} \leq \kappa^{n-1} \int_{\sigma-\kappa}^{\sigma}|\mathrm{D} h(s x)-\mathrm{D} f(s x)|^{n} \mathrm{~d} s .
$$

We therefore conclude

$$
I_{2} \leq C \int_{\mathbf{B}_{\sigma}^{\pi} \backslash \mathbf{B}_{\sigma-\kappa}^{\pi}}|\mathrm{D} f-\mathrm{D} h|^{n} \mathrm{~d} \mathscr{L}^{n} \leq C I_{1} .
$$

Clearly, $I_{1}$ converges to 0 when we send $\kappa$ to 0 , hence we can conclude the convergence $\lim _{\kappa \searrow 0}\left\|S-W_{\kappa}\right\|\left(\mathbf{C}_{\sigma}^{\pi}\right)=0$, and the claim (6.13) follows.

Step 4. (Construction of a comparison current) In spite of (6.13) we will need to modify the boundary of $S$ in order to properly use it as a comparison current for the minimality of $T$. This is now achieved by the following choice of a good radius $\sigma$ and the homotopy construction of Lemma A.3. We set

$$
Q:=\min \left\{q \in \mathbb{N}: q \geq \mathbf{E}^{-3 \delta}\right\}
$$

and $r(i):=\frac{r}{4}+i \frac{r}{4 Q}$ for $i \in\{0,1,2, \ldots, Q\}$. Clearly, we then have $\mathbf{E}^{-3 \delta} \leq Q \leq 2 \mathbf{E}^{-3 \delta}$ and $r / 4=r(0) \leq r(1) \leq r(2) \leq \ldots \leq r(Q-1) \leq r(Q)=r / 2$, and we can find some $i_{0} \in\{0,1,2, \ldots, Q-1\}$ with

$$
\left\|T^{\mathrm{g}}-T\right\|\left(\mathbf{C}_{r\left(i_{0}+1\right)}^{\pi} \backslash \mathbf{C}_{r\left(i_{0}\right)}^{\pi}\right) \leq \frac{1}{Q}\left\|T^{\mathrm{g}}-T\right\|\left(\mathbf{C}_{r / 2}^{\pi} \backslash \mathbf{C}_{r / 4}^{\pi}\right) .
$$

Furthermore, slicing as in $(2.8)^{8}$ we deduce that $\partial T_{\eta}$ has finite mass for $\mathscr{L}^{1}$-a. e. $\eta \in(0, r / 2)$ and that we have

$$
\int_{0}^{r / 2}\left\|\partial T_{\eta}\right\|(\mathcal{H} \backslash \operatorname{Graph} f) \mathrm{d} \mathscr{L}^{1}(\eta) \leq\|T\|\left(\mathbf{C}_{r / 2}^{\pi} \backslash \text { Graph } f\right) .
$$

In particular, we can fix a radius $\sigma$ with $\mathbf{M}\left(\partial T_{\sigma}\right)<\infty$,

$$
\frac{r\left(i_{0}\right)+r\left(i_{0}+1\right)}{2} \leq \sigma \leq r\left(i_{0}+1\right)
$$

and

$$
\left\|\partial T_{\sigma}\right\|(\mathcal{H} \backslash \text { Graph } f) \leq \frac{2}{r\left(i_{0}+1\right)-r\left(i_{0}\right)}\|T\|\left(\mathbf{C}_{r / 2}^{\pi} \backslash \text { Graph } f\right)
$$

\footnotetext{
${ }^{8}$ Indeed, relying also on (4.1) we here use (2.8) with the slicing map $\psi=-\left|\mathrm{p}^{\pi}\right|$ and consequently with the slices $\partial\left(T\llcorner\{\psi>-\eta\})=\partial T_{\eta}\right.$.
} 
Taking into account $\left(r\left(i_{0}+1\right)-r\left(i_{0}\right)\right) / 2=r /(8 Q) \geq r \mathbf{E}^{3 \delta} / 16$ the last inequality implies

$$
\left\|\partial T_{\sigma}\right\|(\mathcal{H} \backslash \operatorname{Graph} f) \leq \frac{16 \mathbf{E}^{-3 \delta}}{r}\|T\|\left(\operatorname{spt} T_{r / 2} \backslash \text { Graph } f\right) .
$$

Now on the one hand (4.2) gives

$$
\|T\|\left(\operatorname{spt} T_{r / 2}\right) \geq\left\|\left(p^{\pi}\right)_{\sharp} T\right\|\left(\mathbf{B}_{r / 2}^{\pi}\right)=m \omega_{n}(r / 2)^{n},
$$

and on the other hand we have from (6.5) and (6.6)

$$
\|T\|\left(\operatorname{spt} T_{r / 2} \backslash \text { Graph } f\right) \leq \| T-m \llbracket \text { Graph } f \rrbracket \|\left(\mathbf{C}_{r / 2}^{\pi}\right) \leq C_{1} \mathbf{E}^{1-2 \delta} r^{n},
$$

where the constant $C_{1}$ depends only on $n$ and $m$. Assuming the smallness condition

$$
C_{1} \mathbf{E}^{1-2 \delta}<m \omega_{n} 2^{-n}
$$

(this requirement and similar ones will be justified at the end of the proof) we thus find that the intersection $\left(\operatorname{spt} T_{r / 2}\right) \cap$ Graph $f$ contains at least one point $z_{0}$. In particular, we have $\mathrm{q}^{\pi}\left(z_{0}\right)=f\left(\mathrm{p}^{\pi}\left(z_{0}\right)\right)$, and from (5.1) and (5.10) we get

$$
\sup _{\operatorname{spt} \partial T_{\sigma}}\left|\mathrm{q}^{\pi}-f \circ \mathrm{p}^{\pi}\right| \leq \sup _{\operatorname{spt} T_{r / 2}}\left|\mathrm{q}^{\pi}-\mathrm{q}^{\pi}\left(z_{0}\right)\right|+\sup _{\mathbf{B}_{r / 2}}\left|f-f\left(\mathrm{p}^{\pi}\left(z_{0}\right)\right)\right| \leq \operatorname{Cr} \mathbf{E}^{1 /(2 n)}
$$

Consequently, $\partial T_{\sigma}$ satisfies (A.4) (with $K$ equal to the right hand side of the last estimate), and we can apply Lemma A.3 (with $\partial T_{\sigma}$ in place of $T$ and the corresponding restriction of $f$ ) to obtain an $n$-dimensional current $V \in \mathrm{I}_{n}(\mathcal{H})$ with

$$
\operatorname{spt} V \subset\left(\mathrm{p}^{\pi}\right)^{-1}\left(\partial \mathbf{B}_{\sigma}^{\pi}\right)
$$

such that we have

$$
\partial V=\partial T_{\sigma}-F_{\sharp}\left(\mathrm{p}^{\pi}\right)_{\sharp} \partial T_{\sigma}
$$

and

$$
\mathbf{M}(V) \leq C r \mathbf{E}^{1 /(2 n)}(1+\operatorname{Lip}(f))^{n-1}\left\|\partial T_{\sigma}\right\|(\mathcal{H} \backslash \text { Graph } f) .
$$

By (4.2) we have $\left(\mathrm{p}^{\pi}\right)_{\sharp} \partial T_{\sigma}=\partial\left(\mathrm{p}^{\pi}\right)_{\sharp} T_{\sigma}=m \partial \llbracket \mathbf{B}_{\sigma}^{\pi} \rrbracket$, and in view of $(6.12)$, (6.13) the above formula for $\partial V$ yields

$$
\partial V=\partial T_{\sigma}-\partial T_{\sigma}^{\mathrm{g}}=\partial T_{\sigma}-\partial S_{\sigma} .
$$

Moreover, taking into account $\operatorname{Lip}(f) \leq 1,(6.15)$, and (6.16) the estimate for $\mathbf{M}(V)$ simplifies to

$$
\mathbf{M}(V) \leq C r^{n} \mathbf{E}^{1+1 /(2 n)-5 \delta}=C r^{n} \mathbf{E}^{1+5 \delta} .
$$

Step 5. (Excess estimates) At this stage we ultimately start the main line of our estimates. Abbreviating

$$
\mathrm{X}:=\int_{\mathbf{C}_{\sigma}^{\pi}}|\vec{T}-\vec{S}|^{2} \mathrm{~d}\|T\| \quad \text { and } \quad \mathrm{Y}:=\int_{\mathbf{C}_{2 \tau r}^{\pi}}|\vec{S}-\vec{S}(0)|^{2} \mathrm{~d}\|T\|
$$


we clearly have (because $2 \tau r \leq r / 4 \leq \sigma$ )

$$
\int_{\mathbf{C}_{2 \tau r}^{\pi}}|\vec{T}-\vec{S}(0)|^{2} \mathrm{~d}\|T\| \leq 2 \mathrm{X}+2 \mathrm{Y} .
$$

To control X we use that the orientations $\vec{T}$ and $\vec{S}$ satisfy $|\vec{T}|=|\vec{S}|=1$ and consequently $\frac{1}{2}|\vec{T}-\vec{S}|^{2}+\langle\vec{S}, \vec{T}\rangle=1$. All in all we thus find

$$
\begin{aligned}
\mathbf{M}\left(T_{\sigma}\right)-\mathbf{M}\left(S_{\sigma}\right) & =\int_{\mathbf{C}_{\sigma}^{\pi}}\left[\frac{1}{2}|\vec{T}-\vec{S}|^{2}+\langle\vec{S}, \vec{T}\rangle\right] \mathrm{d}\|T\|-\int_{\mathbf{C}_{\sigma}^{\pi}}\langle\vec{S}, \vec{S}\rangle \mathrm{d}\|S\| \\
& =\frac{1}{2} \int_{\mathbf{C}_{\sigma}^{\pi}}|\vec{T}-\vec{S}|^{2} \mathrm{~d}\|T\|-\left(S_{\sigma}-T_{\sigma}\right)(\vec{S}) .
\end{aligned}
$$

Then, using (6.18) and (6.19) we can compare $T$ with $T+S_{\sigma}-T_{\sigma}+V$ to find

$$
\mathbf{M}\left(T_{\sigma}\right)-\mathbf{M}\left(S_{\sigma}\right) \leq \mathbf{M}(V) .
$$

Now, using in turn the preceding two estimates, (2.16) for the current $T_{\sigma}^{\mathrm{g}}-T_{\sigma}+V$ (which by (6.19) has zero boundary), and (6.20) we arrive at

$$
\begin{aligned}
\frac{1}{2} \mathrm{X} & \leq \mathbf{M}(V)+\left(S_{\sigma}-T_{\sigma}\right)(\vec{S}) \\
& =\mathbf{M}(V)-\left(T_{\sigma}^{\mathrm{g}}-T_{\sigma}+V\right)(\pi)+\left(S_{\sigma}-T_{\sigma}\right)(\vec{S}) \\
& \leq 2 \mathbf{M}(V)-\left(T_{\sigma}^{\mathrm{g}}-T_{\sigma}\right)(\pi)+\left(S_{\sigma}-T_{\sigma}\right)(\vec{S}) \\
& =2 \mathbf{M}(V)+\left(T_{\sigma}^{\mathrm{g}}-T_{\sigma}\right)(\vec{S}-\pi)+\left(S_{\sigma}-T_{\sigma}^{\mathrm{g}}\right)(\vec{S}) \\
& \leq C r^{n} \mathbf{E}^{1+5 \delta}+\mathrm{X}_{1}+\mathrm{X}_{2}
\end{aligned}
$$

where we have set

$$
\mathrm{X}_{1}:=\left(T_{\sigma}^{\mathrm{g}}-T_{\sigma}\right)(\vec{S}-\pi) \quad \text { and } \quad \mathrm{X}_{2}:=\left(S_{\sigma}-T_{\sigma}^{\mathrm{g}}\right)(\vec{S}) .
$$

Since $\sigma \leq r\left(i_{0}+1\right)$, from $(6.15)$ we have

$$
\left\|T^{\mathrm{g}}-T\right\|\left(\mathbf{C}_{\sigma}^{\pi} \backslash \mathbf{C}_{r\left(i_{0}\right)}^{\pi}\right) \leq \frac{1}{Q}\left\|T^{\mathrm{g}}-T\right\|\left(\mathbf{C}_{r / 2}^{\pi}\right),
$$

and by the choice of $Q$ and (6.6) we deduce

$$
\left\|T^{\mathrm{g}}-T\right\|\left(\mathbf{C}_{\sigma}^{\pi} \backslash \mathbf{C}_{r\left(i_{0}\right)}^{\pi}\right) \leq C r^{n} \mathbf{E}^{1+\delta} .
$$

We now involve (2.30), the elliptic estimate (6.8) (with $\eta=r\left(i_{0}\right)$ ), (6.10), and $\sigma-$ $r\left(i_{0}\right) \geq r /(8 Q) \geq \mathbf{E}^{3 \delta} /(16 r)$, where the last inequalities result from the above choices of $r\left(i_{0}\right)$ and $Q$. In this way we deduce

$$
\begin{aligned}
\sup _{\mathbf{C}_{r\left(i_{0}\right)}^{\pi}}|\vec{S}-\pi|^{2} \leq C \sup _{\mathbf{B}_{r\left(i_{0}\right)}^{\pi}}|\mathrm{D} h|^{2} & \leq \frac{C}{\left(\sigma-r\left(i_{0}\right)\right)^{n}} \int_{\mathbf{B}_{\sigma}^{\pi}}|\mathrm{D} h|^{2} \mathrm{~d} \mathscr{L}^{n} \\
& \leq C \mathbf{E}^{1-2 \delta-3 n \delta} \leq C_{2} \mathbf{E}^{3 \delta}
\end{aligned}
$$


with a constant $C_{2}$, which depends only on $n$ and $m$. Here, in the last step we exploited that $1-2 \delta-3 n \delta \geq 3 \delta$, which in turn is easily verified for the choice of $\delta$ from (6.4). From (6.23), (6.24) and (6.6) we get

$$
\left|\mathrm{X}_{1}\right| \leq 2\left\|T^{\mathrm{g}}-T\right\|\left(\mathbf{C}_{\sigma}^{\pi} \backslash \mathbf{C}_{r\left(i_{0}\right)}^{\pi}\right)+\left\|T^{\mathrm{g}}-T\right\|\left(\mathbf{C}_{r\left(i_{0}\right)}^{\pi}\right) \sup _{\mathbf{C}_{r\left(i_{0}\right)}^{\pi}}|\vec{S}-\pi| \leq C r^{n} \mathbf{E}^{1+\delta} .
$$

Keeping in mind that $T^{\mathrm{g}}=m \llbracket$ Graph $f \rrbracket$ and $S:=m \llbracket$ Graph $h \rrbracket$, we now rewrite $\mathrm{X}_{2}$ with the help of the area formula (2.4), (2.12) and (2.15). Then we compute and estimate the integrand in an orthonormal basis $\pi_{1}, \pi_{2}, \ldots, \pi_{n}$ of Span $\pi$, using the computations of Section 2.5 (see in particular (2.24) and (2.26)). In this way we obtain

$$
\begin{aligned}
\mathrm{X}_{2} & =\left(S_{\sigma}-T_{\sigma}^{\mathrm{g}}\right)(\vec{S})=m \mathscr{H}^{n}(\operatorname{Graph} h)-m \int_{\mathbf{C}_{\sigma}^{\pi} \cap \operatorname{Graph} f}\left\langle\vec{S}, \vec{T}^{\mathrm{g}}\right\rangle \mathrm{d} \mathscr{H}^{n} \\
& =m \int_{\mathbf{B}_{\sigma}^{\pi}}\left[\mathrm{J}_{n}(\mathrm{D} H)^{2}-\left\langle\bigwedge_{i=1}^{n}(\mathrm{D} H) \pi_{i}, \bigwedge_{i=1}^{n}(\mathrm{D} F) \pi_{i}\right\rangle\right] \frac{\mathrm{d} \mathscr{L}^{n}}{\mathrm{~J}_{n}(\mathrm{D} H)} \\
& =m \int_{\mathbf{B}_{\sigma}^{\pi}}\left[\langle\mathrm{D} h, \mathrm{D} h-\mathrm{D} f\rangle+\sum_{k=2}^{n}\left\langle[\mathrm{D} h]_{k}^{\pi},[\mathrm{D} h]_{k}^{\pi}-[\mathrm{D} f]_{k}^{\pi}\right\rangle\right] \frac{\mathrm{d} \mathscr{L}^{n}}{\mathrm{~J}_{n}(\mathrm{D} H)}
\end{aligned}
$$

For the first term in the last integrand (2.27) gives

$$
\frac{1}{\mathrm{~J}_{n}(\mathrm{D} H)}\langle\mathrm{D} h, \mathrm{D} h-\mathrm{D} f\rangle \leq\langle\mathrm{D} h, \mathrm{D} h-\mathrm{D} f\rangle+C \min \left\{|\mathrm{D} h|^{2}, 1\right\}|\mathrm{D} h||\mathrm{D} h-\mathrm{D} f| \text {. }
$$

For the second term we use (2.21), (2.26) (in form of the estimate $\left|[\mathrm{D} h]_{k}^{\pi}\right| / \mathrm{J}_{n}(\mathrm{D} H) \leq$ $\left.C \min \left\{|\mathrm{D} h|^{k}, 1\right\}\right)$, and the fact that $|\mathrm{D} f|$ is bounded via (5.17) and (6.4). In this way we establish

$$
\begin{aligned}
\frac{1}{\mathrm{~J}_{n}(\mathrm{D} H)} \sum_{k=2}^{n}\left\langle[\mathrm{D} h]_{k}^{\pi},[\mathrm{D} h]_{k}^{\pi}-[\mathrm{D} f]_{k}^{\pi}\right\rangle & \leq C \sum_{k=2}^{n} \min \left\{|\mathrm{D} h|^{k}, 1\right\}\left[|\mathrm{D} h|^{k}+|\mathrm{D} f|^{k}\right] \\
& \leq C \min \left\{|\mathrm{D} h|^{2}, 1\right\}\left[|\mathrm{D} h|^{2}+|\mathrm{D} h|^{n}+|\mathrm{D} f|^{2}\right] .
\end{aligned}
$$

When we indeed control the terms on the right hand side of (6.26) as just described and use Young's inequality, we arrive at

$$
\begin{aligned}
\mathrm{X}_{2} \leq m \int_{\mathbf{B}_{\sigma}^{\pi}}\langle\mathrm{D} h, \mathrm{D} h-\mathrm{D} f\rangle \mathrm{d} \mathscr{L}^{n} & \\
& +C \int_{\mathbf{B}_{\sigma}^{\pi}} \min \left\{|\mathrm{D} h|^{2}, 1\right\}\left[|\mathrm{D} f|^{2}+|\mathrm{D} h|^{2}+|\mathrm{D} h|^{n}\right] \mathrm{d} \mathscr{L}^{n} .
\end{aligned}
$$

Since $h$ is harmonic and $h-f$ vanish at $\partial \mathbf{B}_{\sigma}^{\pi}$, the first integral in (6.27) vanishes. For the other one we split the domain of integration, we exploit that $\sup _{\mathbf{B}_{\sigma}^{\pi}}|\mathrm{D} f| \leq$ 
$\sqrt{n} \mathbf{E}^{\delta} \leq \sqrt{n}$ holds by (5.17) and (6.4), and we find

$$
\begin{aligned}
\mathrm{X}_{2} \leq C\left[\left[\mathbf{E}^{2 \delta}+\mathbf{E}^{2 \delta^{2}}\right] \int_{\mathbf{B}_{\sigma}^{\pi} \cap\left\{|\mathrm{D} h| \leq \mathbf{E}^{\delta^{2}}\right\}}\right. & |\mathrm{D} h|^{2} \mathrm{~d} \mathscr{L}^{n} \\
& \left.+\int_{\mathbf{B}_{\sigma}^{\pi} \cap\left\{|\mathrm{D} h|>\mathbf{E}^{\left.\delta^{2}\right\}}\right.}\left[|\mathrm{D} h|^{2}+|\mathrm{D} h|^{n}\right] \mathrm{d} \mathscr{L}^{n}\right] .
\end{aligned}
$$

The next step is based on (2.32), on the fact that $\mathrm{J}_{n}(\mathrm{D} H)$ is bounded on $\{|\mathrm{D} h| \leq$ $1\}$, on the inequality $\left(\mathrm{p}^{\pi}\right)_{\sharp}\left[\|T\|\left\llcorner\mathbf{C}_{\sigma}^{\pi}\right] \geq\left\|\left(\mathrm{p}^{\pi}\right)_{\sharp} T\right\|\left\llcorner\mathbf{B}_{\sigma}^{\pi}=m \mathscr{L}^{n}\left\llcorner\mathbf{B}_{\sigma}^{\pi}\right.\right.\right.$, and on the definitions of $\mathrm{X}$ and $\mathbf{E}$. Indeed, these ingredients leave us with the estimate

$$
\begin{aligned}
\int_{\mathbf{B}_{\sigma}^{\pi} \cap\left\{|\mathrm{D} h| \leq \mathbf{E}^{\delta^{2}}\right\}}|\mathrm{D} h|^{2} \mathrm{~d} \mathscr{L}^{n} & \leq C \int_{\mathbf{B}_{\sigma}^{\pi}}|\vec{S}-\pi|^{2} \mathrm{~d} \mathscr{L}^{n} \\
& \leq C \int_{\mathbf{C}_{\sigma}^{\pi}}|\vec{S}-\pi|^{2} \mathrm{~d}\|T\| \\
& \leq C\left[\int_{\mathbf{C}_{\sigma}^{\pi}}|\vec{T}-\vec{S}|^{2} \mathrm{~d}\|T\|+\int_{\mathbf{C}_{r}^{\pi}}|\vec{T}-\pi|^{2} \mathrm{~d}\|T\|\right] \\
& \leq C\left[\mathrm{X}+r^{n} \mathbf{E}\right],
\end{aligned}
$$

where $C$ depends only on $n$ and $m$. Furthermore, when we notice $2+1 / \delta \geq n$ and use (6.11) with $p=2+1 / \delta$, we find

$$
\int_{\mathbf{B}_{\sigma}^{\pi} \cap\left\{|\mathrm{D} h|>\mathbf{E}^{\delta^{2}}\right\}}\left[|\mathrm{D} h|^{2}+|\mathrm{D} h|^{n}\right] \mathrm{d} \mathscr{L}^{n} \leq 2 \mathbf{E}^{-\delta} \int_{\mathbf{B}_{\sigma}^{\pi}}|\mathrm{D} h|^{2+1 / \delta} \mathrm{d} \mathscr{L}^{n} \leq C r^{n} \mathbf{E}^{1+\delta} .
$$

With the help of (6.29) and (6.30) we can control the right hand side of (6.28). Observing also $\mathbf{E}^{2 \delta} \leq \mathbf{E}^{\delta} \leq \mathbf{E}^{2 \delta^{2}}$ (since $\mathbf{E} \leq 1$ and $\delta \leq \frac{1}{2}$ ) we come out with

$$
\mathrm{X}_{2} \leq C \mathbf{E}^{2 \delta^{2}}\left[\mathrm{X}+r^{n} \mathbf{E}\right] .
$$

Collecting the estimates (6.22), (6.25), and (6.31) and observing also $\mathbf{E}^{5 \delta} \leq \mathbf{E}^{\delta} \leq \mathbf{E}^{2 \delta^{2}}$ we have in total

$$
\mathrm{X} \leq C_{3} \mathbf{E}^{2 \delta^{2}}\left[\mathrm{X}+r^{n} \mathbf{E}\right],
$$

where the constant $C_{3}$ depends only on $n$ and $m$. Imposing the smallness assumption

$$
C_{3} \mathbf{E}^{2 \delta^{2}} \leq \frac{1}{2}
$$

we can absorb one term, and as the final estimate for $X$, we can conclude even

$$
\mathrm{X} \leq 2 C_{3} r^{n} \mathbf{E}^{1+2 \delta^{2}} .
$$

Next we will derive an estimate for $Y$, which will be based on the following refined variant of (6.24). Indeed, we apply (2.31) and recall from Section 2.5 that $\ell \mapsto[\ell]_{1}^{\pi}$ is linear and $\left|[\ell]_{1}^{\pi}\right|=|\ell|$. Then we have

$$
\begin{aligned}
|\vec{S}-\vec{S}(0)| & \leq\left|\left(\pi+[\mathrm{D} h]_{1}^{\pi}\right)-\left(\pi+[\mathrm{D} h]_{1}^{\pi}(0)\right)\right|+C\left[|\mathrm{D} h(0)|^{2}+|\mathrm{D} h|^{2}\right] \\
& =|\mathrm{D} h-\mathrm{D} h(0)|+C\left[|\mathrm{D} h(0)|^{2}+|\mathrm{D} h|^{2}\right]
\end{aligned}
$$


on $\mathbf{B}_{\sigma}^{\pi}$. Taking into account also (6.9) and (6.8) (with $\eta=\sigma / 2$; notice $\sigma-\sigma / 2 \geq r / 8$ ) we see

$$
\begin{aligned}
\sup _{\mathbf{C}_{2 \tau r}^{\pi}}|\vec{S}-\vec{S}(0)|^{2} & \leq C\left[\sup _{\mathbf{B}_{2 \tau r}^{\pi}}|\mathrm{D} h-\mathrm{D} h(0)|^{2}+\sup _{\mathbf{B}_{\sigma / 2}^{\pi}}|\mathrm{D} h|^{4}\right] \\
& \leq C\left[\frac{\tau^{2}}{r^{n}} \int_{\mathbf{B}_{\sigma}^{\pi}}|\mathrm{D} h|^{2} \mathrm{~d} \mathscr{L}^{n}+\left(\frac{1}{r^{n}} \int_{\mathbf{B}_{\sigma}^{\pi}}|\mathrm{D} h|^{2} \mathrm{~d} \mathscr{L}^{n}\right)^{2}\right] .
\end{aligned}
$$

Recalling (6.29), (6.30), (6.33) the right hand side is controlled by

$$
\int_{\mathbf{B}_{\sigma}^{\pi}}|\mathrm{D} h|^{2} \mathrm{~d} \mathscr{L}^{n} \leq C r^{n} \mathbf{E}
$$

and hence we come out with

$$
\sup _{\mathbf{C}_{2 \tau r}^{\pi}}|\vec{S}-\vec{S}(0)|^{2} \leq C\left[\tau^{2} \mathbf{E}+\mathbf{E}^{2}\right]
$$

From (2.18) and (4.2) we now read off

$$
\|T\|\left(\mathbf{C}_{2 \tau r}^{\pi}\right)=r^{n}\left(\frac{1}{2} \mathbf{E}+m \omega_{n}(2 \tau)^{n}\right),
$$

and then we infer via (6.34), (6.35), and the inequalities $\tau \leq 1, \mathbf{E} \leq 1$

$$
\begin{aligned}
\mathrm{Y}=\int_{\mathbf{C}_{2 \tau r}^{\pi}}|\vec{S}-\vec{S}(0)|^{2} \mathrm{~d}\|T\| & \leq C r^{n}\left(\mathbf{E}+\tau^{n}\right)\left[\tau^{2} \mathbf{E}+\mathbf{E}^{2}\right] \\
& \leq C r^{n}\left[\tau^{n+2} \mathbf{E}+\mathbf{E}^{2}\right]
\end{aligned}
$$

Collecting (6.21), (6.33), and (6.36) we arrive at

$$
\int_{\mathbf{C}_{2 \tau r}^{\pi}}|\vec{T}-\vec{S}(0)|^{2} \mathrm{~d}\|T\| \leq C r^{n}\left[\tau^{n+2} \mathbf{E}+\mathbf{E}^{1+2 \delta^{2}}\right] .
$$

Step 6. (Tilting of planes and cylinders; conclusion) Next we turn to our claim (6.3), which follows via Lemma 5.2 from a suitable smallness assumption. Indeed, we consider an arbitrary $n$-plane $\varpi$ with

$$
|\varpi-\pi| \leq C_{*} \mathbf{E}^{1 /(20 n)}
$$

where $C_{*}$ will be fixed below. When we assume

$$
\mathbf{E} \leq \lambda_{0}^{-2 n} \quad \text { and } \quad C_{*} \mathbf{E}^{1 /(20 n)} \leq \frac{\tau}{12 n},
$$

Lemma 5.2 is applicable and immediately gives (6.3).

Turning to the claims (6.1) and (6.2) we now set

$$
\pi^{(1)}:=\vec{S}(0)
$$

As in the sequel we will fix $C_{*}$ larger than the quantity $\sqrt{C_{2}}$ from (6.24) above, the latter estimate gives the control

$$
\left|\pi^{(1)}-\pi\right| \leq C_{*} \mathbf{E}^{3 \delta / 2}=C_{*} \mathbf{E}^{3 /(40 n)} \leq C_{*} \mathbf{E}^{1 /(20 n)} .
$$


In addition, (6.39) implies $C_{*} \mathbf{E}^{1 /(20 n)} \leq \frac{1}{2}$, and thus (6.1) is established. Furthermore, (6.40) shows that the already proved claim (6.3) is applicable for $\varpi=\pi^{(1)}$. By (6.3) with this choice and by (6.37) we then have

$$
(\tau r)^{n} \mathbf{E}\left(T_{r / 2}, 0, \tau r, \pi^{(1)}\right) \leq \int_{\mathbf{C}_{2 \tau r}^{\pi}}|\vec{T}-\vec{S}(0)|^{2} \mathrm{~d}\|T\| \leq C r^{n}\left[\tau^{n+2}+\mathbf{E}^{2 \delta^{2}}\right] \mathbf{E} .
$$

Now we finally fix $C_{*}$ larger than $\sqrt{C_{2}}$ and twice the constant in the last estimate, which both depend only on $n$ and $m$. Then we obtain (6.2) by postulation of the smallness condition

$$
\mathbf{E}^{2 \delta^{2}} \leq \tau^{n+2}
$$

To conclude the proof of Proposition 6.1 we finally choose $\varepsilon_{1} \in\left(0, \varepsilon_{0}\right]$ small enough that the condition $\mathbf{E} \leq \varepsilon_{1}$ implies the previously assumed conditions (6.17), (6.32), (6.39), and (6.41). Recalling $\delta=\frac{1}{20 n}$ this choice can be achieved in such a way that $\varepsilon_{1}$ depends only on $n, m$, and $\tau$. The proof of Proposition 6.1 is now complete.

We next observe that in the situation of Proposition 6.1, Assumption 4.1 automatically carries over from scale $r$ to the smaller scale $\tau r$. More precisely, we have:

Lemma 6.2. Consider $\tau \in\left(0, \frac{1}{16}\right]$ and the corresponding number $\varepsilon_{1}$ from Proposition 6.1. If $0 \in \operatorname{spt} T$ and Assumption 4.1 holds for $T$ on $\mathbf{C}_{r}^{\pi}$ up to $\varepsilon \leq \varepsilon_{1}$, then it also holds with the same multiplicity $m$ for $T_{r / 2}:=T\left\llcorner\mathbf{C}_{r / 2}^{\pi}\right.$ on $\mathbf{C}_{\tau r}^{\pi^{(1)}}$ up to $\varepsilon$.

Proof. Since we require the same assumptions as for Proposition 6.1, the assertions of the proposition can be employed. By (6.1) the choice $\varpi=\pi^{(1)}$ in (6.3) is admissible and we have in particular $\mathbf{C}_{\tau r}^{\pi^{(1)}} \cap \operatorname{spt} T_{r / 2} \subset \mathbf{C}_{r / 2}^{\pi}$. This inclusion implies $\left(\partial T_{r / 2}\right)\left\llcorner\mathbf{C}_{\tau r}^{\pi^{(1)}}=(\partial T)\left\llcorner\left(\mathbf{C}_{r / 2}^{\pi} \cap \mathbf{C}_{\tau r}^{\pi^{(1)}}\right)\right.\right.$ and thus (4.1) carries over to $T_{r / 2}$ on $\mathbf{C}_{\tau r}^{\pi^{(1)}}$. By the same inclusion also (4.3) carries over, and (4.4) is preserved in view of (6.2).

In connection with (4.2) we involve the bound $\left|\pi^{(1)}-\pi\right| \leq \min \left\{C_{*} \mathbf{E}(T, 0, r, \pi)^{1 /(20 n)}, \frac{1}{2}\right\}$ (which comes from (6.1)) and the following continuity argument. We first connect ${ }^{9}$ $\pi^{(0)}:=\pi$ and $\pi^{(1)}$ by $n$-planes $\pi^{(t)}$ in $\mathcal{H}$ with $0<t<1$ such that $[0,1] \mapsto \Lambda_{n}(\mathcal{H}), t \mapsto$ $\pi^{(t)}$ is a continuous curve and such that $\left|\pi^{(t)}-\pi\right| \leq\left|\pi^{(1)}-\pi\right|$ for all $t \in[0,1]$. In particular, (6.3) is applicable when $\varpi$ is chosen as any of the $\pi^{(t)}$, and we have $\mathbf{C}_{\tau r}^{\pi^{(t)}} \cap \operatorname{spt} T_{r / 2} \subset \mathbf{C}_{2 \tau r}^{\pi}$ and $\left\langle\pi^{(t)}, \pi\right\rangle=1-\frac{1}{2}\left|\pi^{(t)}-\pi\right|^{2}>0$ for all $t \in[0,1]$. In connection with (4.1) the last inclusion implies $\left(\partial T_{r / 2}\right)\left\llcorner\mathbf{C}_{\tau r}^{\pi^{(t)}} \equiv 0\right.$, and the constancy theorem gives

$$
\left(\mathrm{p}^{\pi^{(t)}}\right)_{\sharp}\left(T_{r / 2}\left\llcorner\mathbf{C}_{\tau r}^{\pi^{(t)}}\right)=m^{(t)} \llbracket \mathbf{B}_{\tau r}^{\pi^{(t)}} \rrbracket\right.
$$

\footnotetext{
${ }^{9}$ The existence of suitable $\pi^{(t)}$ can be checked formally with the help of Lemma 2.1. Indeed, when we write $\pi=\bigwedge_{i=1}^{n} \pi_{i}$ with orthonormal $\pi_{i}$, and when $\ell: \operatorname{Span} \pi \rightarrow(\operatorname{Span} \pi)^{\perp}$ with Graph $\ell=$ $\operatorname{Span} \pi^{(1)}$ denotes the linear map of the lemma, we can set $\widetilde{\pi}^{(t)}:=\bigwedge_{i=1}^{n}\left[\pi_{i}+t \ell\left(\pi_{i}\right)\right]$ and get the $n$-planes $\pi^{(t)}:=\tilde{\pi}^{(t)} /\left|\widetilde{\pi}^{(t)}\right|$ for $0<t<1$. It can be checked that this choice of the $\pi^{(t)}$ connects $\pi$ and $\pi^{(1)}$ continuously and such that $\left|\pi^{(t)}-\pi\right|$ is increasing.
} 
for certain integers $m^{(t)}$, where $m^{(0)}$ equals $m$. We now write $\pi=\bigwedge_{i=1}^{n} \pi_{i}$ and introduce $\mathrm{p}_{i}^{\pi} \in \operatorname{Lip}(\mathcal{H})$ by $\mathrm{p}_{i}^{\pi}(z):=\left\langle\pi_{i}, z\right\rangle$ and $\varphi \in \operatorname{Lip}_{\mathrm{b}}(\mathcal{H})$ by $\varphi(z):=(\tau r-|z|)_{+}$. Since $\varphi$ vanishes outside $\mathbf{C}_{\tau r}^{\pi^{(t)}},(6.42)$ implies

$$
T_{r / 2}\left(\left(\varphi \circ \mathrm{p}^{\pi^{(t)}}\right) \bigwedge_{i=1}^{n} \mathrm{~d}\left(\mathrm{p}_{i}^{\pi} \circ \mathrm{p}^{\pi^{(t)}}\right)\right)=m^{(t)} \llbracket \mathbf{B}_{\tau r}^{\pi^{(t)}} \rrbracket\left(\varphi \bigwedge_{i=1}^{n} \mathrm{dp}_{i}^{\pi}\right)
$$

Here, by Lemma 2.2 the Lipschitz functions $\varphi \circ \mathrm{p}^{\pi^{(t)}}$ and $\mathrm{p}_{i}^{\pi} \circ \mathrm{p}^{\pi^{(t)}}$ depend continuously on $t$ (with respect to pointwise convergence in $\operatorname{Lip}(\mathcal{H})$ ), and they have uniformly bounded Lipschitz constants. Therefore, the continuity axiom and the definition of mass imply that the left hand side of (6.43) as a whole depends continuously on $t$. On the right hand side of (6.43) we rewrite with the help of (2.10)

$$
\llbracket \mathbf{B}_{\tau r}^{\pi^{(t)}} \rrbracket\left(\varphi \bigwedge_{i=1}^{n} \mathrm{dp}_{i}^{\pi}\right)=\left\langle\pi^{(t)}, \pi\right\rangle \int_{\mathbf{B}_{\tau r}^{\pi^{(t)}}}(\tau r-|z|) \mathrm{d} \mathscr{L}^{n}(z) .
$$

Here, the last expression is continuous in $t$ (the integral is in fact $t$-independent) and positive for all $t \in[0,1]$. All in all, we can conclude from (6.42) that the integers $m^{(t)}$ depend continuously on $t \in[0,1]$, and consequently we have $m^{(1)}=m^{(0)}=m$. Hence, (6.42) with $t=1$ shows that (4.2) holds also for $T_{r / 2}$ on the smaller scale $\tau r$ - with the same multiplicity $m$.

With the help of Lemma 6.2 we can iterate Proposition 6.1 in order to obtain the following decay properties of the excess.

Proposition 6.3 (excess decay). For every $\alpha \in(0,1)$ there exists a constant $\varepsilon_{2} \in$ $(0,1]$, depending only on $n, m$ and $\alpha$, with the following property. If we have $z \in \operatorname{spt} T$ and Assumption 4.1 holds for $T$ on $z+\mathbf{C}_{r}^{\pi}$ up to $\varepsilon \leq \varepsilon_{2}$, then, for all $\varrho \in(0, r]$ there is an $n$-plane $\pi_{\varrho}(z)$ with $\left|\pi_{\varrho}(z)-\pi\right| \leq \frac{1}{2}$ such that: the requirements (4.1), (4.2), and (4.3) of Assumption 4.1 hold also for $T_{r / 2}:=T\left\llcorner\left(z+\mathbf{C}_{r / 2}^{\pi}\right)\right.$ on $z+\mathbf{C}_{\varrho}^{\pi_{\varrho}(z)}$, and for some constant $C$, which depends only on $n, m$, and $\alpha$, we have

$$
\begin{gathered}
\mathbf{E}(T, z, \varrho, \pi) \leq C \mathbf{E}(T, z, r, \pi)^{3 /(20 n)}, \\
\mathbf{E}\left(T_{r / 2}, z, \varrho, \pi_{\varrho}(z)\right) \leq C\left(\frac{\varrho}{r}\right)^{2 \alpha} \mathbf{E}(T, z, r, \pi) .
\end{gathered}
$$

Proof. For ease of notation we assume $z=0$, and we abbreviate $\mathbf{E}:=\mathbf{E}(T, 0, r, \pi)$ and

$$
\beta:=\frac{3}{40 n} \text {. }
$$

We will use Proposition 6.1 with a fixed $\tau \in\left(0, \frac{1}{16}\right]$ such that $C_{*} \tau^{2} \leq \tau^{2 \alpha}$ holds for the constant $C_{*}$ of the proposition, and when we refer to (6.2) in the following we will understand that correspondingly $C_{*} \tau^{2}$ has been replaced by $\tau^{2 \alpha}$ on its right hand side. We record that this choice of $\tau$ fixes also the $\varepsilon_{1}$ of Proposition 6.1, depending only on $n, m$, and $\alpha$. We will now prove - working with some $\varepsilon_{2} \in\left(0, \varepsilon_{1}\right]$ to be chosen later depending only on the momentarily fixed quantities — the existence of a 
sequence $\left(\pi^{(i)}\right)_{i \in \mathbb{N}}$ of $n$-planes in $\mathcal{H}$ such that, understanding $\pi^{(0)}:=\pi$, the following four claims hold for all $i \in \mathbb{N}$ :

$$
\begin{gathered}
\left|\pi^{(i)}-\pi^{(i-1)}\right| \leq C_{*} \tau^{2 \beta \alpha(i-1)} \mathbf{E}^{\beta}, \\
\mathbf{C}_{\tau^{i} r}^{\varpi} \cap \operatorname{spt} T_{r / 2} \subset \mathbf{C}_{2 \tau^{i} r}^{\pi^{(i-1)}} \quad \text { whenever }|\varpi-\pi| \leq \frac{C_{*}}{1-\tau^{2 \beta \alpha}} \mathbf{E}^{\beta}, \\
\mathbf{E}\left(T_{r / 2}, 0, \tau^{i} r, \pi^{(i)}\right) \leq \tau^{2 \alpha i} \mathbf{E} .
\end{gathered}
$$$$
\text { Assumption } 4.1 \text { holds for } T_{r / 2} \text { on } \mathbf{C}_{\tau^{i} r}^{\pi^{(i)}} \text { up to } \varepsilon \text {. }
$$

Indeed, we will construct the $\pi^{(i)}$ inductively. Keeping $\varepsilon \leq \varepsilon_{2} \leq \varepsilon_{1}$ in mind, we first observe that Proposition 6.1 can be applied and gives a $\pi^{(1)}$ such that (6.44) and (6.46) hold for $i=1$. We will see at the end of the proof that we can assume

$$
\frac{1}{1-\tau^{2 \beta \alpha}} \mathbf{E}^{\beta} \leq \mathbf{E}^{1 /(20 n)},
$$

and then also the case $i=1$ of (6.45) follows from (6.3) in Proposition 6.1, and Lemma 6.2 yields $(6.47)$ for $i=1$. Now let us assume that we have found $\pi^{(1)}$, $\pi^{(2)}, \ldots, \pi^{(k)}$ up to some $k \in \mathbb{N}$ such that (6.44), (6.45), (6.46), (6.47) hold true for $i=1,2, \ldots, k$. We will then show the existence of an $n$-plane $\pi^{(k+1)}$ such that the same claims are also valid for $i=k+1$. To this end we first exploit (6.47) for $i=k$, and we apply Proposition 6.1 to $T_{r / 2}$ on $\mathbf{C}_{\tau^{k} r}^{\pi^{(k)}}$. The proposition then gives an $n$-plane $\pi^{(k+1)}$, for which, also using (6.46) for $i=k$, we have

$$
\left|\pi^{(k+1)}-\pi^{(k)}\right| \leq C_{*} \mathbf{E}\left(T_{r / 2}, 0, \tau^{k} r, \pi^{(k)}\right)^{\beta} \leq C_{*} \tau^{2 \beta \alpha k} \mathbf{E}^{\beta},
$$

and this is just (6.44) for $i=k+1$. Using (6.44) for $i=1,2, \ldots, k, k+1$ and summing the corresponding geometric series, we now observe that

$$
\left|\pi^{(i)}-\pi\right| \leq \frac{C_{*}}{1-\tau^{2 \beta \alpha}} \mathbf{E}^{\beta} \quad \text { holds for } i=1,2, \ldots, k, k+1 .
$$

In order to deal with (6.45) we consider an $n$-plane $\varpi$ with $|\varpi-\pi| \leq C_{*} \mathbf{E}^{\beta} /\left(1-\tau^{2 \beta \alpha}\right)$. By (6.49) we also infer

$$
\left|\varpi-\pi^{(k)}\right| \leq \frac{2 C_{*}}{1-\tau^{2 \beta \alpha}} \mathbf{E}^{\beta}
$$

This estimate together with (6.47) for $i=k-1$ (or with the hypotheses of the proposition, in the case $k=1$ ) will enable us to apply Lemma 5.2 for $T_{r / 2}\left\llcorner\mathbf{C}_{\tau^{k-1} r / 2}^{\pi^{(k-1)}}\right.$, once we assume

$$
\frac{2 C_{*}}{1-\tau^{2 \beta \alpha}} \mathbf{E}^{\beta} \leq \frac{\tau^{2}}{12 n} \quad \text { and } \quad \mathbf{E} \leq \lambda_{0}^{-2 n} .
$$

As (6.50) will be satisfied by our later choice of $\varepsilon_{2}$, the conclusion of Lemma 5.2 (with $\tau^{2}$ in place of $\tau$ ) indeed gives

$$
\mathbf{C}_{\tau^{k+1} r}^{\varpi} \cap \operatorname{spt}\left(T_{r / 2}\left\llcorner\mathbf{C}_{\tau^{k-1} r / 2}^{\pi^{(k-1)}}\right) \subset \mathbf{C}_{2 \tau^{k+1} r}^{\pi^{(k)}} .\right.
$$


Now we exploit the inductive assumption (6.45) for $i=k$. Decreasing the cylinder on the left hand side and increasing the one on the right hand side, this gives in particular

$$
\mathbf{C}_{\tau^{k+1} r}^{\varpi} \cap \operatorname{spt} T_{r / 2} \subset \mathbf{C}_{\tau^{k-1} r / 2}^{\pi^{(k-1)}} .
$$

By the last inclusion, (6.51) simplifies to

$$
\mathbf{C}_{\tau^{k+1} r}^{\varpi} \cap \operatorname{spt} T_{r / 2} \subset \mathbf{C}_{2 \tau^{k+1} r}^{\pi^{(k)}}
$$

so that we have obtained (6.45) for $i=k+1$. By (6.49) we can take $\varpi=\pi^{(k+1)}$, and thus we have as a particular case

$$
\mathbf{C}_{\tau^{k+1} r}^{\pi^{(k+1)}} \cap \operatorname{spt} T_{r / 2} \subset \mathbf{C}_{\tau^{k} r / 2}^{\pi^{(k)}}
$$

Next we come back to the above application of Proposition 6.1 and we observe that it also yields

$$
\mathbf{E}\left(T_{r / 2}\left\llcorner\mathbf{C}_{\tau^{k} r / 2}^{\pi^{(k)}}, 0, \tau^{k+1} r, \pi^{(k+1)}\right) \leq \tau^{2 \alpha} \mathbf{E}\left(T_{r / 2}, 0, \tau^{k} r, \pi^{(k)}\right),\right.
$$

where by (6.52) the left hand-side of the last estimate simplifies to $\mathbf{E}\left(T_{r / 2}, 0, \tau^{k+1} r, \pi^{(k+1)}\right)$. Thus, using (6.46) for $i=k$ on the right hand side, we get (6.46) also for $i=k+1$. Similarly, Lemma 6.2 together with (6.47) for $i=k$ tells us that Assumption 4.1 holds for $T_{r / 2}\left\llcorner\mathbf{C}_{\tau^{k} r / 2}^{\pi^{(k)}}\right.$ on $\mathbf{C}_{\tau^{k+1} r}^{\pi^{(k+1)}}$ up to $\varepsilon$, and by (6.52) this turns out to be the same as (6.47) for $i=k+1$. In summary, we have obtained all claims for $i=k+1$, the induction in $k$ is complete, and (6.44), (6.45), (6.46), (6.47) are now available for all $i \in \mathbb{N}$.

Now we are ready to establish the claims of the proposition. For a given $\varrho \in(0, r]$ we fix some $i \in \mathbb{N}_{0}$ with

$$
\tau^{i+1} r<\varrho \leq \tau^{i} r
$$

and we estimate via $(6.46)$

$$
\mathbf{E}\left(T_{r / 2}, 0, \varrho, \pi^{(i)}\right) \leq \tau^{-n} \mathbf{E}\left(T_{r / 2}, 0, \tau^{i} r, \pi^{(i)}\right) \leq \tau^{-n} \tau^{2 \alpha i} \mathbf{E}<\tau^{-n-2 \alpha}\left(\frac{\varrho}{r}\right)^{2 \alpha} \mathbf{E} .
$$

Here, $\tau$ is fixed, depending only on $n, m$, and $\alpha$, and thus we have obtained (6.3) with $\pi_{\varrho}(0):=\pi^{(i)}$. With this choice of $\pi_{\varrho}(0)$ the claimed validity of $(4.1),(4.2)$, and $(4.3)$ for $T_{r / 2}$ on $\mathbf{C}_{\varrho}^{\pi_{\varrho}(0)}$ is immediate from (6.47). Moreover, via (6.49) and the assumption (6.50) above, we get

$$
\left|\pi^{(i)}-\pi\right| \leq \frac{C_{*}}{1-\tau^{2 \beta \alpha}} \mathbf{E}^{\beta} \leq \frac{1}{2},
$$

which proves the stated control on $\left|\pi_{\varrho}(0)-\pi\right|$. Turning to the inequality (6.3), we first record that it is is easily verified (with constant $\tau^{-n}$ ) for $\tau r<\varrho \leq r$. For $\varrho \leq \tau r$ 
we fix as before some $i \in \mathbb{N}$ with (6.53), and we first deduce from (4.2), (2.18), and (6.45) with $\varpi=\pi$ that

$$
\begin{gathered}
\|T\|\left(\mathbf{C}_{\varrho}^{\pi}\right) \leq m \omega_{n} \varrho^{n}+\frac{1}{2} \varrho^{n} \mathbf{E}(T, 0, \varrho, \pi), \\
\mathbf{C}_{\tau^{i} r}^{\pi} \cap \operatorname{spt} T \subset \mathbf{C}_{\tau^{i-1} r}^{\pi^{(i-1)}} .
\end{gathered}
$$

Using the preceding observations along with (6.54) and (6.46), we deduce

$$
\begin{aligned}
\mathbf{E}(T, 0, \varrho, \pi) & \leq 2 \varrho^{-n}\left[\int_{\mathbf{C}_{\varrho}^{\pi}}\left|\vec{T}-\pi^{(i-1)}\right|^{2} \mathrm{~d}\|T\|+\left|\pi^{(i-1)}-\pi\right|^{2}\|T\|\left(\mathbf{C}_{\varrho}^{\pi}\right)\right] \\
& \leq 2 \varrho^{-n} \int_{\mathbf{C}_{\tau^{i} r}^{\pi}}\left|\vec{T}-\pi^{(i-1)}\right|^{2} \mathrm{~d}\left\|T_{r / 2}\right\|+\frac{C_{*}^{2}}{\left(1-\tau^{2 \beta \alpha}\right)^{2}} \mathbf{E}^{2 \beta}\left[2 m \omega_{n}+\mathbf{E}(T, 0, \varrho, \pi)\right] \\
& \leq 2 \tau^{-2 n} \mathbf{E}\left(T_{r / 2}, 0, \tau^{i-1} r, \pi^{(i-1)}\right)+\frac{2 m \omega_{n} C_{*}^{2}}{\left(1-\tau^{2 \beta \alpha}\right)^{2}} \mathbf{E}^{2 \beta}+\frac{1}{4} \mathbf{E}(T, 0, \varrho, \pi) \\
& \leq 2 \tau^{-2 n} \mathbf{E}+\frac{2 m \omega_{n} C_{*}^{2}}{\left(1-\tau^{2 \beta \alpha}\right)^{2}} \mathbf{E}^{2 \beta}+\frac{1}{4} \mathbf{E}(T, 0, \varrho, \pi),
\end{aligned}
$$

and absorbing the last term, we get (6.3) also in the remaining cases. To conclude the proof it suffices to take $\varepsilon_{2} \in\left(0, \varepsilon_{1}\right]$ small enough that $\mathbf{E} \leq \varepsilon_{2}$ implies the previously exploited smallness conditions (6.48) and (6.50). Recalling the choices made at the beginning of the proof (in connection with (6.48) note specifically $\beta=3 /(40 n)>$ $1 /(20 n))$, such an $\varepsilon_{2}$ can be chosen, depending only on $n, m$, and $\alpha$.

Proof of Theorem 4.2. Highlighting their dependence on $\alpha$, we write $\varepsilon_{2 ; \alpha}$ and $C_{\alpha}$ for the constants of Proposition 6.3. Then we choose

$$
\varepsilon_{*}:=\min \left\{\varepsilon_{0}, 2^{-n}\left(C_{1 / 2}^{-1} \gamma\right)^{20 n / 3}, 2^{-n} \varepsilon_{2 ; 1 / 2}\right\}
$$

where $\varepsilon_{0}$ is the constant of Lemma 5.1 and $\gamma$ the one of Lemma 5.5. In the situation of Theorem 4.2 we can then apply Lemma 5.3 with $\lambda=1$, and we write $f: \mathbf{B}_{r / 2}^{\pi} \rightarrow(\operatorname{Span} \pi)^{\perp}$ for the Lipschitz function of the lemma with $\operatorname{Lip}(f) \leq 1$. Moreover, observing first $\mathbf{E}(T, z, r / 2, \pi) \leq 2^{n} \mathbf{E}(T, 0, r, \pi) \leq \min \left\{\left(C_{1 / 2}^{-1} \gamma\right)^{20 n / 3}, \varepsilon_{2 ; 1 / 2}\right\}$ for all $z \in \mathbf{C}_{r / 2}^{\pi}$, we can apply Proposition 6.3 with $\alpha=\frac{1}{2}$ on the cylinders $z+\mathbf{C}_{r / 2}^{\pi}$ to get

$$
\begin{gathered}
\mathbf{E}(T, z, \varrho, \pi) \leq C_{1 / 2} \mathbf{E}(T, z, r / 2, \pi)^{3 /(20 n)} \leq \gamma \\
\mathbf{E}\left(T \mathrm{~L}\left(z+\mathbf{C}_{r / 4}^{\pi}\right), z, \varrho, \widetilde{\pi}_{\varrho}(z)\right) \leq C_{1 / 2} \frac{\varrho}{r} \mathbf{E}(T, z, r / 2, \pi) \leq C_{1 / 2} \frac{\varrho}{r} \varepsilon_{2 ; 1 / 2}
\end{gathered}
$$

for all $z \in \operatorname{spt} T_{r / 2}$ and $0<\varrho \leq r / 2$. Here, the $\tilde{\pi}_{\varrho}(z)$ are the $n$-planes given by Proposition 6.3 , and we have once more abbreviated $T_{r / 2}:=T\left\llcorner\mathrm{C}_{r / 2}^{\pi}\right.$. We now make use of the fact that spt $T_{r / 2}$ is compact by the discussion at the very end of Section 2.4. It follows that $\mathrm{p}^{\pi}\left(\operatorname{spt} T_{r / 2}\right)$ is closed, and this together with (4.2) ensures $\mathbf{B}_{r / 2}^{\pi} \subset \mathrm{p}^{\pi}\left(\operatorname{spt} T_{r / 2}\right)$. As $\mathbf{E}(T, z, \varrho, \pi)$ remains unchanged when one adds a vector from 
$(\operatorname{Span} \pi)^{\perp},(6.55)$ implies that the set $G_{1}$ from Lemma 5.5 is all of $\mathbf{B}_{r / 2}^{\pi}$, and then (5.8) gives

$$
T_{r / 2}=m \llbracket \text { Graph } f \rrbracket .
$$

For every given $\alpha \in(0,1)$ we next fix $r_{\alpha} \in(0, r / 2]$ small enough that $C_{1 / 2} \frac{r_{\alpha}}{r} \varepsilon_{2 ; 1 / 2} \leq$ $\varepsilon_{2 ; \alpha}$ holds. Coming back to (6.56) we then infer $\mathbf{E}\left(T\left\llcorner\left(z+\mathbf{C}_{r / 4}^{\pi}\right), z, r_{\alpha}, \widetilde{\pi}_{r_{\alpha}}(z)\right) \leq \varepsilon_{2 ; \alpha}\right.$, and hence - as (4.1), (4.2), (4.3) are already guaranteed by the preceding application of Proposition 6.3 - Assumption 4.1 holds for $T\left\llcorner\left(z+\mathbf{C}_{r / 4}^{\pi}\right)\right.$ on $z+\mathbf{C}_{r_{\alpha}}^{\widetilde{\pi}_{r_{\alpha}}(z)}$ up to $\varepsilon_{2 ; \alpha}$, for all $z \in \operatorname{spt} T_{r / 2}=$ Graph $f$. We can therefore apply Proposition 6.3 again, and decreasing the domain of integration on the left hand side from a cylinder to a ball we deduce

$$
\varrho^{-n} \int_{F(x)+\mathbf{B}_{\varrho}}\left|\vec{T}-\pi_{\varrho}(x)\right|^{2} \mathrm{~d}\|T\| \leq C_{\alpha}\left(\frac{\varrho}{r_{\alpha}}\right)^{2 \alpha} \varepsilon_{2 ; \alpha} .
$$

for all $x \in \mathbf{B}_{r / 2}^{\pi}$ and all $\varrho \leq r_{\alpha} / 2 \leq r / 4$ with some $n$-planes $\pi_{\varrho}(x)$ such that $\left|\pi_{\varrho}(x)-\pi\right| \leq \frac{1}{2}$. By Lemma 2.1 we can find linear maps $\ell_{\varrho}(x): \operatorname{Span} \pi \rightarrow(\operatorname{Span} \pi)^{\perp}$ with $\operatorname{Graph} \ell_{\varrho}(x)=\operatorname{Span} \pi_{\varrho}(x)$ and $\left|\ell_{\varrho}(x)\right| \leq 1$. As earlier we write $F$ and $L_{\varrho}(x)$ for the graph mappings of $f$ and $\ell_{\varrho}(x)$, respectively, and we observe $\pi_{\varrho}(x)=\frac{\bigwedge_{i=1}^{n} L_{\varrho}(x) \pi_{i}}{\mathrm{~J}_{n}\left(L_{\varrho}(x)\right)}$ (compare, for instance, the proof of Lemma 2.1 for the identification of the right sign). In the next step we exploit that $|\mathrm{D} f|$ and $\mathrm{J}_{n}\left(L_{\varrho}(x)\right)$ are bounded by a dimensional constant, and we make use of the equalities $1=|\pi|=\left|[\mathrm{D} f]_{0}^{\pi}\right|=\left|\left[\ell_{\varrho}(x)\right]_{0}^{\pi}\right|$ and $\left|\mathrm{D} f-\ell_{\varrho}(x)\right|=\left|\left[\mathrm{D} f-\ell_{\varrho}(x)\right]_{1}^{\pi}\right|=\left|[\mathrm{D} f]_{1}^{\pi}-\left[\ell_{\varrho}(x)\right]_{1}^{\pi}\right|$ in the terminology of Section 2.5. Employing also $(2.24)$ and $(2.12), \mathscr{L}^{n}$-a. e. on $\mathbf{B}_{r / 2}^{\pi}$ we can estimate, with a dimensional constant,

$$
\begin{aligned}
\left|\mathrm{D} f-\ell_{\varrho}(x)\right|^{2} & \leq C\left|\frac{\mathrm{D} f}{\mathrm{~J}_{n}\left(L_{\varrho}(x)\right)}-\frac{\ell_{\varrho}(x)}{\mathrm{J}_{n}\left(L_{\varrho}(x)\right)}\right|^{2} \\
& \leq C\left[\left|\frac{1}{\mathrm{~J}_{n}\left(L_{\varrho}(x)\right)}-\frac{1}{\mathrm{~J}_{n}(\mathrm{D} F)}\right|^{2}+\left|\frac{\mathrm{D} f}{\mathrm{~J}_{n}(\mathrm{D} F)}-\frac{\ell_{\varrho}(x)}{\mathrm{J}_{n}\left(L_{\varrho}(x)\right)}\right|^{2}\right] \\
& \leq C \sum_{k=0}^{n}\left|\frac{[\mathrm{D} f]_{k}^{\pi}}{\mathrm{J}_{n}(\mathrm{D} F)}-\frac{\left[\ell_{\varrho}(x)\right]_{k}^{\pi}}{\mathrm{J}_{n}\left(L_{\varrho}(x)\right)}\right|^{2} \\
& =C\left|\frac{\bigwedge_{i=1}^{n}(\mathrm{D} F) \pi_{i}}{\mathrm{~J}_{n}(\mathrm{D} F)}-\frac{\bigwedge_{i=1}^{n} L_{\varrho}(x) \pi_{i}}{\mathrm{~J}_{n}\left(L_{\varrho}(x)\right)}\right|^{2} \\
& =C\left|\vec{T} \circ F-\pi_{\varrho}(x)\right|^{2} .
\end{aligned}
$$

When we use the last estimate, (2.13), the area formula (2.4) (where the Jacobian is estimated from below by 1$)$, and the inclusion $F\left(x+\mathbf{B}_{\varrho / 2}^{\pi}\right) \subset F(x)+\mathbf{B}_{\varrho}$ in (6.57), we find $^{10}$

$$
\varrho^{-n} \int_{x+\mathbf{B}_{\varrho / 2}^{\pi}}\left|\mathrm{D} f-\ell_{\varrho}(x)\right|^{2} \mathrm{~d} \mathscr{L}^{n} \leq C C_{\alpha}\left(\frac{\varrho}{r_{\alpha}}\right)^{2 \alpha} .
$$

\footnotetext{
${ }^{10}$ Note that here the linear map $\ell_{\varrho}(x)$ is understood as constant in the integration variable.
} 
for all $x \in \mathbf{B}_{r / 2}^{\pi}$ and $\varrho \in\left(0, r_{\alpha} / 2\right]$. From the last estimate it is straightforward to conclude that for every fixed $x \in \mathbf{B}_{r / 2}^{\pi}$ the sequence $\left(\ell_{2^{-k} r_{\alpha}}(x)\right)_{k \in \mathbb{N}}$ is a Cauchy sequence which converges to some $\ell(x)$ with error bound $\left|\ell_{2^{-k} r_{\alpha}}(x)-\ell(x)\right| \leq C 2^{-k \alpha}$, so that we get

$$
\varrho^{-n} \int_{x+\mathbf{B}_{\varrho}^{\pi}}|\mathrm{D} f-\ell(x)|^{2} \mathrm{~d} \mathscr{L}^{n} \leq \widetilde{C}_{\alpha}\left(\frac{\varrho}{r_{\alpha}}\right)^{2 \alpha}
$$

for all $x \in \mathbf{B}_{r / 2}^{\pi}$ and $\varrho \in\left(0, r_{\alpha} / 4\right]$, with yet another constant $\widetilde{C}_{\alpha}$, which depends only on $n, m$, and $\alpha$. For every Lebesgue point $x$ of $\mathrm{D} f$ the corresponding Lebesgue value is $\ell(x)$, thus $\ell$ is a representative of $\mathrm{D} f$, and for all $x, \widetilde{x} \in \mathbf{B}_{r / 2}^{\pi}$ with $0 \neq|\widetilde{x}-x| \leq r_{\alpha} / 4$ we have

$$
\begin{aligned}
|\ell(\widetilde{x})-\ell(x)|^{2} & \leq \frac{1}{\omega_{n}(|\widetilde{x}-x| / 2)^{n}} \int_{\frac{x+\widetilde{x}}{2}+\mathbf{B}_{|\widetilde{x}-x| / 2}^{\pi}}\left[|\mathrm{D} f-\ell(x)|^{2}+|\mathrm{D} f-\ell(\widetilde{x})|^{2}\right] \mathrm{d} \mathscr{L}^{n} \\
& \leq \frac{2^{n}}{\omega_{n}}|\widetilde{x}-x|^{-n}\left[\int_{x+\mathbf{B}_{|\widetilde{x}-x|}^{\pi}}|\mathrm{D} f-\ell(x)|^{2} \mathrm{~d} \mathscr{L}^{n}+\int_{\widetilde{x}^{n}+\mathbf{B}_{|\widetilde{x}-x|}^{\pi}}|\mathrm{D} f-\ell(\widetilde{x})|^{2} \mathrm{~d} \mathscr{L}^{n}\right] \\
& \leq \frac{2^{n+1}}{\omega_{n}} \widetilde{C}_{\alpha}\left(\frac{|\widetilde{x}-x|}{r_{\alpha}}\right)^{2 \alpha} .
\end{aligned}
$$

This proves that the representative $\ell$ of $\mathrm{D} f$ is $\alpha$-Hölder continuous on $\mathbf{B}_{r / 2}^{\pi}$ for all $\alpha \in(0,1)$, and now it is easy to check that $f$ is Fréchet differentiable at every $x \in \mathbf{B}_{r / 2}^{\pi}$ with derivative $\mathrm{D} f(x)=\ell(x)$. This ends the proof of Theorem 4.2.

\section{Partial Regularity}

In this section we prove Theorem 1.2. We begin with the following lemma, that eventually will be applied with $\mu=\|T\|$ and $\Theta$ equal to the spherical $n$-dimensional density of $\mu$.

Lemma 7.1. Consider a finite, non-negative Borel measure $\mu$ in $\mathcal{H}$, a relatively open subset $\Gamma$ of spt $\mu$, and an upper semicontinuous function $\Theta: \Gamma \rightarrow[0, \infty)$. If one has $\Theta \in \mathbb{N} \mu$-a. e., then the set

$$
D:=\{z \in \Gamma: \Theta(z) \in \mathbb{N} \text { and } \Theta \equiv \Theta(z) \mu \text {-a. e. in a neighborhood of } z\}
$$

is dense in $\Gamma$.

Proof. Let $\widetilde{\Gamma}:=\Theta^{-1}(\mathbb{N})$, fix $z_{0} \in \Gamma$ and $r>0$, and observe that $\left(z_{0}+\mathbf{B}_{r}\right) \cap \widetilde{\Gamma}$ has positive measure and is in particular not empty. Next consider a minimum point $w_{0}$ for the restriction of $\Theta$ to $\left(z_{0}+\mathbf{B}_{r}\right) \cap \widetilde{\Gamma}$. Clearly, this minimum point exists, because there are finitely many integers less than $\Theta\left(z_{0}\right)$. Then we have

$$
\Theta\left(w_{0}\right) \leq \Theta(z) \quad \forall z \in\left(z_{0}+\mathbf{B}_{r}\right) \cap \widetilde{\Gamma}
$$

and, by the upper semicontinuity of $\Theta$, we get $\Theta \equiv \Theta\left(w_{0}\right)$ on $\left(w_{0}+\mathbf{B}_{r^{\prime}}\right) \cap \widetilde{\Gamma}$ for some $r^{\prime}>0$. Therefore $w_{0} \in D$ and, as $z_{0}$ and $r$ are arbitrary, this proves the density of $D$. 
Proof of Theorem 1.2. Applying the previous lemma with $\mu=\|T\|, \Gamma=\Omega \cap \operatorname{spt} T$, and $\Theta=\Theta^{n}(\|T\|, \cdot)$, we know that the set $D_{1}$ of points $z \in \Omega \cap$ spt $T$ such that $\Theta(z) \in \mathbb{N}$ and $\Theta=\Theta(z) \mathscr{H}^{n}$-a. e. in a neighborhood of $z$ is dense. Let now $\varepsilon>0$. Consider the set $D_{2}$ of points $z \in D_{1}$ such that $z+\mathbf{B}_{r}^{\pi}+\mathbf{B}_{r}^{\perp \pi}$ is at positive distance from $\mathcal{H} \backslash \Omega$,

$$
\begin{gathered}
\Theta \equiv m \quad\|T\| \text {-a. e. on } z+\mathbf{B}_{r}^{\pi}+\mathbf{B}_{r}^{\perp \pi}, \quad \int_{z+\mathbf{B}_{r}^{\pi}+\mathbf{B}_{r}^{\perp \pi}}|T-\pi|^{2} \mathrm{~d}\|T\|<\varepsilon r^{n}, \\
\|T\|\left(z+\mathbf{B}_{2 r}^{\pi}+\left(\mathbf{B}_{2 r}^{\perp \pi} \backslash \mathbf{B}_{r / 4}^{\perp \pi}\right)\right)<\varepsilon \omega_{n} r^{n} \\
\left(m-\frac{1}{2}\right) \omega_{n} r^{n}<\|T\|\left(z+\mathbf{B}_{r}^{\pi}+\mathbf{B}_{r}^{\perp \pi}\right)<\left(m+\frac{1}{2}\right) \omega_{n} r^{n},
\end{gathered}
$$

for some $m \in \mathbb{N}$, some $r>0$, and some $n$-plane $\pi$. From the definitions of $D_{1}$ and $D_{2}$ and from Proposition B.2 we infer that every point in $D_{1}$ has a neighborhood $\mathcal{N}$ with $\|T\|\left(\mathcal{N} \backslash D_{2}\right)=0$. Hence we know that also $D_{2}$ is dense in $\Omega \cap \operatorname{spt} T$.

Let $z \in D_{2}$ and $m, r, \pi$ be chosen according to (7.1), (7.2), (7.3). Moreover set $U:=z+\mathbf{B}_{r}^{\pi}+\mathbf{B}_{r}^{\perp \pi}$. We claim that $\widetilde{T}:=T\llcorner U$ fulfills, possibly with opposite orientation, the assumptions of the $\varepsilon$-regularity Theorem 4.2, provided that we choose $\varepsilon$ sufficiently small depending only on $n$ and $m$ (notice that since we are working inside $D_{1}$ we can neglect the dependence on $m$ ). In order to prove the claim, we show first by contradiction that

$$
U \cap \operatorname{spt} T \subset z+\mathbf{B}_{r}^{\pi}+\mathbf{B}_{r / 2}^{\perp \pi} .
$$

If $w \in U \cap \operatorname{spt} T$ satisfies $\left|q^{\pi}(w-z)\right| \geq r / 2$, the ball $w+\mathbf{B}_{r / 4}$ is contained in $z+\mathbf{B}_{2 r}^{\pi}+\left(\mathbf{B}_{2 r}^{\perp \pi} \backslash \mathbf{B}_{r / 4}^{\perp \pi}\right)$, hence (7.2) yields $\|T\|\left(w+\mathbf{B}_{r / 4}\right)<\varepsilon \omega^{n} r^{n}$. This contradicts (2.20) if $\varepsilon<4^{-n}$. From (7.4) and the assumption $(\partial T)\llcorner\Omega \equiv 0$ we obtain that $\widetilde{T}$ has no boundary in the cylinder $z+\mathbf{C}_{r}^{\pi}$. Then, the constancy theorem gives $\left(\mathrm{p}^{\pi}\right)_{\sharp}\left(T \mathrm{~L}\left(z+\mathbf{C}_{r}^{\pi}\right)\right)=m^{\prime} \llbracket z+\mathbf{B}_{r}^{\pi} \rrbracket$ for some integer $m^{\prime}$, and (7.3) together with (2.18) yields $\left|m^{\prime}\right|=m$, if $\varepsilon<1$.

Therefore either $\widetilde{T}$ or $-\widetilde{T}$ satisfy the four conditions listed in Assumption 4.1. Moreover, a simple retraction argument shows that $\widetilde{T}$ (and $-\widetilde{T}$ as well) is locally minimizing in $z+\mathbf{C}_{r}^{\pi}$. Indeed, consider $R \in \mathbf{I}_{n}(\mathcal{H})$ with $\partial R \equiv 0$ and spt $R \subset z+\mathbf{C}_{r}^{\pi}$, and let us prove that $\mathbf{M}(\widetilde{T}) \leq \mathbf{M}(\widetilde{T}+R)$. First, possibly using a retraction of the cylinder on $\bar{U}$, we can assume that spt $R \subset \bar{U}$. Then, recalling that $\bar{U}$ has positive distance from $\mathcal{H} \backslash \Omega$, we can use the local minimality of $T$ in $\Omega$ to compare $T$ with $T+R$ and get $\mathbf{M}(T) \leq \mathbf{M}(T+R) \leq \mathbf{M}(\widetilde{T}+R)+\|T\|(\mathcal{H} \backslash U)$. Subtracting $\|T\|(\mathcal{H} \backslash U)$ from both sides, we arrive at $\mathbf{M}(\widetilde{T}) \leq \mathbf{M}(\widetilde{T}+R)$ as claimed.

Finally, notice that possibly choosing $r$ slightly smaller we can retain the same properties above of $\widetilde{T}$ and have also $\widetilde{T} \in \mathbf{I}_{n}(\mathcal{H})$. Choosing also $\varepsilon \leq \varepsilon_{*}$ in our construction of $\widetilde{T}$ we can therefore apply Theorem 4.2 to obtain that $U$ consists of regular points of $\widetilde{T}$ only, and the same holds for $T$. Since $z \in D_{2}$ is arbitrary, this proves that $\operatorname{Reg} T$ is dense. 


\section{Appendix A. Cartesian products and homotopy COnstruction}

In the following we will be concerned with Cartesian products of currents, which arise by a slight variant of the cone construction as presented in [5, Definition 10.1] (compare also with the classical construction in [20]), and we will work in the Hilbert space $\mathbb{R} \times \mathcal{H}$ (with inner product $\left.\left\langle\left(t_{1}, z_{1}\right),\left(t_{2}, z_{2}\right)\right\rangle:=t_{1} t_{2}+\left\langle z_{1}, z_{2}\right\rangle\right)$. In this regard we use the isometric embeddings

$$
\mathrm{i}_{t}: \mathcal{H} \rightarrow \mathbb{R} \times \mathcal{H}, \quad \text { given by } \mathrm{i}_{t}(z):=(t, z),
$$

and for a Lipschitz function $\chi: \mathbb{R} \times \mathcal{H} \rightarrow \mathbb{R}$ we write $\mathrm{D}_{t} \chi$ for its partial derivative with respect to the first variable. Given any $\sigma$-finite Borel measure $\mu$ on $\mathcal{H}$ this derivative exists $\left(\mathscr{L}^{1} \otimes \mu\right)$-a. e. in $\mathbb{R} \times \mathcal{H}$ and is bounded by $\operatorname{Lip}(\chi)$. Consequently, for $\mathscr{L}^{1}$-a. e. $t \in \mathbb{R}$ the composition $\left(\mathrm{D}_{t} \chi\right) \circ \mathrm{i}_{t}$ is defined $\mu$-almost-everywhere. This last observation, applied with $\chi=\psi_{j}$ and $\mu=\|T\|$, makes the following definition well-posed:

Lemma A.1 (Cartesian products). Consider an $(n-1)$-current $T \in \mathbf{M}_{n-1}(\mathcal{H})$. Then the specification

$$
(\llbracket 0,1 \rrbracket \times T)\left(\varphi \bigwedge_{i=1}^{n} \mathrm{~d} \psi_{i}\right):=\sum_{j=1}^{n}(-1)^{j-1} \int_{0}^{1}\left(\left(\mathrm{i}_{t}\right)_{\sharp} T\right)\left(\varphi \mathrm{D}_{t} \psi_{j} \bigwedge_{\substack{i=1 \\ i \neq j}}^{n} \mathrm{~d} \psi_{i}\right) \mathrm{d} \mathscr{L}^{1}(t)
$$

for $(\varphi, \psi) \in \operatorname{Lip}_{\mathrm{b}}(\mathbb{R} \times \mathcal{H}) \times \operatorname{Lip}(\mathbb{R} \times \mathcal{H})^{n}$ defines a metric functional $T$ with

$$
\|\llbracket 0,1 \rrbracket \times T\| \leq n\left(\mathscr{L}^{1}\llcorner[0,1]) \otimes\|T\| .\right.
$$

Moreover:

- if $T \in \mathbf{N}_{n-1}(\mathcal{H})$ is normal, then $\llbracket 0,1 \rrbracket \times T \in \mathbf{N}_{n}(\mathbb{R} \times \mathcal{H})$ is a normal $n$ dimensional current with

$$
\partial(\llbracket 0,1 \rrbracket \times T)=\left(\mathrm{i}_{1}\right)_{\sharp} T-\left(\mathrm{i}_{0}\right)_{\sharp} T-\llbracket 0,1 \rrbracket \times(\partial T) ;
$$

- if $T$ is (integer-)rectifiable, then also $\llbracket 0,1 \rrbracket \times T$ is an (integer-)rectifiable current.

Proof. Clearly, $\llbracket 0,1 \rrbracket \times T$ is linear in $\varphi$ and each $\psi_{i}$ and is thus a metric functional. To prove $(\mathrm{A} .1)$ we consider $(\varphi, \psi) \in \operatorname{Lip}_{\mathrm{b}}(\mathbb{R} \times \mathcal{H}) \times \operatorname{Lip}(\mathbb{R} \times \mathcal{H})^{n}$ with $\operatorname{Lip}\left(\psi_{i}\right) \leq 1$ for all $i \in\{1,2, \ldots, n\}$. Then from the definitions of the Cartesian product, the pushforward, and the mass, combined with the Lipschitz bound $\operatorname{Lip}\left(\psi_{i} \circ \mathrm{o}_{t}\right) \leq 1$, we readily deduce

$$
\left|(\llbracket 0,1 \rrbracket \times T)\left(\varphi \bigwedge_{i=1}^{n} \mathrm{~d} \psi_{i}\right)\right| \leq n \int_{0}^{1} \int_{\mathcal{H}}\left|\varphi \circ \mathrm{i}_{t}\right| \mathrm{d}\|T\| \mathrm{d} \mathscr{L}^{1}(t)=n \int_{[0,1] \times \mathcal{H}}|\varphi| \mathrm{d}\left(\mathscr{L}^{1} \otimes\|T\|\right) .
$$

By multilinearity and again by the definition of mass this implies (A.1) and in particular $\mathbf{M}(\llbracket 0,1 \rrbracket \times T)<\infty$.

If $T$ is a normal current, following the proof of [5, Proposition 10.2] one can show that $\llbracket 0,1 \rrbracket \times T$ satisfies the continuity axiom and the formula (A.2). Indeed, we will not 
discuss the adaption of the respective arguments, as the required changes ${ }^{11}$ are mostly notational ones. By applying (A.1) with $\partial T$ in place of $T$ we obtain $\mathbf{M}(\llbracket 0,1 \rrbracket \times \partial T)<$ $\infty$; then (A.2) allows to conclude $\mathbf{M}(\partial(\llbracket 0,1 \rrbracket \times T))<\infty$. As the locality axiom is easily verified, we thus have $\llbracket 0,1 \rrbracket \times T \in \mathbf{N}_{n}(\mathbb{R} \times \mathcal{H})$.

Finally, for $T \in \mathscr{R}_{n-1}(\mathcal{H})$ we denote by $\left(\mathrm{S}_{T}, \theta_{T}, \vec{T}\right)$ a corresponding triplet as in Section 2.4 with countably $(n-1)$-rectifiable $\mathrm{S}_{T}$, and we write $\vec{T}=\bigwedge_{i=1}^{n-1} T_{i}$ with Borel functions $T_{i}: \mathrm{S}_{T} \rightarrow \mathcal{H}$. As candidates for the multiplicity and the orientation of $\llbracket 0,1 \rrbracket \times T$ we consider $\vartheta:[0,1] \times \mathrm{S}_{T} \rightarrow(0, \infty)$ with $\vartheta(t, z):=\theta_{T}(z)$ and $\tau:[0,1] \times \mathrm{S}_{T} \rightarrow \Lambda_{n}(\mathbb{R} \times \mathcal{H})$ with $\tau(t, z):=(1,0) \wedge \bigwedge_{i=1}^{n-1}\left(0, T_{i}(z)\right)$. We moreover fix $(\varphi, \psi) \in \operatorname{Lip}_{\mathrm{b}}(\mathbb{R} \times \mathcal{H}) \times \operatorname{Lip}(\mathbb{R} \times \mathcal{H})^{n}$, and we recall that the inner product of $n$-vectors is given by the determinant in (2.1); by Laplace expansion of this determinant we then get

$$
\begin{aligned}
& \left\langle\bigwedge_{i=1}^{n} \mathrm{D}^{[0,1] \times \mathrm{S}_{T}} \psi_{i}(t, z), \tau(t, z)\right\rangle \\
& \quad=\sum_{j=1}^{n}(-1)^{j-1}\left\langle\mathrm{D}^{[0,1] \times \mathrm{S}_{T}} \psi_{j}(t, z),(1,0)\right\rangle\left\langle\bigwedge_{\substack{i=1 \\
i \neq j}}^{n} \mathrm{D}^{[0,1] \times \mathrm{S}_{T}} \psi_{i}(t, z), \bigwedge_{i=1}^{n-1}\left(0, T_{i}(z)\right)\right\rangle \\
& \quad=\sum_{j=1}^{n}(-1)^{j-1} \mathrm{D}_{t} \psi_{j}(t, z)\left\langle\bigwedge_{\substack{i=1 \\
i \neq j}}^{n} \mathrm{D}^{\mathrm{S}_{T}}\left(\psi_{i} \circ \mathrm{i}_{t}\right)(z), \vec{T}(z)\right\rangle
\end{aligned}
$$

for all $(t, z) \in[0,1] \times \mathrm{S}_{T}$. We multiply this equality with $\varphi(t, z)$ and $\vartheta(t, z)=\theta_{T}(z)$ and integrate with respect to $\mathscr{H}^{n-1}$ in $z$. Using additionally the representation $(2.10)$ for the $(n-1)$-current $T$ we infer

$$
\begin{aligned}
\int_{\{t\} \times \mathrm{S}_{T}} \varphi\left\langle\bigwedge_{i=1}^{n} \mathrm{D}^{[0,1] \times \mathrm{S}_{T}} \psi_{i}, \tau\right\rangle \vartheta \mathrm{d} \mathscr{H}^{n-1} & \\
& =\sum_{j=1}^{n}(-1)^{j-1} \int_{\mathrm{S}_{T}}\left(\varphi \circ \mathrm{i}_{t}\right)\left(\mathrm{D}_{t} \psi_{j} \circ \mathrm{i}_{t}\right)\left\langle\bigwedge_{\substack{i=1 \\
i \neq j}}^{n} \mathrm{D}^{\mathrm{S}_{T}}\left(\psi_{i} \circ \mathrm{i}_{t}\right), \vec{T}\right\rangle \theta_{T} \mathrm{~d} \mathscr{H}^{n-1} \\
& =\sum_{j=1}^{n}(-1)^{j-1}\left(\left(\mathrm{i}_{t}\right)_{\sharp} T\right)\left(\varphi \mathrm{D}_{t} \psi_{j} \bigwedge_{\substack{i=1 \\
i \neq j}}^{n} \mathrm{~d} \psi_{i}\right)
\end{aligned}
$$

for all $t \in[0,1]$. Now we integrate the resulting equality also in $t$, on the left hand side we involve the coarea formula [6, Theorem 9.4] with area factor 1 on the countably

\footnotetext{
${ }^{11}$ Indeed, $t$ does now denote an extra variable instead of a radial one, and the partial functions $\varphi \circ \mathrm{i}_{t}$ and $\psi \circ \mathrm{i}_{t}$ replace $\varphi_{t}$ and $\pi_{t}$. Otherwise the main difference is that $S\left(\varphi_{0} \mathrm{~d} \pi_{0}\right)$ vanishes in [5, Proposition 10.2], while in our setup the corresponding term $\left(\left(\mathrm{i}_{0}\right)_{\sharp}\right) T\left(\varphi \bigwedge_{i=1}^{n} \mathrm{~d} \psi_{i}\right)$ remains on the right hand side of (A.2).
} 
$n$-rectifiable set $[0,1] \times \mathrm{S}_{T}$, and on the right hand side we exploit the definition of $\llbracket 0,1 \rrbracket \times T$. We then find

$$
\int_{[0,1] \times \mathrm{S}_{T}} \varphi\left\langle\bigwedge_{i=1}^{n} \mathrm{D}^{[0,1] \times \mathrm{S}_{T}} \psi_{i}, \tau\right\rangle \vartheta \mathrm{d} \mathscr{H}^{n}=(\llbracket 0,1 \rrbracket \times T)\left(\varphi \bigwedge_{i=1}^{n} \mathrm{~d} \psi_{i}\right) .
$$

All in all, we have thus shown that $\llbracket 0,1 \rrbracket \times T$ is the current which is induced by the triplet $\left([0,1] \times \mathrm{S}_{T}, \vartheta, \tau\right)$ in the sense of $(2.10)$. By [5, Theorem 9.1] this current is rectifiable.

Finally, for $T \in \mathrm{I}_{n-1}(\mathcal{H})$ the multiplicity $\theta_{T}$ can be chosen $\mathbb{N}$-valued. Consequently, the above function $\vartheta$ is $\mathbb{N}$-valued, and in conclusion we get $\llbracket 0,1 \rrbracket \times T \in \mathrm{I}_{n}(\mathbb{R} \times \mathcal{H})$ in this situation.

Remark A.2. The following two observations concern the rectifiability part of the preceding argument. They are scarcely relevant for our purposes, but may still be worth pointing out:

- For rectifiable currents $T$ one can actually improve the estimate (A.1) - by similar arguments as above and the representation of mass in (2.13) - to the equality

$$
\|\llbracket 0,1 \rrbracket \times T\|=\left(\mathscr{L}^{1}\llcorner[0,1]) \otimes\|T\| .\right.
$$

This situation may be compared to [20, 2.10.45] and [20, 3.2.23].

- The rectifiability of $\llbracket 0,1 \rrbracket \times T$ can alternatively be proved by the following shorter and more elementary reasoning provided that $T$ is normal and rectifiable: By definition $T$ is concentrated on a countably $(n-1)$-rectifiable set $S$, and by (A.1) $\llbracket 0,1 \rrbracket \times T$ is then concentrated on the countably $n$-rectifiable set $[0,1] \times S$. By $[5 \text {, Theorem 3.9 }]^{12}$ this property already implies the rectifiability of $\llbracket 0,1 \rrbracket \times T$.

We now consider the pushforward $H_{\sharp}(\llbracket 0,1 \rrbracket \times T)$ under a homotopy $H:[0,1] \times \mathcal{H} \rightarrow$ $\mathcal{H}$. We remark that for $H(t, z)=t z+(1-t) z_{0}$ this pushforward becomes a cone over $T$ with vertex $z_{0} \in \mathcal{H}$, and we get back the original cone construction of $[5$, Definition 10.1] as a special case. However, in the following we will make a different choice of $H$, which will lead to a proof of the following lemma.

Lemma A.3 (homotopy retraction on a graph). Consider a current $T \in \mathbf{N}_{n-1}(\mathcal{H})$ with $\partial T \equiv 0$, an $n$-plane $\pi$ in $\mathcal{H}$, and a Lipschitz function $f: \mathrm{p}^{\pi}(\operatorname{spt} T) \rightarrow(\operatorname{Span} \pi)^{\perp}$. If we have

$$
K:=\sup _{\operatorname{spt} T}\left|\mathrm{q}^{\pi}-f \circ \mathrm{p}^{\pi}\right|<\infty
$$

then there exists a another current $V \in \mathbf{N}_{n}(\mathcal{H})$ with $\operatorname{spt} V \subset\left(\mathrm{p}^{\pi}\right)^{-1}\left(\mathrm{p}^{\pi}(\operatorname{spt} T)\right)$, (A.5) $\partial V=T-F_{\sharp}\left(\mathrm{p}^{\pi}\right)_{\sharp} T \quad$ and $\quad \mathbf{M}(V) \leq n K(1+\operatorname{Lip}(f))^{n-1}\|T\|(\mathcal{H} \backslash \operatorname{Graph} f)$. Additionally, if $T$ is (integer-)rectifiable, then also $V$ can be chosen (integer-)rectifiable.

\footnotetext{
${ }^{12}$ The application of this theorem relies on the fact that $\llbracket 0,1 \rrbracket \times T$ is normal by the preceding reasoning.
} 
Proof. We set

$$
V=H_{\sharp}(\llbracket 0,1 \rrbracket \times T),
$$

where $\llbracket 0,1 \rrbracket \times T$ is defined in Lemma A.1, and where $H:[0,1] \times \operatorname{spt} T \rightarrow \mathcal{H}$ is the Lipschitz homotopy given by

$$
H(t, z):=t z+(1-t) F\left(\mathrm{p}^{\pi}(z)\right) .
$$

Then the image of $H$ is contained in $\left(\mathrm{p}^{\pi}\right)^{-1}\left(\mathrm{p}^{\pi}(\operatorname{spt} T)\right)$, and the claimed inclusion of the support of $V$ follows at once. From the interchangeability of $\partial$ and $H_{\sharp}$, (A.2), and $\partial T \equiv 0$ we moreover deduce that $V$ is normal with

$$
\partial V=H_{\sharp} \partial(\llbracket 0,1 \rrbracket \times T)=\left(H \circ \mathrm{i}_{1}\right)_{\sharp} T-\left(H \circ \mathrm{i}_{0}\right)_{\sharp} T=T-F_{\sharp}\left(\mathrm{p}^{\pi}\right)_{\sharp} T .
$$

To get the mass estimate in (A.5) we first notice $\operatorname{Lip}\left(H \circ \mathrm{i}_{t}\right) \leq 1+\operatorname{Lip}(f)$ for $t \in[0,1]$. Moreover, we have $\operatorname{Lip}(H(\cdot, z))=\left|z-F\left(\mathrm{p}^{\pi}(z)\right)\right|=\left|\mathrm{q}^{\pi}(z)-f\left(\mathrm{p}^{\pi}(z)\right)\right|$ for all $z \in \operatorname{spt} T$, which implies that $\left|\mathrm{D}_{t}\left(\psi_{j} \circ H\right) \circ \mathrm{i}_{t}\right| \leq\left|\mathrm{q}^{\pi}-f \circ \mathrm{p}^{\pi}\right|$ holds $\left(\mathscr{L}^{1} \otimes\|T\|\right)$-a. e. on $[0,1] \times \operatorname{spt} T$. Next we employ the definitions of pushforward, product, and mass together with the preceding observations in the following estimate ${ }^{13}$ for $(\varphi, \psi) \in \operatorname{Lip}_{\mathrm{b}}(\mathcal{H}) \times \operatorname{Lip}(\mathcal{H})^{n}$

$$
\begin{aligned}
\left|V\left(\varphi \bigwedge_{i=1}^{n} \mathrm{~d} \psi_{i}\right)\right| & =\left|(\llbracket 0,1 \rrbracket \times T)\left((\varphi \circ H) \bigwedge_{i=1}^{n} \mathrm{~d}\left(\psi_{i} \circ H\right)\right)\right| \\
& \leq \sum_{j=1}^{n} \int_{0}^{1}\left|T\left(\left(\varphi \circ H \circ \mathrm{i}_{t}\right)\left(\mathrm{D}_{t}\left(\psi_{j} \circ H\right) \circ \mathrm{i}_{t}\right) \bigwedge_{\substack{i=1 \\
i \neq j}}^{n}\left(\psi_{i} \circ H \circ \mathrm{i}_{t}\right)\right)\right| \mathrm{d} \mathscr{L}^{1}(t) \\
& \leq(1+\operatorname{Lip}(f))^{n-1} \sum_{j=1}^{n} \int_{0}^{1} \int_{\operatorname{spt} T}\left|\varphi \circ H \circ \mathrm{i}_{t}\right|\left|\mathrm{D}_{t}\left(\psi_{j} \circ H\right) \circ \mathrm{i}_{t}\right| \mathrm{d}\|T\| \mathrm{d} \mathscr{L}^{1}(t) \\
& \leq n(1+\operatorname{Lip}(f))^{n-1} \int_{0}^{1} \int_{\operatorname{spt} T}\left|\varphi \circ H \circ \mathrm{i}_{t}\right|\left|\mathrm{q}^{\pi}-f \circ \mathrm{p}^{\pi}\right| \mathrm{d}\|T\| \mathrm{d} \mathscr{L}^{1}(t) .
\end{aligned}
$$

As $\mathrm{q}^{\pi}-f \circ \mathrm{p}^{\pi}$ vanishes on Graph $f$ and is elsewhere controlled by (A.4), we finally get

$$
\left|V\left(\varphi \bigwedge_{i=1}^{n} \mathrm{~d} \psi_{i}\right)\right| \leq n K(1+\operatorname{Lip}(f))^{n-1} \int_{[0,1] \times(\operatorname{spt} T \backslash \operatorname{Graph} f)}|\varphi \circ H| \mathrm{d}\left(\mathscr{L}^{1} \otimes\|T\|\right) .
$$

We have thus shown

$$
\|V\| \leq n K(1+\operatorname{Lip}(f))^{n-1} H_{\sharp}\left(\left(\mathscr{L}^{1}\llcorner[0,1]) \otimes(\|T\|\llcorner(\operatorname{spt} T \backslash \operatorname{Graph} f))),\right.\right.
$$

and in particular the total mass estimate in (A.5) follows.

Finally, the remaining claim about conservation of (integer-)rectifiability follows from the fact that this property is preserved under both the product construction of Lemma A.1 and the pushforward operation.

\footnotetext{
${ }^{13}$ The compositions with $H$ and $H \circ i_{t}$ in this estimate are only defined on $[0,1] \times \operatorname{spt} T$ and $\operatorname{spt} T$, respectively. However, by the locality statement in [5, Theorem 3.5] this is sufficient to keep all relevant expressions well-defined.
} 


\section{Appendix B. Monotonicity Formula And DEnsity Results}

In this appendix, we denote by $C^{1}(\mathcal{H}, \mathcal{H})$ the space of Fréchet differentiable maps $\Phi: \mathcal{H} \rightarrow \mathcal{H}$ such that the derivative $\mathrm{D} \Phi$ is continuous from $\mathcal{H}$ to the space of linear maps in $\mathcal{H}$ endowed with the operator norm. We write Id for the identity map in $\mathcal{H}$, and we use the notation

$$
\operatorname{div}_{\vec{T}} \Phi(z):=\operatorname{trace}_{\vec{T}(z)}(\mathrm{D} \Phi(z))
$$

where the right-hand side is defined in (2.7).

Proposition B.1 (first variation). Let $\Omega \subset \mathcal{H}$ be open, let $T \in \mathbf{I}_{n}(\mathcal{H})$ be locally minimizing in $\Omega$, and consider $\Phi \in C^{1}(\mathcal{H}, \mathcal{H})$, with support at a positive distance from $\mathcal{H} \backslash \Omega$, such that $\mathrm{D} \Phi$ is bounded. Then we have

$$
\int_{\mathcal{H}} \operatorname{div}_{\vec{T}} \Phi \mathrm{d}\|T\|=0 .
$$

Proof. Given $L: \mathcal{H} \rightarrow \mathcal{H}$ linear and continuous, we denote $\mathrm{J}_{n}(L, \vec{T})$ the $n$-dimensional Jacobian in (2.5) with the $n$-plane $\pi$ given by $\vec{T}$. We write $\Psi_{\varepsilon}:=\operatorname{Id}+\varepsilon \Phi$ and notice that, for $\varepsilon$ small enough, $\Psi_{\varepsilon}$ is injective; in addition, the area formula and the local minimality of $T$ give

$$
\mathbf{M}(T) \leq \mathbf{M}\left(\left(\Psi_{\varepsilon}\right)_{\sharp} T\right)=\int_{\mathcal{H}} \mathrm{J}_{n}\left(\mathrm{D} \Psi_{\varepsilon}, \vec{T}\right) \mathrm{d}\|T\| .
$$

Using (2.6) (and the rule for the derivative of the square root), we can differentiate with respect to $\varepsilon$ to obtain (B.1).

Proof of (2.19). For $z \in \Omega$ and $0<\eta<\sigma<\operatorname{dist}(z, \partial \Omega)$ we will show that

$$
\frac{\|T\|\left(z+\mathbf{B}_{\eta}\right)}{\eta^{n}} \leq \frac{\|T\|\left(z+\mathbf{B}_{\sigma}\right)}{\sigma^{n}}
$$

We can assume, without loss of generality, $\|T\|\left(z+\partial \mathbf{B}_{\eta}\right)=\|T\|\left(z+\partial \mathbf{B}_{\sigma}\right)=0$. Under this extra assumption, an easy approximation argument shows that (B.1) still holds all for vector fields $\Phi$ of the form $\Phi(w)=\chi(|w-z|)(w-z)$, with $\chi:[0, \infty) \rightarrow[0, \infty)$ Lipschitz, with support in $[0, \operatorname{dist}(z, \partial \Omega))$, whose derivative $\chi^{\prime}(t)$ has at most jump discontinuities at $t=\eta$ and $t=\sigma$. Now we specifically insert in (B.1) the vector field

$$
\Phi(w):=\left[\min \left\{\eta^{-n},|w-z|^{-n}\right\}-\sigma^{-n}\right]_{+}(w-z),
$$

whose support is the closure of $z+\mathbf{B}_{\sigma}$. Then, the claim follows at once, when we calculate $\operatorname{div}_{\vec{T}} \Phi \equiv n\left[\eta^{-n}-\sigma^{-n}\right]$ on $z+\mathbf{B}_{\eta}$ and $\operatorname{div}_{\vec{T}} \Phi \geq-n \sigma^{-n}$ on $\left(z+\mathbf{B}_{\sigma}\right) \backslash\left(z+\mathbf{B}_{\eta}\right)$.

Proposition B.2. Consider $T \in \mathscr{I}_{n}(\mathcal{H})$. Then, for $\|T\|-a$. e. $z \in \mathcal{H}$ there hold

$$
\begin{gathered}
\lim _{r \searrow 0} r^{-n} \int_{z+\mathbf{B}_{r}}|\vec{T}-\vec{T}(z)|^{2} \mathrm{~d}\|T\|=0, \\
\lim _{r \searrow 0}\left\|T_{z, r}\right\|=\Theta(\|T\|, z) \mathscr{H}^{n} \mathrm{~L} \operatorname{Span} \vec{T}(z),
\end{gathered}
$$


where $I_{z, r}(w):=(w-z) / r, T_{z, r}:=\left(I_{z, r}\right)_{\sharp} T$ and the latter convergence is understood in duality with bounded continuous functions with bounded support.

Proof. Let us first reduce the proof to the case when $T=F_{\sharp} \llbracket \theta \rrbracket$ for some Lipschitz map $F: \mathbb{R}^{n} \rightarrow \mathcal{H}$ and $\theta \in \mathrm{L}^{1}\left(\mathbb{R}^{n}\right)$, with $\theta \in \mathbb{N} \cup\{0\} \mathscr{L}^{n}$-a. e. in $\mathbb{R}^{n}$ and $F$ bi-Lipschitz on $\theta^{-1}(\mathbb{N})$. Indeed, thanks to [5, Theorem 4.5], we can write any integer-rectifiable $T$ with finite mass as a $\mathbf{M}$-convergent series of currents $T_{i}$ of this form with pairwise disjoint measure-theoretic supports $S_{i}$. Since for any $i$ it holds

$$
\vec{T}=\vec{T}_{i} \quad \mathscr{H}^{n} \text {-a. e. on } S_{i}, \quad \Theta^{* n}\left(\sum_{j \neq i}\left\|T_{j}\right\|, z\right)=0 \quad \mathscr{H}^{n} \text {-a. e. on } S_{i}
$$

(for the second statement, see for instance $[20,2.10 .18(2)]$ ) we see that both (B.2) and (B.3) for $T$ at $\mathscr{H}^{n}$-a. e. point of $S_{i}$ follow from (B.2) and (B.3) for $S_{i}$.

So, let us assume $T=F_{\sharp} \llbracket \theta \rrbracket$ for some Lipschitz map $F: \mathbb{R}^{n} \rightarrow \mathcal{H}$ and some $\theta \in \mathrm{L}^{1}\left(\mathbb{R}^{n}\right)$ such that $\theta \in \mathbb{N} \cup\{0\}$ holds $\mathscr{L}^{n}$-a. e. in $\mathbb{R}^{n}$ and such that $F$ is bi-Lipschitz on $\theta^{-1}(\mathbb{N})$. We denote by $e_{1}, \ldots, e_{n}$ the canonical basis of $\mathbb{R}^{n}$. Using the area formula [6, Theorem 5.1], one can check by a straightforward computation that (B.2) holds at a point $z=F(x)$, with $\vec{T}(z)$ equal to the normalization of $\bigwedge_{i=1}^{n} \mathrm{D} F(x) e_{i}$, provided that we have $\Theta^{* n}(\|T\|, z)<\infty$ and that $x \in \theta^{-1}(\mathbb{N})$ satisfies

$$
\lim _{r \searrow 0} \frac{1}{\|\llbracket \theta \rrbracket\|\left(x+\mathbf{B}_{r}^{n}\right)} \int_{x+\mathbf{B}_{r}^{n}}|\mathrm{D} F-\mathrm{D} F(x)| \mathrm{d}\|\llbracket \theta \rrbracket\|=0 .
$$

Here, DF exists $\mathscr{L}^{n}$-a. e. on $\mathbb{R}^{n}$ by the Rademacher theorem [9, Theorem 5.11.1], (B.4) holds for $\|\llbracket \theta \rrbracket\|$-a. e. $x \in \mathbb{R}^{n}$, and all in all the preceding conditions are satisfied for $\|T\|$-a. e. $z \in \mathcal{H}$; thus, we arrive at the claim about (B.2).

In connection with (B.3), we consider $z=F(x)$ with $x \in \theta^{-1}(\mathbb{N})$ such that $F$ is classically Fréchet-differentiable at $x$ with $\operatorname{Span} \vec{T}(z)$ equal to the image of $\operatorname{D} F(x)$ and with

$$
\lim _{r \searrow 0} r^{-n} \int_{x+\mathbf{B}_{r}^{n}}\left[\left|\mathrm{~J}_{n}(\mathrm{D} F)-\mathrm{J}_{n}(\mathrm{D} F(x))\right|+|\theta-\theta(x)|\right] \mathrm{d} \mathscr{L}^{n}=0 .
$$

When we set $F_{r}(y):=(F(x+r y)-F(x)) / r$ and $\theta_{r}(y):=\theta(x+r y)$, then $F_{r}(y)$ converges to $\mathrm{D} F(x) y$ locally uniformly in $y \in \mathbb{R}^{n}$, on the level of the Jacobians $\mathrm{J}_{n}\left(\mathrm{D} F_{r}\right)$ converges to $\mathrm{J}_{n}(\mathrm{D} F(x))$ in $\mathrm{L}_{\text {loc }}^{1}\left(\mathbb{R}^{n}\right)$, and also $\theta_{r}$ converges to $\theta(x)$ in $\mathrm{L}_{\text {loc }}^{1}\left(\mathbb{R}^{n}\right)$. Furthermore, we have

$$
\left(I_{z, r}\right)_{\sharp} T=\left(F_{r}\right)_{\sharp} \llbracket \theta_{r} \rrbracket,
$$

and with the help of the preceding converges we conclude

$$
\begin{aligned}
\lim _{r \searrow 0} \int_{\mathcal{H}} \varphi \mathrm{d}\left\|T_{z, r}\right\| & =\lim _{r \searrow 0} \int_{\mathbb{R}^{n}} \varphi\left(F_{r}\right) \mathrm{J}_{n}\left(\mathrm{D} F_{r}\right) \theta_{r} \mathrm{~d} \mathscr{L}^{n} \\
& =\theta(x) \mathrm{J}_{n}(\mathrm{D} F(x)) \int_{\mathbb{R}^{n}} \varphi(\mathrm{D} F(x) y) \mathrm{d} \mathscr{L}^{n}(y)=\theta(x) \int_{\operatorname{Span} \vec{T}(z)} \varphi \mathrm{d} \mathscr{H}^{n}
\end{aligned}
$$


for every bounded continuous function $\varphi: \mathcal{H} \rightarrow \mathbb{R}$ with bounded support. As a side benefit of this convergence we also get $\Theta^{n}(\|T\|, z)=\lim _{r \searrow 0}\left\|T_{z, r}\right\|\left(\mathbf{B}_{1}\right) / \omega_{n}=$ $\theta(x)$. Similar as above, the assumed conditions on $z=F(x)$ hold true for $\mathscr{L}^{n}$-a. e. $x \in \theta^{-1}(\mathbb{N})$ and thus for $\|T\|$-a. e. $z \in \mathcal{H}$, so that we arrive at the claim regarding (B.3).

\section{REFERENCES}

1. F.J. Almgren, Almgren's big regularity paper. Q-valued functions minimizing Dirichlet's integral and the regularity of area-minimizing rectifiable currents up to codimension 2, World Scientific, Singapore, 2000.

2. F.J. Almgren, Existence and regularity almost everywhere of solutions to elliptic variational problems among surfaces of varying topological type and singularity structure, Ann. Math. 87 (1968), 321-391.

3. L. Ambrosio, Metric space valued functions of bounded variation, Ann. Sc. Norm. Super. Pisa, Cl. Sci., IV. Ser. 17 (1990), 439-478.

4. L. Ambrosio, N. Fusco, and D. Pallara, Functions of bounded variation and free discontinuity problems, Oxford University Press, Oxford, 2000.

5. L. Ambrosio and B. Kirchheim, Currents in metric spaces, Acta Math. 185 (2000), 1-80.

6. L. Ambrosio and B. Kirchheim, Rectifiable sets in metric and Banach spaces, Math. Ann. 318 (2000), 527-555.

7. L. Ambrosio and T. Schmidt, Compactness results for normal currents and the Plateau problem in dual Banach spaces, Proc. Lond. Math. Soc. (3), to appear (2012), 22 pages.

8. G. Anzellotti and M. Giaquinta, Convex functionals and partial regularity, Arch. Ration. Mech. Anal. 102 (1988), 243-272.

9. V.I. Bogachev, Gaussian measures, American Mathematical Society, Providence, 1998.

10. E. Bombieri, Regularity theory for almost minimal currents, Arch. Ration. Mech. Anal. 78 (1982), 99-130.

11. A.P. Calderón and A. Zygmund, On the existence of certain singular integrals, Acta Math. 88 (1952), 85-139.

12. E. De Giorgi, Frontiere orientate di misura minima, Seminario di Matematica, Sc. Norm. Super. Pisa, 1960-1961.

13. C. De Lellis and E. Spadaro, Center manifold: A case study, Discrete Contin. Dyn. Syst. 31 (2011), 1249-1272.

14. C. De Lellis and E. Spadaro, Q-valued functions revisited, Mem. Am. Math. Soc. 211 (2011), no. 991,79 pages.

15. C. De Lellis and E. Spadaro, Higher integrability and approximation of minimal currents, Preprint (2012), 39 pages.

16. T. De Pauw and R. Hardt, Rectifiable and flat $G$ chains in a metric space, Am. J. Math. 134 (2012), 1-69.

17. F. Duzaar and K. Steffen, Optimal interior and boundary regularity for almost minimizers to elliptic variational integrals, J. Reine Angew. Math. 546 (2002), 73-138.

18. L.C. Evans, Partial Differential Equations, American Mathematical Society, Providence, 1998.

19. L.C. Evans and R.F. Gariepy, Measure theory and fine properties of functions, CRC Press, Boca Raton, 1992.

20. H. Federer, Geometric Measure Theory, Springer, Berlin, 1969.

21. H. Federer, The singular sets of area minimizing rectifiable currents with codimension one and of area minimizing flat chains modulo two with arbitrary codimension, Bull. Am. Math. Soc. 76 (1970), 767-771. 
22. D. Gilbarg and N.S. Trudinger, Elliptic Partial Differential Equations of Second Order. 2nd ed., Springer, Berlin, 1983.

23. E. Giusti, Minimal Surfaces and Functions of Bounded Variation, Birkhäuser, Basel, 1984.

24. E. Giusti, Direct Methods in the Calculus of Variations, World Scientific Publishing, Singapore, 2003.

25. R.L. Jerrard, A new proof of the rectifiable slices theorem, Ann. Sc. Norm. Super. Pisa, Cl. Sci. (5) 1 (2002), 905-924.

26. R.L. Jerrard and H.M. Soner, Rectifiability of the distributional Jacobian for a class of functions, C. R. Acad. Sci., Paris, Sér. I, Math. 329 (1999), 683-688.

27. R.L. Jerrard and H.M. Soner, Functions of bounded higher variation, Indiana Univ. Math. J. 51 (2002), 645-677.

28. B. Kirchheim, Rectifiable metric spaces: Local structure and regularity of the Hausdorff measure, Proc. Am. Math. Soc. 121 (1994), 113-123.

29. U. Lang, Local currents in metric spaces, J. Geom. Anal. 21 (2011), 683-742.

30. E.R. Reifenberg, On the analyticity of minimal surfaces, Ann. Math. (2) 80 (1964), 15-21.

31. T. Schmidt, Partial regularity for degenerate variational problems and image restoration models in BV, Indiana Univ. Math. J., to appear (2013), 54 pages.

32. R. Schoen and L. Simon, A new proof of the regularity theorem for rectifiable currents which minimize parametric elliptic functionals, Indiana Univ. Math. J. 31 (1982), 415-434.

33. J. Simons, Minimal varieties in riemannian manifolds, Ann. Math. (2) 88 (1968), 62-105.

34. I. Tamanini, Regularity results for almost minimal oriented hypersurfaces in $\mathbb{R}^{n}$, Quaderni del Dipartimento di Matematica dell' Università di Lecce (1984), no. 1, 92 pages.

35. F.A. Valentine, A Lipschitz condition preserving extension for a vector function, Am. J. Math. 67 (1945), 83-93.

36. S. Wenger, Isoperimetric inequalities of Euclidean type in metric spaces, Geom. Funct. Anal. 15 (2005), 534-554.

37. S. Wenger, Plateau's problem for integral currents in locally non-compact metric spaces, Preprint (2012), 10 pages.

38. B. White, Rectifiability of flat chains, Ann. Math. (2) 150 (1999), 165-184.

39. B. White, The deformation theorem for flat chains, Acta Math. 183 (1999), 255-271.

(L. Ambrosio and T. Schmidt) SNS Pisa, Piazza Dei Cavalieri 7, 56126 Pisa, Italy

E-mail address: luigi.ambrosio@sns.it

E-mail address: thomas.schmidt@sns.it

(C. De Lellis and T. Schmidt) Institut Für Mathematik, Universität Zürich, WinterthurerSTRASSE 190, 8057 ZURICH, SWITZERLAND

E-mail address: camillo.delellis@math.uzh.ch

E-mail address: thomas.schmidt@math.uzh.ch 